\title{
Robust shape and topology optimization of nanophotonic devices using the level set method
}

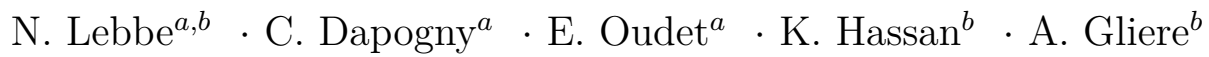 \\ ${ }^{a}$ Univ. Grenoble Alpes, CNRS, Grenoble INP ${ }^{1}$, LJK, 38000 Grenoble, France \\ ${ }^{b}$ Univ. Grenoble Alpes, CEA, LETI, F38000 Grenoble, France
}

\begin{abstract}
This article deals with the optimal design of the individual nanophotonic components of photonic integrated circuits. In the mathematical setting of the three-dimensional, time-harmonic Maxwell equations, we propose a shape and topology optimization algorithm combining Hadamard's boundary variation method with a level set representation of shapes and their evolution. A particular attention is devoted to the robustness of the optimized devices with respect to small uncertainties over the physical or geometrical data of the problem. In this respect, we rely on a simple multi-objective formulation to deal with the two main sources of uncertainties plaguing nanophotonic devices, namely uncertainties over the incoming wavelength, and geometric uncertainties entailed by the lithography and etching fabrication process. Several numerical examples are presented and discussed to assess the efficiency of our methodology.
\end{abstract}

Keywords shape optimization $\cdot$ level-set method $\cdot$ nanophotonics $\cdot$ robustness $\cdot$ Maxwell equations

Mathematics Subject Classification (2010) 49Q10 $65 \mathrm{~K} 10 \cdot 74 \mathrm{P} 20$

\section{Contents}

1 Introduction $\mathbf{2}$

2 The Maxwell equations for nanophotonic devices 4

2.1 A brief presentation of nanophotonic devices . . . . . . . . . . . . . . . . . . . 4

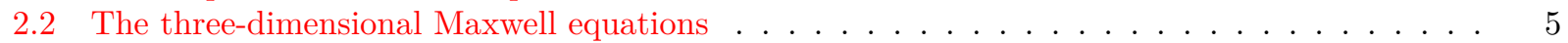

2.3 Propagation modes in waveguides . . . . . . . . . . . . . . . . . . . 6

2.4 Boundary conditions . . . . . . . . . . . . . . . . . . . . . . . . . 8

2.4.1 Injection of a particular mode at the entrance port $\Gamma_{\text {in }} \ldots . . . . . . . . . .99$

2.4.2 Approximation of the decay conditions at infinity using a Perfectly Matched Layer . . 10

2.5 The complete variational framework . . . . . . . . . . . . . . . . . . 11

3 Shape optimization of nanophotonic devices 11

3.1 Presentation of the shape optimization problem . . . . . . . . . . . . . . . 11

3.2 Differentiation with respect to the domain using Hadamard's boundary variation method . . 12

3.3 Shape derivatives of the considered objective functional . . . . . . . . . . . . . . . 13

3.3.1 The 'exact' shape derivative of $J(\Omega) \ldots \ldots \ldots \ldots \ldots$

3.3.2 Numerical approximation of the shape derivative . . . . . . . . . . . . . . . 14

\footnotetext{
${ }^{1}$ Institute of Engineering Univ. Grenoble Alpes
} 
4 Robustness in the optimization of nanophotonic devices

4.1 A general abstract setting . . . . . . . . . . . . . . . . . . . . . . 15

4.2 Robustness with respect to the wavelength . . . . . . . . . . . . . . . . 16

4.3 Robustness with respect to geometric uncertainties . . . . . . . . . . . . . . . . . 17

4.3.1 Robustness with respect to uncertainties caused by the etching process: an approach using dilation and erosion . . . . . . . . . . . . . . . . . .

4.3.2 Robust shape optimization with respect to defects caused by the lithography process: a description using Gaussian kernels . . . . . . . . . . . . . . . . 18

5 Numerical representation of shapes and general algorithm $\mathbf{2 0}$

5.1 Level set representation . . . . . . . . . . . . . . . . . . . . . . 20

5.2 Implementation and algorithm . . . . . . . . . . . . . . . . . . . . 21

6 Numerical examples $\mathbf{2 1}$

6.1 Optimization examples involving a single objective function . . . . . . . . . . . . . . . . 22

6.1.1 Optimization of the shape of a crossing device . . . . . . . . . . . . . . . 22

6.1.2 Optimization of the shape of a mode converter . . . . . . . . . . . . . . . 23

6.1.3 Shape optimization of a power divider . . . . . . . . . . . . . . . . . . . . 24

6.1.4 Optimization of the shape of a polarization converter . . . . . . . . . . . . . . . . . . . . . . . . . . .

6.2 Optimization examples involving multiple objective functions . . . . . . . . . . . . . . . . . . . . . . . . . .

6.2.1 Optimization of the shape of a duplexer . . . . . . . . . . . . . . . . . . . . . . . . . . . .

6.2.2 Optimization of the shape of a power divider with three output waveguides . . . . . 28

6.3 Robust shape optimization of a duplexer with respect to uncertainties on the incoming wavelength 29

6.4 Robust shape optimization of nanophotonic devices with respect to geometric uncertainties . 30

6.4.1 Robust shape optimization of a power divider with respect to uncertainties caused by

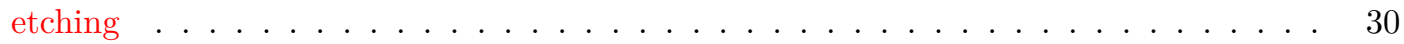

6.4.2 Robust optimization of a mode converter with respect to uncertainties caused by etching 31

6.4.3 Robust optimization of a duplexer with respect to uncertainties caused by etching . . 32

6.4.4 Robust optimization of a mode converter with respect to uncertainties caused by lithog-

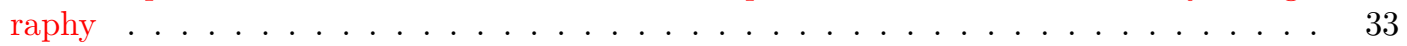

Appendices $\quad 36$

A Sketch of proof of Theorem 3.1 36

B Proof of Proposition 4.1 39

\section{Introduction}

Nanophotonic devices are components used to manipulate light, considered as an electromagnetic field, at the nanometric scale. They are tailored to accomplish specific tasks such as guiding an incident wave with negligible loss, splitting it into several output ports, converting a mode from an incoming waveguide into another mode of an outgoing waveguide, etc. Nanophotonic devices are the basic components of the photonic integrated circuits (PICs) used, for instance, in fiber optic communications, microscopy, biosensing and even in the prospective research about photonic computing (see [27] for an overview).

Their non trivial properties are generally obtained as the result of a clever repartition of "core" and "cladding" materials, in a context where the difficult underlying physics leaves very little room for intuition; this raises the need for advanced methods stemming from numerical simulation and mathematical programming for the design of nanophotonic devices. While the development of integrated optical functionalities has historically relied on a dedicated opto-geometrical optimization of each functionality with respect to a restricted number of parameters, modern shape and topology optimization algorithms have naturally emerged as a unified solution. These techniques have indeed been applied in a wide variety of situations in the photonic context for about ten to twenty years [46, 11], even though the practical realization of such components at the micrometric scale has been achieved only very recently [58, 29]. Among the structures considered in the literature, let us mention photonic crystals which are designed to allow the existence of band gaps (i.e. ranges of optical frequencies at which waves cannot propagate) [49, 70], or grating couplers, whose shape is 
optimized with respect to the light power transmitted from an optical fiber into a waveguide [67] (see also $[29,45]$ for further examples).

In realistic applications, the physical description of nanophotonic devices involves morphological and environmental parameters whose knowledge is uncertain. For instance, the optical indices of the constituent materials depend on the ambient temperature which may be fluctuating in time. Likewise, the incoming wavelength into the device may be subject to variations caused by fabrication issues or by experimental uncertainties (over e.g. the ambient temperature, or the accuracy of the laser used for light injection). One last - and critical - source of uncertainty plaguing industrial nanophotonic devices is related to the fabrication process used for their assembly, an issue which deserves a brief comment. Even though a mature CMOS (Complementary metal oxide semi-conductor) monolithic integration is employed to realize optical chips, the high volume of device produced on a single wafer (semi-conductor plate) is a well-known cause of manufacturing dispersion. The main variations and uncertainties plaguing nanophotonic devices are induced by the lithography and etching stages of the fabrication process, that impact directly their morphology. In a nutshell, lithography uses a high energy optical (deep UV) or electron-beam flux to pattern a thin resistive layer with the desired shapes, which are subsequently transferred to the semiconductor underneath with a selective etching [37]. Fluctuations of the energy during the lithography process, or on the resistive quality of the layer will modify the shape before it is imprinted on the semiconductor, while the etching process itself might shrink or enlarge the patterns, depending on multiple factors, such as the density of the pattern, minimum feature sizes, etc. In any event, whatever the uncertainties at play, the performance of the predicted shapes by optimal design methods strongly depends on the particular set of physical data; hence, their optimal character may end up completely ruined by even small, unpredicted variations of these. This raises the need to incorporate into these algorithms a degree of robustness with respect to small variations of these data.

Most contributions from the literature considering the optimal design of nanophotonic devices rely on density methods $[39,25,45]$. This popular paradigm, originally introduced in the context of structural mechanics as a heuristic approximation of mathematical homogenization (and known in this context as the SIMP method [9]) amounts to trade the conventional "black-and-white" representation of a design - via its characteristic function, taking only the values 0 or 1 outside and inside the latter - for a so-called "grayscale" density function, with values in the whole interval $[0,1]$. This considerably simplifies the optimization process, but raises the need for an approximate modelling of the physical equations (which have to be expressed in terms of the density function), as well as issues about the interpretation of the resulting design, and notably of its "grayscale" regions, where the density function takes intermediate values in $[0,1]$. On the other hand, more "geometric" methods, featuring a clear representation of the boundary of the optimized shape, have been considered, relying for instance on the concept of shape or topological derivatives [43, 46, 7, 60]): the shape is iteratively modified by either deforming its contour or by inserting small holes inside it. Alternatively, let us mention the use of costly genetic algorithms in the nanophotonic context [64, 72].

Since the first achievements of shape and topology optimization studies in nanophotonics, the robustness of the produced devices with respect to small variations of the physical parameters has been acknowledged as a key issue in their realistic utilization [45, Section 4.5]. However, to the best of our knowledge, very few (and recent) methods have been devised hitherto so as to directly incorporate uncertainty awareness into the optimization process. In this direction, robustness with respect to the incoming wavelength has been taken into account by relying on various simulations at multiple wavelengths [58]; robustness with respect to manufacturing uncertainties (entailed for instance by the lithography and etching process) have been studied using the SIMP methodology by involving the dilated and eroded versions of a given design; see [65, 70], or [24] for a probabilistic counterpart to these studies. Let us point out the contribution [59], where the authors impose fabrication constraints by removing at each iteration of the optimization process the patterns that would not be manufactured properly.

In the present article, we propose a multi-objective algorithm for the optimization of nanophotonic devices in a way which is robust to uncertainties over parameters such as the incoming wavelength or the geometry of the optimized shape itself. To achieve this, we rely on Hadamard's method for calculating the sensitivity of shapes with respect to small variations of the boundary of the design, and we use it in a numerical framework based on the level set method for shape and topology optimization. Doing so enables a precise geometric representation of shapes, which makes it possible to model and consider uncertainties over all kinds of parameters, including geometric ones, in a fashion close to their mathematical modeling.

The remainder of this article is organized as follows. Section 2 is a short and formal introduction to the physics governing nanophotonic devices, and to the associated mathematical setting. Section 3 then 
describes our shape optimization framework for nanophotonic devices: the considered problems are precisely formulated, and basic notions about shape derivatives are outlined in this context. Section 4 deals with the modeling and the mathematical formulation of robustness with respect to uncertainties over the wavelength of the incident wave, or with respect to the imperfections brought about by the lithography and etching manufacturing processes. Section 5 describes the numerical method used to represent shapes and their deformations, and the shape optimization algorithm derived from the previous considerations is sketched. Finally, Section 6 discusses multiple numerical examples in three-dimensional situations reflecting the various aspects of the article. This article is supplemented with two appendices, devoted to the proof of some mathematically technical results, including a formal outline of the calculation of the shape derivatives involved in our investigations.

\section{The Maxwell equations for nanophotonic devices}

In this section, we present the physical description of the nanophotonic devices considered in this article and we briefly outline the needed ingredients for their study, without entering too much into the underlying mathematical theory.

\subsection{A brief presentation of nanophotonic devices}

The nanophotonic devices of interest pertain to the field of silicon photonics, which features photonic integrated circuits composed of a base wafer, on which semiconductor components are patterned by means of the CMOS compatible microfabrication techniques.

Silicon On Insulator (SOI) base wafers have recently aroused a tremendous enthusiasm among the integrated optics community for their relatively simple and cheap production, and for their high efficiency in terms of energy confinement [42, Chap. 13]. Accordingly, the nanophotonic devices considered in this article feature a core of silicon $(\mathrm{Si})$ patterned on top of a silica $\left(\mathrm{SiO}_{2}\right)$ substrate. Moreover, in order to improve the contrast between optical indices, and thus the light guiding characteristics, the silicon pattern is surrounded by a cladding of air. Notice that, depending on the application (and on the considered range of wavelength for the incoming light), different core/cladding couples of materials can be addressed, such as for instance $\mathrm{Si} / \mathrm{SiO}_{2}$ or $\mathrm{Ge} / \mathrm{SiGe}$, by relying on the mathematical and numerical frameworks discuss in this article.

The peculiar, targeted properties of such nanophotonic devices are achieved by acting on the geometry of the repartition of core and cladding materials within a given design space $\mathcal{D}_{\text {opt }}$, shaped as a box, as depicted in Fig. 1a. The latter is connected to one or several waveguides conveying the incoming and outgoing electromagnetic waves, and the resulting configuration is placed on a lower layer $\mathcal{D}_{\text {subs }}$ (also referred to as the substrate), made of insulator - typically silica $\mathrm{SiO}_{2}$; see Fig. 1b. The refractive index $n_{\text {core }}$ of the core material (silicon) is much larger than those $n_{\text {clad }}$ and $n_{\text {subs }}$ of the cladding (air) and substrate (silica), this allows for a strong confinement of light inside the core phase.

Let us now make more precise the mathematical and numerical setting of this article, which is illustrated in Fig. 1. We only rely on the three-dimensional physical description of our nanophotonic devices; even though approximate two-dimensional approximations exist (see for instance [13, Chapter 5], about the socalled "effective index method"), our experience suggests that they are not accurate enough for our purpose as was also pointed out in [25]. We define

- The box $\mathcal{D}=\left[-w_{x}, w_{x}\right] \times\left[-w_{y}, w_{y}\right] \times\left[-w_{z}, w_{z}\right] \subset \mathbb{R}^{3}$ is the total computational domain, accounting for the whole three-dimensional space - at least the region where it is relevant to consider the electromagnetic fields surrounding the device.

- $\mathcal{D}_{\text {opt }} \subset \mathcal{D}$ is the fixed design domain; it is typically a box with small thickness $h$ in the $y$ direction, containing all possible shapes $\Omega$.

- The considered shapes $\Omega \subset \mathcal{D}_{\text {opt }}$ are Lipschitz domains accounting for the region of $\mathcal{D}_{\text {opt }}$ filled with core material, and so the complementary phase $\mathcal{D}_{\text {opt }} \backslash \bar{\Omega}$ is occupied by cladding. Due to their fabrication by etching, we will look for shapes $\Omega$ which are invariant in the $y$ direction, and denote their $2 \mathrm{~d}$ section in the $(x, z)$ plane by $\widehat{\Omega}$ :

$$
\Omega=\left\{\mathbf{x}=(x, y, z) \in \mathcal{D}_{\mathrm{opt}}, \quad(x, z) \in \widehat{\Omega}, y \in(0, h)\right\} .
$$


- $\mathcal{D}_{\text {wg }} \subset \mathcal{D}$ is the region occupied by the input and output waveguides.

- $\mathcal{D}_{\text {subs }} \subset \mathcal{D}$ is the layer supporting $\mathcal{D}_{\text {opt }}$, occupied by the substrate.

- $\mathcal{D}_{\mathrm{PML}} \subset \mathcal{D}$ is a "Perfectly Matched Layer", a region of $\mathcal{D}$ filled with absorbing material aimed at imposing the correct behavior of the electromagnetic fields at infinity; see Section 2.4.2.

- $\Gamma_{\text {in }} \subset \partial \mathcal{D}$ is a region of the boundary of $\mathcal{D}$ accounting for the entrance of a waveguide into the device; see Section 2.4.1.

- $\Gamma_{\text {obj }} \Subset \mathcal{D}$ is an internal surface in $\mathcal{D}$ used for the computation of the optimization objective; see Section 3.1.
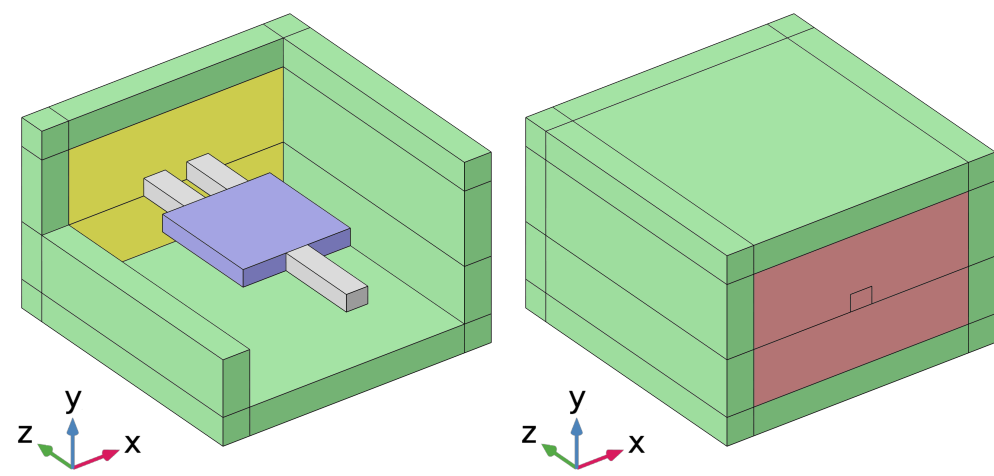

(a) The different regions of the computational domain $\mathcal{D}$ : $P M L$ domain $\mathcal{D}_{\mathrm{PML}}$ in green, design domain $\mathcal{D}_{\mathrm{opt}}$ in blue, input and output waveguides $\mathcal{D}_{\mathrm{wg}}$ in grey, input surface $\Gamma_{\text {in }}$ in red and objective surface $\Gamma_{\text {obj }}$ in yellow.

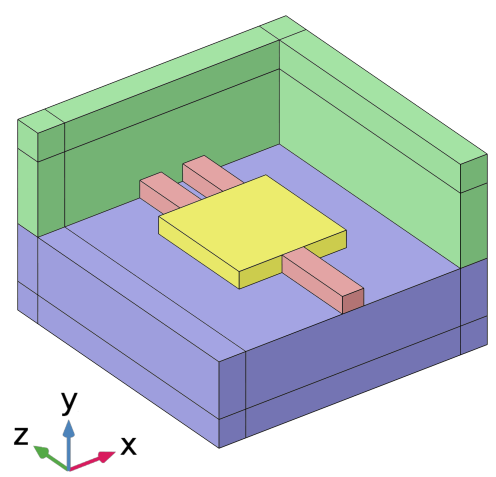

(b) Values of the refractive index $n$ inside $\mathcal{D}$ : $n=n_{\text {core }}$ or $n_{\text {clad }}$ in the yellow design domain, $n=n_{\text {ncore }}$ in the input and output waveguides $\mathcal{D}_{\mathrm{wg}}, n=n_{\mathrm{subs}}$ in the blue substrate layer, and $n=n_{\text {clad }}$ in the remaining areas.

Figure 1 - Schematic representation of the physical setting of Section 2.1.

\subsection{The three-dimensional Maxwell equations}

In the situation of Section 2.1, the physical behavior of the electric and magnetic fields surrounding the considered nanophotonic devices is described by the classical Maxwell equations; see for instance [40, Sections $1.2 \& 1.4],[32$, Section 7.3.3] or [53, Chapter 1].

Our study takes place at fixed optical frequency $\omega$ (whence the wavenumber $k=\omega / c$ and the wavelength $\lambda=2 \pi c / \omega$ are inferred from the velocity of light $c=2.99792458 \times 10^{8} \mathrm{~m} \cdot \mathrm{s}^{-1}$ ), and a time dependence of the form $e^{-i \omega t}$ is assumed throughout the article. The considered materials (core, cladding and substrate) are all linear, isotropic, non dispersive and non absorbing, so that the time-harmonic version of the Maxwell equations reads:

$$
\begin{aligned}
& \nabla \times \mathbf{E}=i \omega \mu \mathbf{H}, \quad \text { Maxwell-Faraday } \\
& \nabla \cdot(\varepsilon \mathbf{E})=\quad 0, \quad \text { Maxwell-Gauss } \\
& \nabla \times \mathbf{H}=-i \omega \varepsilon \mathbf{E}, \quad \text { Maxwell-Ampère } \\
& \nabla \cdot(\mu \mathbf{H})=0, \quad \text { Maxwell-Thomson }
\end{aligned}
$$

where the curl $\nabla \times \mathbf{u}$ and divergence $\nabla \cdot \mathbf{u}$ of a three-dimensional vector field $\mathbf{u}=\left(u_{x}, u_{y}, u_{z}\right)$ are defined by:

$$
\nabla \times \mathbf{u}=\left(\frac{\partial u_{z}}{\partial y}-\frac{\partial u_{y}}{\partial z}, \frac{\partial u_{x}}{\partial z}-\frac{\partial u_{z}}{\partial x}, \frac{\partial u_{y}}{\partial x}-\frac{\partial u_{x}}{\partial y}\right)^{T}, \text { and } \nabla \cdot \mathbf{u}=\frac{\partial u_{x}}{\partial x}+\frac{\partial u_{y}}{\partial y}+\frac{\partial u_{z}}{\partial z} .
$$

In $(2.2)$ to $(2.5), \varepsilon$ is the dielectric permittivity, and $\mu$ is the magnetic permeability. 
As is well-known, the electric and magnetic fields $\mathbf{E}$ and $\mathbf{H}$ may be equivalently characterized as the solutions to two decoupled second-order wave-like equations, instead of the first-order system (2.2) to (2.5); indeed, applying the $\nabla \times$ operator to (2.2) and using (2.4) yields:

$$
\nabla \times\left(\frac{1}{\mu} \nabla \times \mathbf{E}\right)-\omega^{2} \varepsilon \mathbf{E}=0 .
$$

In the same way, we obtain for the magnetic field:

$$
\nabla \times\left(\frac{1}{\varepsilon} \nabla \times \mathbf{H}\right)-\omega^{2} \mu \mathbf{H}=0 .
$$

Moreover, from the knowledge of $\mathbf{E}$ (resp. $\mathbf{H}$ ), the values of $\mathbf{H}$ (resp. $\mathbf{E}$ ) are easily recovered owing to (2.2) (resp. (2.4)). For this reason, in the following, we rely on the single equation (2.6) to characterize the behavior of the electromagnetic fields.

In our applications, the permittivity $\varepsilon$ equals a positive real constant inside each phase, characterizing the physical properties of its constituent material, whereas the permeability $\mu$ is constant over the whole space: $\mu=\mu_{0}$ where $\mu_{0}=4 \pi 10^{-7}$ is the vacuum permeability. Then introducing the refractive index $n^{2}:=\varepsilon / \varepsilon_{0}$, whose definition involves the vacuum permittivity $\varepsilon_{0}=1 /\left(\mu_{0} c^{2}\right)$, (2.6) and (2.7) simply rewrite:

$$
\nabla \times \nabla \times \mathbf{E}-k^{2} n^{2} \mathbf{E}=0
$$

and

$$
\nabla \times\left(\frac{1}{n^{2}} \nabla \times \mathbf{H}\right)-k^{2} \mathbf{H}=0 .
$$

In the physical situation of Section 2.1, the optical index $n$ featured in (2.8) and (2.9) depends on the geometry $\Omega$ of the repartition of core and cladding materials within the design domain $\mathcal{D}_{\text {opt }}$. We shall denote by $n_{\Omega}$ (resp. $\mathbf{E}_{\Omega}, \mathbf{H}_{\Omega}$ ) the refractive index (resp. the corresponding electric and magnetic fields) within $\mathcal{D}$ when the core material occupies the region $\Omega \subset \mathcal{D}_{\text {opt }}$; more precisely:

$$
\forall \mathbf{x} \in \mathcal{D}, n_{\Omega}(\mathbf{x})= \begin{cases}n_{\text {core }} & \text { if } \mathbf{x} \in \Omega \cup \mathcal{D}_{\text {wg }}, \\ n_{\text {subs }} & \text { if } \mathbf{x} \in \mathcal{D}_{\text {subs }}, \\ n_{\text {clad }} & \text { if } \mathbf{x} \in \mathcal{D} \backslash\left(\bar{\Omega} \cup \overline{\mathcal{D}_{\text {wg }}} \cup \overline{\mathcal{D}_{\text {subs }}}\right) .\end{cases}
$$

In addition, the mathematical characterization of the considered physical setting demands that (2.8) and (2.9) be complemented with adequate boundary conditions describing the behavior of $\mathbf{E}$ and $\mathbf{H}$ at the entrance port $\Gamma_{\mathrm{in}}$, and its decay at infinity. The mathematical formulation of these boundary conditions is described in Section 2.4.

\subsection{Propagation modes in waveguides}

Propagation modes describe the fundamental structure of electromagnetic waves propagating in an infinite waveguide. As such, they play a central role in the boundary conditions accounting for injection of light at the entrance ports of nanophotonic devices (see Section 2.4), as well as in the expression of our optimization objectives in Section 3.1 .

The waveguides of interest for our purposes (see Fig. 2) are components of the considered photonic integrated circuit. Such waveguides are open, i.e. their transverse section is unbounded; see for instance [23, 31, 66] for basics about their study. Let us consider one such waveguide, whose longitudinal direction (i.e. the direction in which it is infinite) is $\hat{\mathbf{z}}$, and whose transverse section is denoted by $\mathcal{S}$. In this context, the refractive index $n_{\mathrm{wg}}$ is invariant in the $z$ direction $n_{\mathrm{wg}}(\mathbf{x}) \equiv n_{\mathrm{wg}}(x, y)$.

The electric and magnetic fields $\mathbf{E}(\mathbf{x})$ and $\mathbf{H}(\mathbf{x})$ inside the waveguide, solution to (2.2) to (2.5), may be decomposed as follows:

$$
\mathbf{E}(\mathbf{x})=\sum_{j=1}^{N} a_{j} \mathbf{E}_{j}(\mathbf{x})+\sum_{j=1}^{N} a_{-j} \mathbf{E}_{-j}(\mathbf{x})+\mathbf{E}_{\mathrm{rad}}(\mathbf{x}), \text { and } \mathbf{H}(\mathbf{x})=\sum_{j=1}^{N} a_{j} \mathbf{H}_{j}(\mathbf{x})+\sum_{j=1}^{N} a_{-j} \mathbf{H}_{-j}(\mathbf{x})+\mathbf{H}_{\mathrm{rad}}(\mathbf{x})
$$

where the coefficients $a_{j} \in \mathbb{C}$ appearing in both expressions are identical. The expression (2.11) features two kinds of modes: 


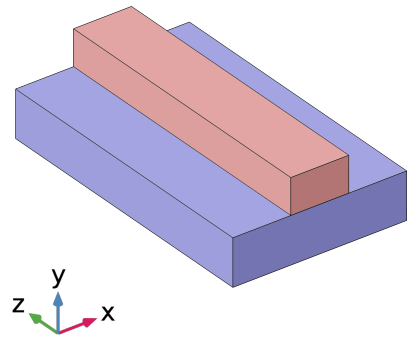

(a) A photonic waveguide

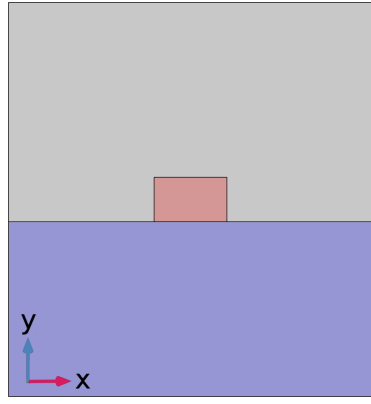

(b) Repartition of the refractive index $n$ inside $\mathcal{S}$ : $n=$ $n_{\text {core }}$ in the red core layer, $n=n_{\text {subs }}$ in the blue substrate layer, and $n=n_{\text {clad }}$ in the remaining areas,

Figure 2 - Schematic representation of the photonic waveguides considered in Section 2.3.

- A finite number of bound or guided modes $\left(\mathbf{E}_{j}, \mathbf{H}_{j}\right), j=1, \ldots, N$, whose energy is concentrated in the vicinity of the core region of the waveguide;

- An infinite collection of radiating modes gathered in the fields $\left(\mathbf{E}_{\mathrm{rad}}, \mathbf{H}_{\mathrm{rad}}\right)$, whose energy is unbounded in the transverse section $\mathcal{S}$ of the waveguide.

In the decomposition (2.11), the guided modes arise under the separated form

$$
\mathbf{E}_{j}(\mathbf{x})=\mathbf{e}_{j}(x, y) e^{i \beta_{j} z} \text {, and } \mathbf{H}_{j}(\mathbf{x})=\mathbf{h}_{j}(x, y) e^{i \beta_{j} z}, j=-N, \ldots,-1 \text {, and } 1, \ldots, N,
$$

where the $\beta_{j}$ are real constants. Let us introduce the longitudinal and transverse components of $\mathbf{e}_{j}, e_{z j}$ and $\mathbf{e}_{\tau j}:=e_{x j} \widehat{\mathbf{x}}+e_{y j} \widehat{\mathbf{y}}$ respectively (and likewise for $\mathbf{h}_{j}$ ), so that:

$$
\mathbf{e}_{j}=\mathbf{e}_{\tau j}+e_{z j} \hat{\mathbf{z}} \text {, and } \mathbf{h}_{j}=\mathbf{h}_{\tau j}+h_{z j} \hat{\mathbf{z}} \text {. }
$$

By convention, the modes corresponding to the indices $j=1, \ldots, N$ are propagating in the forward sense of the waveguide, i.e. $\beta_{j}>0$, and those attached to $j=-N, \ldots,-1$ are propagating backward, that is $\beta_{j}<0$. Both sets of modes are related by:

$$
\beta_{-j}=-\beta_{j}, \mathbf{e}_{-j}=\mathbf{e}_{\tau j}-e_{z j} \hat{\mathbf{z}}, \text { and } \mathbf{h}_{-j}=-\mathbf{h}_{\tau j}+h_{z j} \hat{\mathbf{z}}, j=1, \ldots, N .
$$

Let us now focus on the forward propagating modes; the values $\beta_{j}$ and the corresponding longitudinal components $\left(e_{j z}, h_{j z}\right)$ of the electric and magnetic fields are found as the (normalized) eigenelements $\left(\beta, e_{z}, h_{z}\right)$ of the following $2 \mathrm{~d}$ differential operator, acting on the transverse section $\mathcal{S}$ :

$\left\{\begin{array}{l}\Delta_{\tau} e_{z}+\left(k^{2} n_{\mathrm{wg}}^{2}-\beta^{2}\right) e_{z}-\frac{\beta^{2}}{k^{2} n_{\mathrm{wg}}^{2}-\beta^{2}} \nabla_{\tau} e_{z} \cdot \nabla_{\tau}\left(\log \left(n_{\mathrm{wg}}^{2}\right)\right)=-\left(\frac{\mu_{0}}{\varepsilon_{0}}\right)^{1 / 2} \frac{k \beta}{k^{2} n_{\mathrm{wg}}^{2}-\beta^{2}}\left(\nabla_{\tau} h_{z} \times \nabla_{\tau}\left(\log \left(n_{\mathrm{wg}}^{2}\right)\right) \cdot \hat{\mathbf{z}},\right. \\ \Delta_{\tau} h_{z}+\left(k^{2} n_{\mathrm{wg}}^{2}-\beta^{2}\right) h_{z}-\frac{n_{\mathrm{wg}}^{2} k^{2}}{k^{2} n_{\mathrm{wg}}^{2}-\beta^{2}} \nabla_{\tau} e_{z} \cdot \nabla_{\tau}\left(\log \left(n_{\mathrm{wg}}^{2}\right)\right)=-\left(\frac{\mu_{0}}{\varepsilon_{0}}\right)^{1 / 2} \frac{k n_{\mathrm{wg}}^{2} \beta}{k^{2} n_{\mathrm{wg}}-\beta^{2}}\left(\nabla_{\tau} e_{z} \times \nabla_{\tau}\left(\log \left(n_{\mathrm{wg}}^{2}\right)\right) \cdot \hat{\mathbf{z}},\right.\end{array}\right.$

where $\nabla_{\tau}$ (resp. $\Delta_{\tau}$ ) stands for the two-dimensional gradient $\nabla_{\tau} u=\left(\frac{\partial u}{\partial x}, \frac{\partial u}{\partial y}\right)^{T}$ (resp. the two-dimensional Laplacian $\left.\Delta_{\tau} u=\frac{\partial^{2} u}{\partial x^{2}}+\frac{\partial^{2} u}{\partial y^{2}}\right)$, and we have introduced the $2 \mathrm{~d}$ curl operator $\left(u_{x}, u_{y}\right)^{T} \times\left(v_{x}, v_{y}\right)^{T}:=\left(u_{x} v_{y}-\right.$ $\left.u_{y} v_{x}\right) \hat{\mathbf{z}}$. The transverse components $\mathbf{e}_{\tau j}$ and $\mathbf{h}_{\tau j}$ are recovered from $e_{z j}$ and $h_{z j}$ via explicit relations; see [66, Equation (30-6)].

As regards the radiating modes - gathered in the fields $\mathbf{E}_{\mathrm{rad}}$ and $\mathbf{H}_{\mathrm{rad}}$ featured in (2.11) - they are related to the essential spectrum of the operator (2.13); since these modes do not retain energy in the vicinity of the device, they are quite undesirable for our purpose and we do not elaborate further on them.

In practice the eigenvalue problem (2.13) is solved thanks to a numerical solver. Examples of guided modes are shown in Fig. 3.

Let us finally point out a crucial property of modes, namely their orthogonality (see for example [66, Section 31-3]); under appropriate normalization, the following relations hold, as a consequence of the so-called 

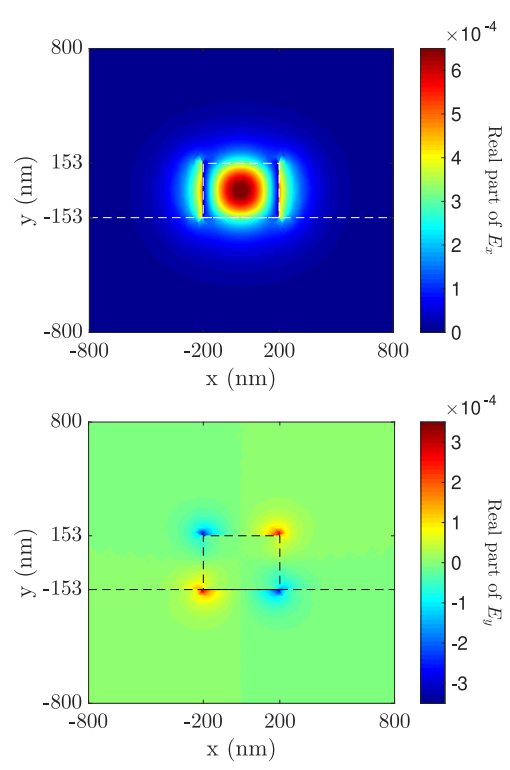

(a) First TE mode (TE $)$
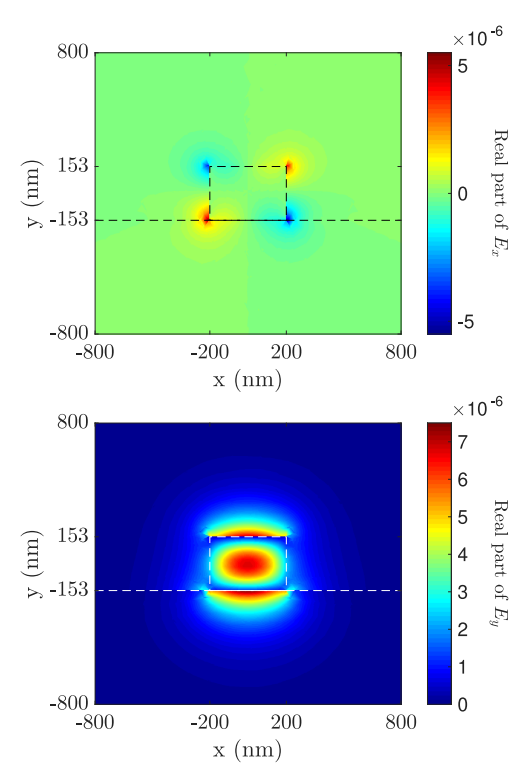

(b) First $T M$ mode $\left(T M_{0}\right)$
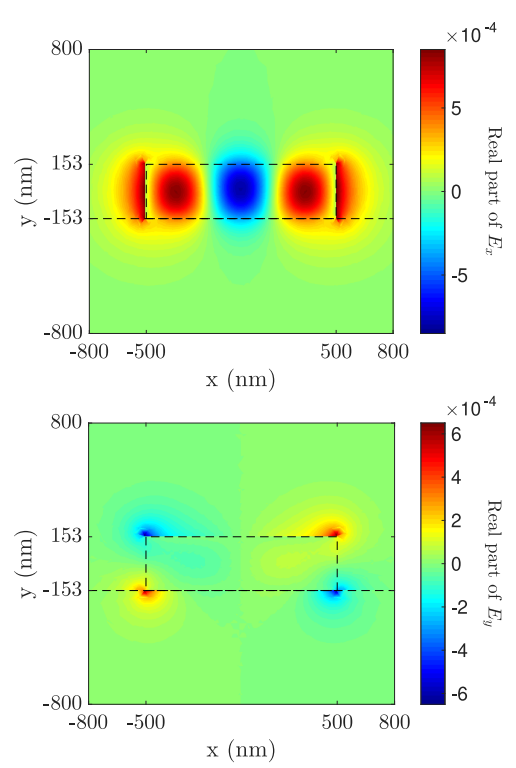

(c) Third TE mode $\left(T E_{2}\right)$

Figure 3 - Some guided mode inside two different waveguides with a silicon core of size $400 \times 306 \mathrm{~nm}$ and $1000 \times 306 \mathrm{~nm}$, mounted on a silica layer and surrounded by air at wavelength $\lambda=1.55 \mu \mathrm{m}$.

Reciprocity Theorem and (2.12):

$$
\forall j, l=-N, \ldots,-1,1, \ldots, N, \forall|j| \neq|l|, \quad \int_{\mathcal{S}}\left[\mathbf{E}_{j} \times \mathbf{H}_{l}^{*}\right] \cdot \hat{\mathbf{z}} d s=\int_{\mathcal{S}}\left[\mathbf{e}_{j} \times \mathbf{h}_{l}^{*}\right] \cdot \hat{\mathbf{z}} d s=\left\{\begin{array}{cl}
0 & \text { if }|j| \neq|l|, \\
2 & \text { if } l=|j|, \\
-2 & \text { if } l=-|j|,
\end{array}\right.
$$

where ${ }^{*}$ refers to complex conjugation. In particular, when this normalization holds, it is easily seen that the coefficients $a_{j}$ in (2.11) have the following expression:

$$
\forall j=1, \ldots, N, a_{j}=\frac{1}{4} \int_{\mathcal{S}}\left(\mathbf{E} \times \mathbf{H}_{j}^{*}+\mathbf{E}_{j}^{*} \times \mathbf{H}\right) \cdot \hat{\mathbf{z}} d s \text { and } a_{-j}=\frac{1}{4} \int_{\mathcal{S}}\left(\mathbf{E} \times \mathbf{H}_{j}^{*}-\mathbf{E}_{j}^{*} \times \mathbf{H}\right) \cdot \hat{\mathbf{z}} d s .
$$

\section{Remark 2.1.}

- We have been deliberately elusive as far as the mathematical setting behind the eigenvalue problem (2.13) is concerned. Let us simply mention that it has to be posed in an adapted functional space, and should be complemented with adequate boundary conditions to simulate the decay of the fields at infinity which resemble very much those used in Section 2.4.2 below.

- A waveguide supporting only one (resp. several) forward propagating guided mode (i.e. $N=1$ in (2.11)) is called mono-mode (resp. multi-mode).

- The guided modes of a waveguide usually carry a label of the form $T E_{j}$ or $T M_{j}$, where the polarization of the mode is transverse electric (TE) (resp. transverse magnetic (TM)) if the triplet $\left(E_{x}, H_{y}, E_{z}\right)$ contains more energy than $\left(H_{x}, E_{y}, H_{z}\right)$ (resp. if it is the other way around), and where $j=0, \ldots$ refers to the index of the corresponding eigenvalue. For instance, $T E_{0}$ (resp. TM $M_{0}$ ) refers to the first forwardpropagating guided mode (i.e. with smallest associated eigenvalue within the $\beta_{j}, j=1, \ldots, N$ ) which is transverse electric (resp. transverse magnetic).

\subsection{Boundary conditions}

In this section, we describe the mathematical formulation of the boundary conditions imposed on the electric field $\mathbf{E}$, corresponding to the injection of one particular mode at the entrance boundary $\Gamma_{\text {in }}$ and to its decay at infinity. Although commonly used, these boundary conditions are not so easily found as is in the literature and we briefly discuss them here for the sake of completeness. 


\subsubsection{Injection of a particular mode at the entrance port $\Gamma_{\mathrm{in}}$}

In the setting of Section 2.1 (see also Fig. 1), light is injected into the nanophotonic component at the entrance port $\Gamma_{\text {in }} \subset \partial \mathcal{D}$ under the form of the $m^{\text {th }}$ mode $\mathbf{E}_{m}$ of the corresponding input waveguide (which is assumed to be infinite, with axis $\hat{\mathbf{z}}$ and transverse section $\mathcal{S}$ ). In the literature, a boundary condition accounting for this effect is usually expressed as an impedance condition:

$$
\mathbf{n} \times \nabla \times \mathbf{E}+\gamma(\mathbf{E})=\mathbf{n} \times \nabla \times \mathbf{E}_{m}+\gamma\left(\mathbf{E}_{m}\right)=\mathbf{U}_{\text {inc }} \text { on } \Gamma_{\mathrm{in}},
$$

where $\mathbf{n}$ is the unit normal vector to $\partial \mathcal{D}$, pointing outward $\mathcal{D}\left(\mathbf{n}=-\hat{\mathbf{z}}\right.$ on $\Gamma_{\text {in }}$ in our context), $\mathbf{U}_{\text {inc }}$ is an appropriate source term and $\gamma$ is a linear operator; the perhaps simplest choice about $\gamma$ is:

$$
\gamma(\mathbf{E})=i \alpha \mathbf{n} \times \mathbf{E} \times \mathbf{n},
$$

for some real constant $\alpha>0$, a common practice when it comes to imposing absorbing boundary conditions. Depending on the context, other possibilities include the linear, inhomogeneous and isotropic relation

$$
\gamma(\mathbf{E})=i \omega k n \mathbf{n} \times \mathbf{E} \times \mathbf{n},
$$

as suggested in [39] or the inhomogeneous and anisotropic relation

$$
\gamma(\mathbf{E})=i \omega \mu_{0} \mathbf{n} \times\left(Z^{-1} \mathbf{E} \times \mathbf{n}\right),
$$

involving a real-valued, symmetric positive definite matrix $Z$, as in [69].

In our applications, the device in $\mathcal{D}_{\text {opt }}$ will inevitably entail reflected waves in the entrance port, and using the previous type of boundary conditions (which implicitly assume that there are none) would only account for erroneous incoming fields. For this reason, we rely on a non local boundary condition, which we briefly describe below, referring to [73, Section 6.3] or [40, Section 11.1.2] for full details. The main idea is to express the reflected electric field from the device into the waveguide in terms of the (unknown) total field. Recalling the orthogonal decomposition (2.11) of the electric and magnetic fields $\mathbf{E}$ and $\mathbf{H}$ inside the waveguide, we require that the only forward propagating mode present in there be $\mathbf{E}_{m}$, and that the radiated field $\mathbf{E}_{\mathrm{rad}}$ vanishes, while imposing nothing particular for the backward-propagating modes, i.e.

$$
\mathbf{E}(\mathbf{x})=\mathbf{E}_{m}(\mathbf{x})+\sum_{j=1}^{N} a_{-j} \mathbf{E}_{-j}(\mathbf{x}) .
$$

Using the orthogonality relations $(2.14)$, the coefficients $a_{-j} \in \mathbb{C}$ read:

$$
a_{-j}=-\frac{1}{2} \int_{\mathcal{S}}\left[\left(\mathbf{E}-\mathbf{E}_{\mathrm{m}}\right) \times \mathbf{H}_{-j}^{*}\right] \cdot \hat{\mathbf{z}} d s .
$$

Looking back to (2.17), we now obtain:

$$
\hat{\mathbf{z}} \times \nabla \times \mathbf{E}=\hat{\mathbf{z}} \times \nabla \times \mathbf{E}_{m}-\frac{1}{2} \sum_{j=1}^{N} \hat{\mathbf{z}} \times \nabla \times \mathbf{E}_{-j} \int_{\mathcal{S}}\left[\left(\mathbf{E}-\mathbf{E}_{m}\right) \times \mathbf{H}_{-j}^{*}\right] \cdot \hat{\mathbf{z}} d s,
$$

whence, using (2.2) and the orthogonality relations (2.14), it follows:

$$
\begin{aligned}
\hat{\mathbf{z}} \times \nabla \times \mathbf{E}+\frac{i \omega \mu}{2} \sum_{0} \sum_{j=1}^{N} \hat{\mathbf{z}} \times \mathbf{H}_{-j} \int_{\mathcal{S}}\left[\mathbf{E} \times \mathbf{H}_{-j}^{*}\right] \cdot \hat{\mathbf{z}} d s & = \\
& i \omega \mu_{0} \hat{\mathbf{z}} \times \mathbf{H}_{m}+\frac{i \omega \mu_{0}}{2} \hat{\mathbf{z}} \times \mathbf{H}_{-m} \int_{\mathcal{S}}\left[\mathbf{E}_{m} \times \mathbf{H}_{-m}^{*}\right] \cdot \hat{\mathbf{z}} d s .
\end{aligned}
$$

Finally, remarking from (2.12) that

$$
\hat{\mathbf{z}} \times \mathbf{H}_{-j}=-\hat{\mathbf{z}} \times \mathbf{H}_{j} \text {, and }\left[\mathbf{E} \times \mathbf{H}_{-j}^{*}\right] \cdot \hat{\mathbf{z}}=-\left[\mathbf{E} \times \mathbf{H}_{j}^{*}\right] \cdot \hat{\mathbf{z}} \text {, for } j=1, \ldots, N,
$$

we obtain

$$
\hat{\mathbf{z}} \times \nabla \times \mathbf{E}+\frac{i \omega \mu_{0}}{2} \sum_{j=1}^{N} \hat{\mathbf{z}} \times \mathbf{H}_{j} \int_{\mathcal{S}}\left[\mathbf{E} \times \mathbf{H}_{j}^{*}\right] \cdot \hat{\mathbf{z}} d s=2 i \omega \mu_{0} \hat{\mathbf{z}} \times \mathbf{H}_{m},
$$


a relation which is of the form $(2.16)$ with the definitions:

$$
\begin{gathered}
\gamma(\mathbf{E})=\frac{1}{2} \sum_{j=1}^{N} i \omega \mu_{0} \hat{\mathbf{z}} \times \mathbf{H}_{j} \int_{\mathcal{S}}\left[\mathbf{E} \times \mathbf{H}_{j}^{*}\right] \cdot \hat{\mathbf{z}} d s, \\
\mathbf{U}_{\mathrm{inc}}=2 i \omega \mu_{0} \hat{\mathbf{z}} \times \mathbf{H}_{m} .
\end{gathered}
$$

In practice, most of our waveguides will turn out to be mono-mode; then in the sum in (2.18), the sole fundamental mode $j=1$ appears.

\subsubsection{Approximation of the decay conditions at infinity using a Perfectly Matched Layer}

When the Maxwell equation (2.6) for the electric field is considered in the whole ambient space (that is, in a homogeneous domain which is infinite in all directions), it is customary to account for the behavior of $\mathbf{E}$ at infinity by using the so-called Silver-Müller radiation condition:

$$
\lim _{|\mathbf{x}| \rightarrow \infty}|\mathbf{x}|\left(\nabla \times \mathbf{E} \times \frac{\mathbf{x}}{|\mathbf{x}|}-i \omega \mathbf{E}\right)=0
$$

Let us mention that in the situation depicted in Fig. 1, the presence of waveguides at the border of the domain imply that (2.20) is not exactly the correct boundary condition (see [56] for a more in-depth discussion and analysis on this subject).

In any event, since our numerical calculations take place in the bounded computational domain $\mathcal{D}$, there is the need to impose artificial boundary conditions on $\partial \mathcal{D}$ which mimic (2.20) without inducing too much reflection.

In the following, we rely on the Perfectly Matched Layer (PML) method to tackle this issue, as proposed initially in [10]. This method does not involve a new type of boundary condition on $\partial \mathcal{D}$ so to speak; it rather relies on a thin, "perfectly matched" layer $\mathcal{D}_{\mathrm{PML}} \subset \mathcal{D}$ made of an artificial, absorbing material with the following properties:

1. Any wave, regardless of its angle of incidence, can penetrate inside $\mathcal{D}_{\mathrm{PML}}$ without causing reflection inside $\mathcal{D}$.

2. The amplitude of any wave propagating inside $\mathcal{D}_{\mathrm{PML}}$ decreases exponentially fast to 0 .

If both properties are fulfilled, imposing any type of homogeneous boundary conditions on $\partial \mathcal{D}$ for the electric field $\mathbf{E}$ - for instance the usual Dirichlet condition $\mathbf{n} \times \mathbf{E}=0$ - ensures a suitable approximation of the radiation condition $(2.20)$.

Let us now briefly discuss the construction of such a perfectly matched layer, referring to [40, Section 9.6] or [53, Section 13.5.3.1] for details. Recalling that the computational domain $\mathcal{D}$ is a box with size $2 w_{x} \times 2 w_{y} \times 2 w_{z}$ (see Fig. 1 ), the perfectly matched layer $\mathcal{D}_{\mathrm{PML}}$ is defined by:

$$
\mathcal{D}_{\mathrm{PML}}=\mathcal{D} \backslash\left[-w_{x}+w_{\mathrm{PML}}, w_{x}-w_{\mathrm{PML}}\right] \times\left[-w_{y}+w_{\mathrm{PML}}, w_{y}-w_{\mathrm{PML}}\right] \times\left[-w_{z}+w_{\mathrm{PML}}, w_{z}-w_{\mathrm{PML}}\right],
$$

where $w_{\mathrm{PML}}$ is the "small" thickness of the layer. We then define for each component $\iota=x, y, z$ :

$$
\sigma_{\iota}(s)=\left\{\begin{array}{rlll}
1 & \text { if } & |s| \leq w_{\iota}-w_{\mathrm{PML}} \\
1+\frac{i \lambda}{k} \frac{1}{w_{\iota}-|s|} & \text { if } & |s|>w_{\iota}-w_{\mathrm{PML}}
\end{array}\right.
$$

and thence the anisotropic tensor $\Lambda$ by:

$$
\Lambda(\mathbf{x})=\left(\begin{array}{ccc}
\sigma_{x}^{-1}(x) \sigma_{y}(y) \sigma_{z}(z) & 0 & 0 \\
0 & \sigma_{x}(x) \sigma_{y}(y)^{-1} \sigma_{z}(z) & 0 \\
0 & 0 & \sigma_{x}(x) \sigma_{y}(y) \sigma_{z}(z)^{-1}
\end{array}\right), \mathbf{x} \in \mathcal{D} .
$$

In particular, $\Lambda(\mathbf{x})$ coincides with the identity when $\mathbf{x} \notin \mathcal{D}_{\mathrm{PML}}$.

Finally, the electric field $\mathbf{E}$ is sought as the solution to (2.6), in which $\varepsilon$ and $\mu$ are replaced by the tensors fields $\varepsilon \Lambda$ and $\mu \Lambda$, respectively; see (2.23) below. 


\subsection{The complete variational framework}

The Maxwell equations, complemented with the mode injection boundary condition of Section 2.4.1, rearranged to take into account the "perfectly matched" layer of Section 2.4.2 lead to the following system:

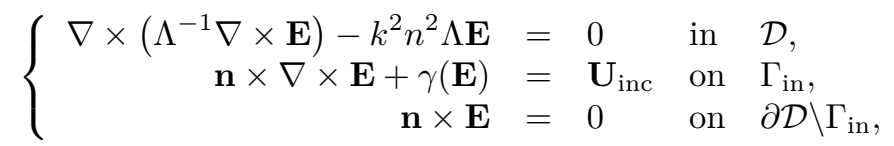

where $\mathbf{U}_{\text {inc }}$ is associated via (2.16) to the injection of the $m^{\text {th }}$ mode $\left(\beta_{m}, \mathbf{E}_{m}\right)$ of the incoming waveguide, and the matrix $\Lambda$ is defined in (2.22).

The numerical resolution of this system relies on an associated variational formulation; the latter brings into play the functional space

$$
\mathcal{V}=\{\mathbf{E} \in H(\operatorname{curl}, \mathcal{D}), \mathbf{n} \times \mathbf{E}=0 \text { on } \partial \mathcal{D}\}
$$

It reads search for the unique $\mathbf{E}_{\Omega} \in \mathcal{V}$ such that:

$$
\forall \boldsymbol{\phi} \in \mathcal{V}, \quad \int_{\mathcal{D}}\left(\Lambda^{-1} \nabla \times \mathbf{E} \cdot \nabla \times \boldsymbol{\phi}^{*}-k^{2} n^{2} \Lambda \mathbf{E} \cdot \boldsymbol{\phi}^{*}\right) d \mathbf{x}+\int_{\Gamma_{\mathrm{in}}}\left(\mathbf{U}_{\mathrm{inc}}-\gamma(\mathbf{E})\right) \cdot\left(\mathbf{n} \times \boldsymbol{\phi}^{*} \times \mathbf{n}\right) d s=0
$$

where the integral on the edge $\Gamma_{\text {in }}$ in the above equation makes sense owing to [53, Theorem 3.31]. We refer to [53] about the well-posedness of (2.25) and about its numerical discretization.

\section{Shape optimization of nanophotonic devices}

In this section, we introduce the shape and topology optimization problem considered in this article as well as the ingredients from shape sensitivity analysis required in its treatment.

\subsection{Presentation of the shape optimization problem}

Our purpose is to optimize the shape of the phase $\Omega$ filled with core material within the design domain $\mathcal{D}_{\text {opt }}$; see Fig. 1. The shape and topology optimization problem under scrutiny is of the form:

$$
\max _{\Omega \in \mathcal{U}_{\mathrm{ad}}} J(\Omega)
$$

where $J(\Omega)$ is a performance criterion depending on the domain $\Omega$ and $\mathcal{U}_{\text {ad }}$ is a set of admissible shapes; namely (see (2.1)):

$$
\mathcal{U}_{\text {ad }}=\left\{\Omega \subset \mathcal{D}_{\text {opt }}, \Omega \text { is invariant in the } y \text { direction }\right\} .
$$

Notice that, contrary to the prevailing convention in the shape and topology optimization literature, our goal is to maximize the considered objective function $J(\Omega)$.

We shall consider various objective functions, all related to either the output power of the device, or to the structure of the electromagnetic field resulting from the action of the device, that is, the fraction of energy carried by one particular mode; see Section 6 for the precise objectives used in this work. Both quantities are defined in terms of the Poynting vector

$$
\Pi_{\Omega}:=\frac{1}{2} \mathcal{R} e\left(\mathbf{E}_{\Omega} \times \mathbf{H}_{\Omega}^{*}\right)
$$

where $\mathbf{E}_{\Omega}$ and $\mathbf{H}_{\Omega}$ are the solutions to (2.2) and (2.4) when the core material occupies the region $\Omega \subset \mathcal{D}_{\text {opt }}$ The electromagnetic power crossing an arbitrary oriented surface $\Gamma_{\text {obj }} \subset \mathcal{D}$ with unit normal vector $\mathbf{n}$ then reads:

$$
J_{\mathrm{p}}(\Omega)=\int_{\Gamma_{\mathrm{obj}}} \Pi_{\Omega} \cdot \mathbf{n} d s
$$

In the applications of this article, the surface $\Gamma_{\text {obj }}$ stands for the port of an output waveguide connected to $\mathcal{D}_{\text {opt }}$, whose longitudinal direction is oriented by $\hat{\mathbf{z}}=\mathbf{n}$. This power may then be decomposed onto the modes 
of this waveguide, according to (2.11). Introducing the fraction $J_{\mathrm{o}, m}(\Omega)$ of this energy which is conveyed by the $m$-th mode $\left(\beta_{-m}, \mathbf{E}_{-m}\right)$, the following relation holds:

$$
J_{p}(\Omega)=\sum_{m=1}^{N} J_{\mathrm{o}, m}(\Omega)-\sum_{m=1}^{N} J_{\mathrm{o},-m}(\Omega)
$$

Using the decomposition (2.11) and the orthogonality relation (2.14), $J_{\mathrm{o}, m}(\Omega)$ may be written as the following overlap integral:

$$
\begin{aligned}
J_{\mathrm{o}, m}(\Omega) & =\left|\frac{1}{4} \int_{\Gamma_{\mathrm{obj}}}\left(\mathbf{E}_{\Omega} \times \mathbf{H}_{m}^{*}+\mathbf{E}_{m}^{*} \times \mathbf{H}_{\Omega}\right) \cdot \mathbf{n} d s\right|^{2} \\
& =\left|\frac{1}{4} \int_{\Gamma_{\mathrm{obj}}}\left(\mathbf{E}_{\Omega} \times \mathbf{H}_{m}^{*}-\frac{i}{\omega \mu_{0}} \mathbf{E}_{m}^{*} \times\left(\nabla \times \mathbf{E}_{\Omega}\right)\right) \cdot \mathbf{n} d s\right|^{2}
\end{aligned}
$$

where we have used (2.2) to pass from the first line to the second one.

Remark 3.1. In principle, the problem (3.1) could be complemented with constraints, e.g. on the volume of the core phase $\Omega$, without much change to the forthcoming developments.

\subsection{Differentiation with respect to the domain using Hadamard's boundary vari- ation method}

The resolution of our shape optimization problems (3.1) relies on a gradient-based algorithm; this raises the need to calculate the derivatives of the featured objective functions with respect to the domain, a notion which may be given several different meanings.

In our context, we rely on Hadamard's boundary variation method; see [34] for the original work, and $[1,35,54]$ for more recent developments and expositions. In a nutshell, variations of a given reference shape $\Omega$ are considered under the form:

$$
\Omega_{\boldsymbol{\theta}}:=(\operatorname{Id}+\boldsymbol{\theta})(\Omega), \boldsymbol{\theta} \in W^{1, \infty}\left(\mathbb{R}^{3}, \mathbb{R}^{3}\right),\|\boldsymbol{\theta}\|_{W^{1, \infty}\left(\mathbb{R}^{3}, \mathbb{R}^{3}\right)}<1 .
$$

Accordingly, a function $J(\Omega)$ of the domain is shape differentiable at a particular shape $\Omega$ if the underlying mapping $\boldsymbol{\theta} \mapsto J\left(\Omega_{\boldsymbol{\theta}}\right)$, from $W^{1, \infty}\left(\mathbb{R}^{3}, \mathbb{R}^{3}\right)$ into $\mathbb{R}$, is Fréchet differentiable at $\boldsymbol{\theta}=0$; the corresponding Fréchet derivative $\boldsymbol{\theta} \mapsto J^{\prime}(\Omega)(\boldsymbol{\theta})$ is the shape derivative of $J(\Omega)$ at $\Omega$. In other words, the following expansion holds:

$$
J\left(\Omega_{\boldsymbol{\theta}}\right)=J(\Omega)+J^{\prime}(\Omega)(\boldsymbol{\theta})+o(\boldsymbol{\theta}), \text { where } \lim _{\boldsymbol{\theta} \rightarrow 0} \frac{o(\boldsymbol{\theta})}{\|\boldsymbol{\theta}\|_{W^{1, \infty}\left(\mathbb{R}^{3}, \mathbb{R}^{3}\right)}}=0 .
$$

In the case of a "smooth enough" objective $J(\Omega)$, the shape derivative $J^{\prime}(\Omega)(\boldsymbol{\theta})$ has the particular structure:

$$
J^{\prime}(\Omega)(\boldsymbol{\theta})=\int_{\partial \Omega} g_{\Omega} \boldsymbol{\theta} \cdot \mathbf{n}_{\Omega} d s
$$

where $g_{\Omega}: \partial \Omega \rightarrow \mathbb{R}$ is a scalar field and $\mathbf{n}_{\Omega}: \partial \Omega \rightarrow \mathbb{S}^{2}$ is the unit normal vector to $\partial \Omega$, pointing outward $\Omega$; see [35] for a precise statement of this Structure Theorem. From (3.8), an ascent direction for $J(\Omega)$ is revealed as the gradient associated to the derivative $\boldsymbol{\theta} \mapsto J^{\prime}(\Omega)(\boldsymbol{\theta})$ in $(3.8)$ via the $L^{2}(\partial \Omega)$ inner product, that is:

$$
\boldsymbol{\theta}=g_{\Omega} \mathbf{n}_{\Omega} .
$$

Unfortunately, this choice may be ill-suited. One reason for this fact is that (3.9) only determines the values of $\boldsymbol{\theta}$ on $\partial \Omega$, while our numerical algorithm for describing evolution of shapes requires that the considered ascent direction be defined on the whole design domain $\mathcal{D}_{\text {opt }}$ (see Section 5 ). Moreover, the scalar field $g_{\Omega}$ may be quite irregular, thus jeopardizing the accuracy of the numerical process. To circumvent both drawbacks at the same time, a convenient remedy consists in considering in (3.9) the gradient associated to $J^{\prime}(\Omega)$ via a different inner product than that of $L^{2}(\partial \Omega)$; see $[12,21]$. More precisely, we solve the variational problem:

$$
\text { Find } \tilde{v} \in V \text { s.t. } \forall w \in V, a(\tilde{v}, w)=\int_{\partial \Omega} g_{\Omega} w d s
$$


relying on the following choice of Hilbert space $V$ and inner product $a(\cdot, \cdot)$ :

$$
V=H^{1}\left(\mathcal{D}_{\mathrm{opt}}\right), a(v, w)=\int_{\mathcal{D}_{\mathrm{opt}}}(\alpha \nabla v \cdot \nabla w+v w) d x
$$

where $\alpha>0$ is a small parameter; in practice, $\alpha$ is of the order of $\Delta x^{2}$, where $\Delta x$ stands for the mesh size. From (3.7) and (3.10), an ascent direction for $J(\Omega)$ is revealed as $\boldsymbol{\theta}=\tilde{v} \mathbf{n}_{\Omega}$, in which the normal vector $\mathbf{n}_{\Omega}$ has been extended to $\mathcal{D}_{\text {opt }}$ as a whole.

\section{Remark 3.2.}

- As any gradient-based optimization algorithm, the above maximization scheme will inevitably result in a local optimum of the program (3.1).

- In practice, deformations $\boldsymbol{\theta}$ are restricted to a subset $\Theta_{\mathrm{ad}} \subset W^{1, \infty}\left(\mathbb{R}^{3}, \mathbb{R}^{3}\right)$ of admissible deformations, in such a way that variations $\Omega_{\boldsymbol{\theta}}$ of admissible shapes stay admissible. In our context, the vector fields $\boldsymbol{\theta} \in \Theta_{\mathrm{ad}}$ are smooth and invariant in the $y$ direction: $\partial_{y} \boldsymbol{\theta}=0$; see (3.2).

\subsection{Shape derivatives of the considered objective functional}

\subsubsection{The 'exact' shape derivative of $J(\Omega)$}

The following theorem provides the shape derivative of the objective function (3.5); see Appendix A for a sketch of proof.

Theorem 3.1. The functional $J_{\mathrm{o}, m}(\Omega)$ defined by (3.5) is shape differentiable at any admissible shape $\Omega \in \mathcal{U}_{\mathrm{ad}}$ and its shape derivative reads:

$$
J_{\mathrm{o}, m}^{\prime}(\Omega)(\boldsymbol{\theta})=\int_{\partial \Omega} g_{\Omega} \boldsymbol{\theta} \cdot \mathbf{n}_{\Omega} d s,
$$

where

$$
\begin{aligned}
g_{\Omega}=k^{2} \mathcal{R} e\left[\left(n_{\text {clad }}^{2}-n_{\text {core }}^{2}\right)\left(\mathbf{n}_{\Omega} \times \mathbf{E}_{\Omega} \times \mathbf{n}_{\Omega}\right) \cdot\left(\mathbf{n}_{\Omega} \times \mathbf{A}_{\Omega}^{*} \times \mathbf{n}_{\Omega}\right)\right. \\
\left.-\left(n_{\text {clad }}^{-2}-n_{\text {core }}^{-2}\right)\left(\left(n_{\Omega}^{2} \mathbf{E}_{\Omega}\right) \cdot \mathbf{n}_{\Omega}\right)\left(\left(n_{\Omega}^{2} \mathbf{A}_{\Omega}^{*}\right) \cdot \mathbf{n}_{\Omega}\right)\right],
\end{aligned}
$$

and the adjoint state $\mathbf{A}_{\Omega}$ is the unique solution in $\mathcal{V}$ to the following variational problem:

$$
\begin{aligned}
\int_{\mathcal{D}} \Lambda^{-1} & \nabla \times \mathbf{A} \cdot \nabla \times \phi^{*}-k^{2} n_{\Omega}^{2} \Lambda \mathbf{A} \cdot \boldsymbol{\phi}^{*} d \mathbf{x}-\int_{\Gamma} \gamma(\mathbf{A}) \cdot \mathbf{n} \times \boldsymbol{\phi}^{*} \times \mathbf{n} d s \\
& =-\frac{1}{8}\left(\int_{\Gamma_{\text {out }}}\left(\mathbf{E}_{\Omega} \times \mathbf{H}_{m}^{*}+\mathbf{E}_{m}^{*} \times \mathbf{H}_{\Omega}\right) \cdot \mathbf{n} d s\right)^{*} \int_{\Gamma_{\text {out }}}\left(\phi \times \mathbf{H}_{m}^{*}-\frac{i}{\omega \mu_{0}} \mathbf{E}_{m}^{*} \times \nabla \times \boldsymbol{\phi}\right) \cdot \mathbf{n} d s .
\end{aligned}
$$

\section{Remark 3.3.}

- The expression (3.13) is well-defined since the transmission conditions at the interface $\partial \Omega$ between the two phases with different refractive indices $n_{\text {core }}$ and $n_{\text {clad }}$ (which are implicitly comprised in the variational formulation (2.25)) imply the continuity of the tangential component $\mathbf{n} \times \mathbf{E}_{\Omega}$ of $\mathbf{E}_{\Omega}$ and of the normal component $n_{\Omega}^{2} \mathbf{E}_{\Omega} \cdot \mathbf{n}$ of the electric displacement $n_{\Omega}^{2} \mathbf{E}_{\Omega}$; see [53] and Appendix A about this point.

- Taking into account the $y$-invariance of admissible shapes $\Omega \in \mathcal{U}_{\mathrm{ad}}$ (see (2.1)), and the independence of admissible vector field $\boldsymbol{\theta} \in \Theta_{\mathrm{ad}}$ from the variable y (see Remark 3.2), (3.12) rewrites:

$$
J_{\mathrm{o}, m}^{\prime}(\Omega)(\boldsymbol{\theta})=\int_{\partial \widehat{\Omega}} \widehat{g}_{\Omega}(x, z) \boldsymbol{\theta}(x, z) \cdot \mathbf{n}_{\Omega}(x, z) d x d z \text {, where } \widehat{g}_{\Omega}(x, z):=\int_{0}^{h} g_{\Omega}(x, y, z) d y .
$$




\subsubsection{Numerical approximation of the shape derivative}

The shape derivative (3.13) of the functional $J_{\mathrm{o}, m}(\Omega)$ is unfortunately difficult to handle in numerical practice. Indeed, it involves the values of the normal components of $n_{\Omega}^{2} \mathbf{E}_{\Omega}$ and $n_{\Omega}^{2} \mathbf{A}_{\Omega}$ on the interface $\partial \Omega$ - these quantities being continuous across $\partial \Omega$; see Remark 3.3. In our numerical framework, however (see Section 5.1 below), the shape $\Omega$ is not explicitly discretized at each stage of the optimization process: the interface $\partial \Omega$ does not coincide with faces of the tetrahedral mesh of $\mathcal{D}$. It follows that the discretized version of $\mathbf{E}_{\Omega}$ is continuous inside each tetrahedron, so that the discrete counterpart to $n_{\Omega}^{2} \mathbf{E}_{\Omega}$ (or $n_{\Omega}^{2} \mathbf{A}_{\Omega}$ ) is naturally discontinuous on $\partial \Omega$; this entails large errors in the numerical evaluation of (3.13).

To alleviate this problem, we rely on the method from our previous work [4] to construct a consistent approximation $J_{\mathrm{o}, m, \eta}(\Omega)$ of $J_{\mathrm{o}, m}(\Omega)$, controlled by a small parameter $\eta>0$, whose shape derivative is easier to calculate in numerical practice. This method relies on a smoothed version $n_{\Omega, \eta}$ of the exact refractive index $n_{\Omega}$, which is constructed as follows:

$$
n_{\Omega, \eta}^{2}(\mathbf{x})=n_{\text {core }}^{2}+\left(n_{\text {clad }}^{2}-n_{\text {core }}^{2}\right) h_{\eta}\left(d_{\Omega}(\mathbf{x})\right) .
$$

In the above formula, $d_{\Omega}(\mathbf{x})$ is the signed distance function to $\Omega$, defined by:

$$
\forall \mathbf{x} \in \mathbb{R}^{3}, d_{\Omega}(\mathbf{x})=\left\{\begin{array}{cl}
-d(\mathbf{x}, \partial \Omega) & \text { if } \mathbf{x} \in \Omega, \\
0 & \text { if } \mathbf{x} \in \partial \Omega, \\
d(\mathbf{x}, \partial \Omega) & \text { if } \mathbf{x} \in \mathbb{R}^{3} \backslash \bar{\Omega}
\end{array}\right.
$$

where $d(\mathbf{x}, \partial \Omega)=\min _{\mathbf{x}^{\prime} \in \partial \Omega}\left|\mathbf{x}-\mathbf{x}^{\prime}\right|$ is the usual Euclidean distance between $\mathbf{x}$ and $\partial \Omega$. The function $h_{\eta}$ in (3.16) is a $\mathcal{C}^{2}$ approximation of the Heaviside function:

$$
\forall t \in \mathbb{R}, h_{\eta}(t)=\left\{\begin{array}{cl}
0 & \text { if } t<-\eta, \\
\frac{1}{2}\left(1+\frac{t}{\eta}+\frac{1}{\pi} \sin \left(\frac{\pi t}{\eta}\right)\right) & \text { if }-\eta \leq t \leq \eta, \\
1 & \text { if } t>\eta
\end{array}\right.
$$

in which $\eta$ controls the degree of smoothing.

Since $n_{\Omega, \eta}$ is smooth on the whole design domain $\mathcal{D}_{\text {opt }}$, then so are the solutions $\mathbf{E}_{\Omega, \eta}, \mathbf{A}_{\Omega, \eta}$ to the approximate Maxwell systems obtained from (2.23) and (3.14) respectively, by replacing $n_{\Omega}$ with $n_{\Omega, \eta}$; see [20, Chapter IX]. Extending the conclusions of [4] to the present situation, one may prove that:

1. $\mathbf{E}_{\Omega, \eta}$ converges to $\mathbf{E}_{\Omega}$ and $\mathbf{A}_{\Omega, \eta}$ converges to $\mathbf{A}_{\Omega}$ in $\mathcal{V}$ as $\eta \rightarrow 0$

2. For given shape $\Omega \in \mathcal{U}_{\mathrm{ad}}$ and deformation $\boldsymbol{\theta} \in \Theta_{\mathrm{ad}}$, the shape derivative $J_{\mathrm{o}, m, \eta}^{\prime}(\Omega)(\boldsymbol{\theta})$ converges to $J_{\mathrm{o}, m, \eta}(\Omega)(\boldsymbol{\theta})$ as $\eta \rightarrow 0$. Since, for $\eta>0$ small enough,

$$
J_{\mathrm{o}, m, \eta}^{\prime}(\Omega)(\boldsymbol{\theta}) \approx \int_{\partial \Omega} k^{2}\left(n_{\text {clad }}^{2}-n_{\text {core }}^{2}\right) \mathcal{R} e\left(\mathbf{E}_{\Omega, \eta} \cdot \mathbf{A}_{\Omega, \eta}^{*}\right)\left(\boldsymbol{\theta} \cdot \mathbf{n}_{\Omega}\right) d s
$$

the latter quantity may be used as a consistent approximation of $J_{\mathrm{o}, m}^{\prime}(\Omega)$, and it is much easier to calculate in numerical practice.

Notice that such a regularization procedure is quite popular in the literature for accurate electromagnetism simulations but, to the best of our knowledge, without mathematically rigorous justification. See for instance [41] or [26], in which an anisotropic counterpart to (3.16) and (3.18) is used.

\section{Robustness in the optimization of nanophotonic devices}

The physical and geometrical properties of the considered nanophotonic components and its environment are characterized by data (the incoming wavelength, the refractive indices of the media at play, or the morphology of shapes itself, to name a few) which are in practice known with some uncertainty. The electromagnetic fields around nanophotonic devices, and thereby their physical performances, being very sensitive to these data, it is of utmost importance to optimize their shapes in such a way that their performances be robust with respect to such uncertainties, i.e. so that they retain an acceptable efficiency in a variety of fabrication or operating conditions. 
Optimization of shapes in a way which is robust to uncertainties has been a burning issue in shape and topology optimization lately. In the recent review [52], manufacturing uncertainties have been identified as the main stumbling block preventing the use of nanophotonic components at the industrial level, and studies towards alleviating these problems have been initiated [70, 24]. Beyond the field of nanophotonics, robustness issues in shape and topology optimization have been addressed from two fairly different viewpoints:

- When no information is available about the uncertain data but for a bound on their maximum amplitude, the worst value of the performance criterion under all possible uncertain data is optimized. These problems are generally way too difficult to be dealt with in utter generality, since their treatment inherently involves a bilevel optimization program; yet several particular situations or approximations have provided quite satisfactory answers $[2,8,15,33]$. The main drawback of such approaches is that they are generally too pessimistic: while the worst case scenario is likely never to happen in practice, the specific optimization of this situation may conduct to shapes with poor nominal performance.

- When more information is available about the statistics of the uncertain data (e.g. about its first- and second-order moments), probabilistic approaches may be considered for the minimization of the average value or the standard deviation of the performance criterion; see [48] for an overview. These approaches generally rely on very costly sampling strategies, such as Monte-Carlo, or collocation methods, involving a large number of evaluations of the considered cost function and its derivative; see for instance [47] and the references therein. Linearized approximations of such problems have been proposed [44, 3].

In this article, we rely on a simple sampling strategy for the robust worst-case optimization when small uncertainties are expected; this method is particularly well-suited in situations where the uncertain data lie in a low-dimensional space. Our approach is guided by the large CPU cost of the numerical resolution of systems of the form (2.23), which makes methods involving a large number of evaluations of the objective function and its derivative totally impractical in our context. The general principle of the method is presented in Section 4.1 in an abstract and formal way. Its particular application to deal with robustness with respect to the incoming wavelength and to the geometry of shapes themselves are discussed in Sections 4.2 and 4.3, respectively.

\subsection{A general abstract setting}

Our uncertain data are modelled by a parameter $\delta$ lying in a set $X$. In practice we assume that

$X$ is a ball with small radius $m>0$ in a low-dimensional vector space.

Denoting by $J_{\delta}(\Omega)$ the value of the considered objective functional when the physical data $\delta$ are observed, our purpose is to maximize the worst value of $J_{\delta}(\Omega)$ when $\delta$ runs through $X$ :

$$
\max _{\Omega} \min _{\delta \in X} J_{\delta}(\Omega) .
$$

Taking advantage of the hypothesis (4.1), the previous problem is approximated by

$$
\max _{\Omega} \min _{i=1, \ldots, N} J_{i}(\Omega), \text { where } J_{i}(\Omega):=J_{\delta_{i}}(\Omega),
$$

and the $\delta_{i}, i=1, \ldots, N$ constitute a suitable sampling of $X$. Hence, the problem (4.2) is reformulated as that (4.3) of maximizing the minimum value between a finite number of objective functions. In order to find an ascent direction for (4.3), we linearize each function $J_{i}(\Omega)$ in the neighborhood of the actual shape $\Omega$, so that (4.3) becomes:

$$
\max _{\boldsymbol{\theta}} \min _{i=1, \ldots, N} J_{i}(\Omega)+J_{i}^{\prime}(\Omega)(\boldsymbol{\theta})
$$

where $\boldsymbol{\theta}$ runs over the set of admissible perturbations (see (3.6) and Remark 3.2). Let us now introduce the shape gradients $\boldsymbol{\theta}_{i} \in V$ associated to the shape derivatives $J_{i}^{\prime}(\Omega)(\boldsymbol{\theta})$ via the identification problem (3.10). We then search for a solution $\boldsymbol{\theta}$ to (4.4) in restriction to the convex hull conv $\left\{\boldsymbol{\theta}_{i}\right\}$ where

$$
\operatorname{conv}\left\{\boldsymbol{\theta}_{i}\right\}:=\left\{\sum_{i=1}^{N} \alpha_{i} \boldsymbol{\theta}_{i}, 0 \leq \alpha_{i} \leq 1, \sum_{i=1}^{N} \alpha_{i}=1\right\} .
$$


In other terms, one solution $\boldsymbol{\theta}$ to our approximate worst-case optimization problem (4.4) is sought under the form $\boldsymbol{\theta}=\sum_{i=1}^{N} \alpha_{i} \boldsymbol{\theta}_{i}$, where $\boldsymbol{\alpha} \in[0,1]^{N}$ is the solution to the following linear program:

$$
\begin{array}{ll}
\max _{\boldsymbol{\alpha}, r} & r \\
\text { s.t. } & \boldsymbol{\alpha} \in[0,1]^{N}, r \in \mathbb{R}, \\
& \sum_{i=1}^{N} \alpha_{i}=1 \\
& J_{i}(\Omega)+\sum_{j=1}^{N} \alpha_{j} a\left(\boldsymbol{\theta}_{i}, \boldsymbol{\theta}_{j}\right)>r, i=1, \ldots, N,
\end{array}
$$

where $a(\cdot, \cdot)$ is the bilinear form defined in (3.11).

Remark 4.1. Let us mention a useful variant of (4.5) for finding an ascent direction $\boldsymbol{\theta}$ for (4.4): $\boldsymbol{\theta}$ is sought as a convex combination $\boldsymbol{\theta}=\sum_{j=1}^{M} \widetilde{\alpha}_{j} \boldsymbol{\theta}_{i_{j}}$ where the set $\mathcal{I}=\left\{i_{1}, \ldots, i_{M}\right\} \subset\{1, \ldots, N\}$ only retains the indices of the "smallest" values among the $J_{i}(\Omega),(i=1, \ldots, N)$, i.e.

$$
\mathcal{I}=\left\{i=1, \ldots, N, J_{i}(\Omega)<\min _{j=1, \ldots, N} J_{j}(\Omega)+\eta\right\}
$$

for a small tolerance parameter $\eta>0$. The corresponding optimization program then reads:

$$
\begin{array}{ll}
\underset{\widetilde{\boldsymbol{\alpha}}, r}{\max } & r \\
\text { s.t. } & \widetilde{\boldsymbol{\alpha}} \in[0,1]^{M}, r \in \mathbb{R}, \\
& \sum_{j=1}^{M} \widetilde{\alpha_{j}}=1, \\
& \sum_{j=1}^{M} \widetilde{\alpha_{j}} a\left(\boldsymbol{\theta}_{i_{j}}, \boldsymbol{\theta}_{i_{l}}\right)>r, \quad l=1, \ldots, M .
\end{array}
$$

Remark 4.2. The above strategy is solely based on a sampling $J_{i}(\Omega)=J_{\delta_{i}}(\Omega)$ of the perturbed functional $J_{\delta}(\Omega)$ at particular values $\delta=\delta_{i}, i=1, \ldots, N$, and on the derivative of the sampled functionals $\Omega \mapsto J_{i}(\Omega)$. In particular, it does not involve the sensitivity of the objective function with respect to the perturbations, that is, the derivative of the mapping $\delta \mapsto J_{\delta}(\Omega)$, which is a noticeable difference with the linearization method from our previous work [2, 3].

\subsection{Robustness with respect to the wavelength}

One of the perhaps most crucial aspects where robustness is desired in nanophotonics is related to the wavelength $\lambda$ (or equivalently the frequency $\omega=2 \pi c / \lambda$ ) of the light injected into the component at stake. Aiming at a performance which is little altered by small variations of the incoming wavelength is indeed a way to cope with the inaccuracy of the laser realizing the light injection, or simply to construct large bandwidth devices.

Using the notations of Section 4.1 the considered set $X$ of perturbations is the interval $[\bar{\lambda}-m, \bar{\lambda}+m] \subset \mathbb{R}$, where $\bar{\lambda}$ is the ideal operating wavelength, and $m>0$ is a user-defined tolerance for the range of wavelengths where the optimized design should retain good performances. Let us denote by $J_{\lambda}(\Omega)$ the value of the considered objective function at a particular shape $\Omega \in \mathcal{U}_{\text {ad }}$ when the operating wavelength equals $\lambda$; notice that $\lambda$ influences three parameters of the physical model $(2.23)$, namely the wavenumber $k=2 \pi / \lambda$, the optical index $n$ and the features $\gamma$ and $\mathbf{U}_{\text {inc }}$ used in the expression of the light injection boundary conditions; see (2.18) and (2.19). The worst-case shape optimization problem reads, when uncertainties of amplitude $m$ around the value $\bar{\lambda}$ are expected about the wavelength:

$$
\max _{\Omega \in \mathcal{U}_{\mathrm{ad}}} \min _{\lambda \in X} J_{\lambda}(\Omega)
$$

Following Section 4.1, the set $X$ is sampled as $\left\{\lambda_{i}\right\}_{i=1, \ldots, N}$, and (4.7) readily boils down to a program of the type (4.5), which is solved thanks to the methodology described in Section 4.1. 


\subsection{Robustness with respect to geometric uncertainties}

We now illustrate how the general framework of Section 4.1 may be adapted to impose the robustness of the optimized designs $\Omega$ with respect to uncertainties on their geometry. Uncertainties related to the etching fabrication process are considered in Section 4.3.1, and an extension of these ideas is used in Section 4.3.2 to deal with uncertainties caused by lithography.

\subsubsection{Robustness with respect to uncertainties caused by the etching process: an approach using dilation and erosion}

As we have mentioned in the introduction, under- or over-etching of the design $\Omega$ is likely to occur in the course of the etching fabrication process. In other terms, the fabricated shape is a uniform dilation or erosion $\Omega_{\delta}$ of $\Omega$,

$$
\Omega_{\delta}:=\left(\operatorname{Id}+\delta \mathbf{n}_{\Omega}\right)(\Omega),
$$

where $\delta$ is a real-valued parameter with small amplitude $|\delta|<m$; see $[65,70]$ for the use of this modelling in density-based topology optimization, Remark 4.3 below for an extension, and Fig. 4 for an illustration.

In this context, the robust optimization problem (4.3) of interest brings into play the perturbed functional $J_{\delta}(\Omega)$ whose expression reads:

$$
J_{\delta}(\Omega)=J\left(\Omega_{\delta}\right)=J\left(\left(\operatorname{Id}+\delta \mathbf{n}_{\Omega}\right)(\Omega)\right) .
$$

In particular, (4.3) involves the optimized shape $\Omega$ via a modified version $\Omega_{\delta}$ as for instance in the contribution [14]. In our way towards converting (4.3) into a linear program of the form (4.5), the shape derivative of the individual functions $J_{\delta}(\Omega)$ is needed; the latter is the purpose of the next proposition, whose proof is postponed to Appendix B.

Proposition 4.1. Let $\Omega \in \mathcal{U}_{\mathrm{ad}}$, and let $\delta>0$ be small enough so that $\left(\mathrm{Id}+\delta \mathbf{n}_{\Omega}\right)$ is a diffeomorphism. The functional $J_{\delta}(\Omega)$ is shape differentiable at $\Omega$ and its shape derivative reads:

$$
J_{\delta}^{\prime}(\Omega)(\boldsymbol{\theta})=\int_{\partial \Omega} g_{\Omega_{\delta}} \circ\left(\mathrm{Id}+\delta \mathbf{n}_{\Omega}\right) H\left(\boldsymbol{\theta}, \mathbf{n}_{\Omega}\right) d s,
$$

where

$$
H\left(\boldsymbol{\theta}, \mathbf{n}_{\Omega}\right):=\left|\operatorname{det}\left(\operatorname{Id}+\delta \nabla \mathbf{n}_{\Omega}\right)\right|\left(\left(\left(\operatorname{Id}+\delta \nabla \mathbf{n}_{\Omega}\right)^{-1} \mathbf{n}_{\Omega} \cdot \mathbf{n}_{\Omega}\right) \boldsymbol{\theta} \cdot \mathbf{n}_{\Omega}\right) .
$$

With these results at hand, the abstract framework of Section 4.1 can be readily used to deal with the robust optimization problem (4.2), when small perturbations taking the form of a uniform dilation or erosion are expected on shapes.

As is clear from the statement (and the proof) of Proposition 4.1, these considerations are valid provided the maximum amplitude $m$ of the expected dilations or erosions is small enough so that $\left(\mathrm{Id}+\delta \mathbf{n}_{\Omega}\right)$ is a diffeomorphism, i.e. $\Omega$ and $\Omega_{\delta}$ share the same topology for all $|\delta|<m$. This restriction may impose unrealistically small values on $m$; so as to deal with more concrete situations, we rely on a heuristic adjustment of the above procedure when ( $\left.\mathrm{Id}+\delta \mathbf{n}_{\Omega}\right)$ is not a diffeomorphism. The starting point is the observation that $\left(\mathrm{Id}+\delta \mathbf{n}_{\Omega}\right)$ may fail to be a diffeomorphism because of the existence of points $\mathbf{x} \in \partial \Omega$ such that the segment with endpoints $\mathbf{x}$ and $\mathbf{x}+\delta \mathbf{n}_{\Omega}(\mathbf{x})$ crosses the skeleton (or sometimes also called medial axis) of the shape $\Omega$, which is defined by

$$
\operatorname{Sk}(\Omega):=\left\{\mathbf{x} \in \mathbb{R}^{3}, \exists \mathbf{y}_{1}, \mathbf{y}_{2} \in \partial \Omega, \mathbf{y}_{1} \neq \mathbf{y}_{2} \text { and } d(x, \partial \Omega)=\left|\mathbf{x}-\mathbf{y}_{1}\right|=\left|\mathbf{x}-\mathbf{y}_{2}\right|\right\} .
$$

The skeleton $\operatorname{Sk}(\Omega)$ may alternatively be seen as the set of points $\mathbf{x} \in \mathbb{R}^{3}$ where the squared distance function $d_{\Omega}^{2}$ is not differentiable; see for instance [22] about these points, and Fig. 4 for an illustration.

When this happens, we simply replace $\Omega_{\delta}$ by the modified version

$$
\Omega_{s}:=\left(\operatorname{Id}+s(\mathbf{x}) \mathbf{n}_{\Omega}\right)(\Omega),
$$

where for $\mathbf{x} \in \partial \Omega,|s(\mathbf{x})|<\delta$ is calculated so that so that the segment joining $\mathbf{x}$ to $\mathbf{x}+s(\mathbf{x}) \mathbf{n}_{\Omega}(\mathbf{x})$ does not intersect $\operatorname{Sk}(\Omega)$ - i.e. the considered dilation or erosion of $\Omega$ stops when $\operatorname{Sk}(\Omega)$ is encountered. To achieve this purpose, we proceed as in $[19,50]$, relying on the knowledge of the signed distance function $d_{\Omega}$ : for every point $\mathbf{x}, s(\mathbf{x})$ is the first value $s<0$ (resp. $s>0$ ) such that the function $s \rightarrow d_{\Omega}\left(\mathbf{x}+s \nabla d_{\Omega}(\mathbf{x})\right.$ ) is no longer monotone in the case of an erosion (resp. dilation). 

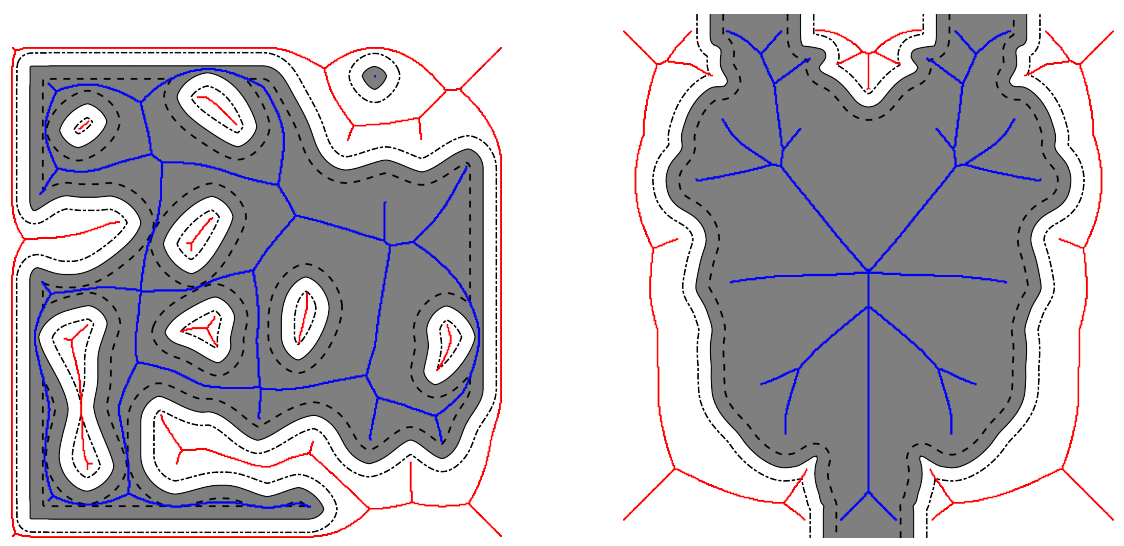

Figure 4 - Two examples of shapes (in grey) with their skeleton. The contours of the dilated and eroded versions of both shapes are represented in dashed lines, and the interior (resp. exterior) part of the skeleton $\operatorname{Sk}(\Omega) \cap \Omega($ resp. $\operatorname{Sk}(\Omega) \cap(\mathcal{D} \backslash \bar{\Omega}))$ appears in blue (resp. red).

Remark 4.3. Depending on the particular etching technology, perturbations $\Omega_{\delta}$ of the optimized shape $\Omega$ may adopt a more complicated structure than that (4.8) featuring a constant parameter $\delta$. For instance, in some situations, $\delta$ could be a function of the depth $y$; see the survey [38].

\subsubsection{Robust shape optimization with respect to defects caused by the lithography process: a description using Gaussian kernels}

In computational lithography, it is commonly accepted [30] that manufacturing a "blueprint", ( $y$-invariant) admissible shape

$$
\Omega=\left\{(x, y, z) \in \mathbb{R}^{3},(x, z) \in \widehat{\Omega}, y \in(0, h)\right\}
$$

will result effectively in a smeared version $\Omega_{\delta} \subset \mathbb{R}^{3}$ given by:

$$
\Omega_{\delta}=\left\{(x, y, z) \in \mathbb{R}^{3},\left(\chi_{\widehat{\Omega}} * \mathcal{G}_{\delta}\right)(x, z)>\frac{1}{2}, y \in(0, h)\right\},
$$

where $\chi_{\widehat{\Omega}}$ is the characteristic function of the two-dimensional section $\widehat{\Omega} \subset \widehat{\mathcal{D}_{\text {opt }}}$, and $\mathcal{G}_{\delta}(\boldsymbol{\xi})$ is the Gaussian kernel with mean 0 and standard deviation $\delta$ :

$$
\forall \boldsymbol{\xi} \in \mathbb{R}^{2}, \mathcal{G}_{\delta}(\boldsymbol{\xi})=\frac{1}{2 \pi \delta^{2}} e^{-\frac{|\boldsymbol{\xi}|^{2}}{2 \delta^{2}}}
$$

Intuitively, perturbations of the form (4.10) imply that, if for instance the boundary $\partial \Omega$ is flat, $\partial \Omega_{\delta}$ coincides with $\partial \Omega$; however, if it has positive or negative curvature, the sharp feature of $\partial \Omega$ is smeared; see Fig. 5 for a two-dimensional illustration. In general, $\Omega_{\delta}$ depends on global features of $\Omega$, but it is mostly influenced by the curvature of $\partial \Omega$, in a rather non explicit fashion. 


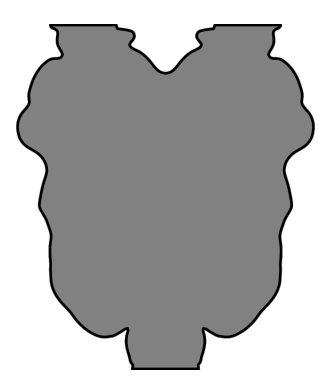

(a) Original shape $\Omega$

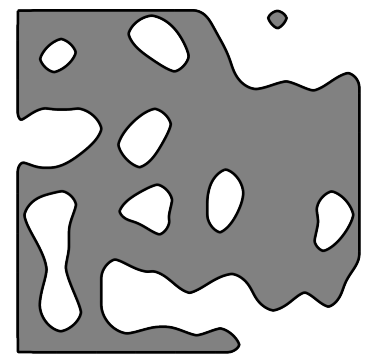

(d) Original shape $\Omega$

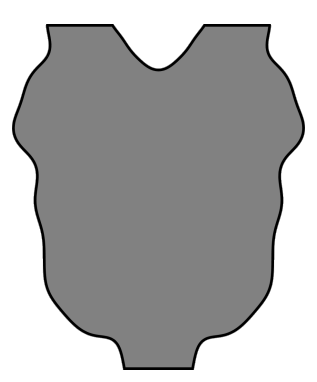

(b) Perturbed shape $\Omega_{\delta}$

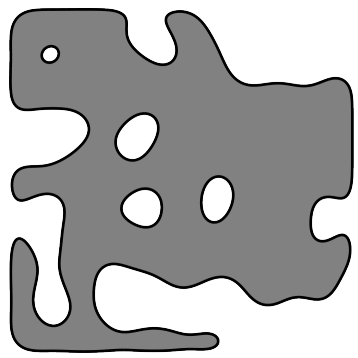

(e) Perturbed shape $\Omega_{\delta}$

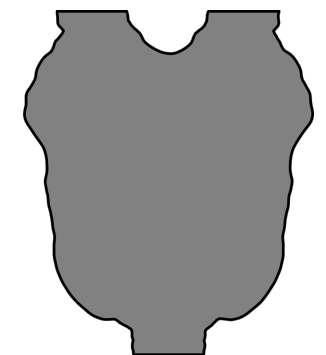

(c) Local approximation $\widetilde{\Omega_{\delta}}$

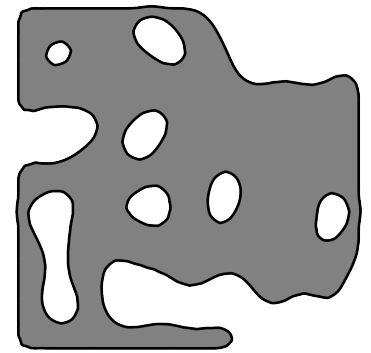

(f) Local approximation $\widetilde{\Omega_{\delta}}$

Figure 5 - Comparison between a shape $\Omega$, its perturbed version $\Omega_{\delta}$ given by (4.10), and the approximation $\widetilde{\Omega_{\delta}}$ defined by (4.12).

The robust optimization problem (4.3) with respect to uncertainties caused by lithography then involves the perturbed functional $J_{\delta}(\Omega):=J\left(\Omega_{\delta}\right)$. The study of $J_{\delta}(\Omega)$, and notably its shape derivative, is quite intricate because of the dependence of $\Omega_{\delta}$ on global features of $\Omega$. To alleviate this difficulty we trade $\Omega_{\delta}$ for an approximate counterpart $\widetilde{\Omega_{\delta}}$ of the form:

$$
\widetilde{\Omega_{\delta}}=\left(\operatorname{Id}+p_{\Omega, \delta} \mathbf{n}_{\Omega}\right)(\Omega),
$$

for a scalar field $p_{\Omega, \delta}: \partial \Omega \rightarrow \mathbb{R}$, whose definition we now describe in detail.

For an arbitrary, given point $\mathbf{x}_{0}=\left(x_{0}, y_{0}, z_{0}\right) \in \partial \Omega$ with projection $\widehat{\mathbf{x}}_{0}:=\left(x_{0}, z_{0}\right)$ onto $\widehat{\Omega}$, we consider the local, second-order approximation of the section $\widehat{\Omega}$ near $\widehat{\mathbf{x}}_{0}$, by means of the half-space $\mathcal{P}_{\Omega, \mathbf{x}_{0}}$ defined by (see Fig. 6):

$$
\mathcal{P}_{\Omega, \mathbf{x}_{0}}=\left\{\widehat{\mathbf{x}}_{0}+\mathbf{z} \in \mathbb{R}^{2}, z_{n}<\kappa\left(\widehat{\mathbf{x}}_{0}\right) z_{\tau}^{2}\right\}
$$

In the latter formula, we have denoted by $z_{n}:=\mathbf{z} \cdot \mathbf{n}_{\widehat{\Omega}}\left(\widehat{\mathbf{x}}_{0}\right)$ and $z_{\tau}:=\mathbf{z} \cdot \boldsymbol{\tau} \widehat{\Omega}_{\Omega}\left(\widehat{\mathbf{x}}_{0}\right)$, the normal and tangential components of a vector $\mathbf{z} \in \mathbb{R}^{2}$ in the local frame $\left(\boldsymbol{\tau} \widehat{\Omega}\left(\widehat{\mathbf{x}}_{0}\right), \mathbf{n}_{\widehat{\Omega}}\left(\widehat{\mathbf{x}}_{0}\right)\right)$ at $\widehat{\mathbf{x}}_{0}$ obtained by gathering the tangent $\boldsymbol{\tau} \widehat{\Omega}\left(\widehat{\mathbf{x}}_{0}\right)$ and the normal vector $\mathbf{n}_{\widehat{\Omega}}\left(\widehat{\mathbf{x}}_{0}\right)$ to $\widehat{\Omega}$ at $\widehat{\mathbf{x}}_{0}$. Finally, $\kappa\left(\widehat{\mathbf{x}}_{0}\right)$ is the mean curvature of $\widehat{\Omega}$ at $\widehat{\mathbf{x}}_{0}$.

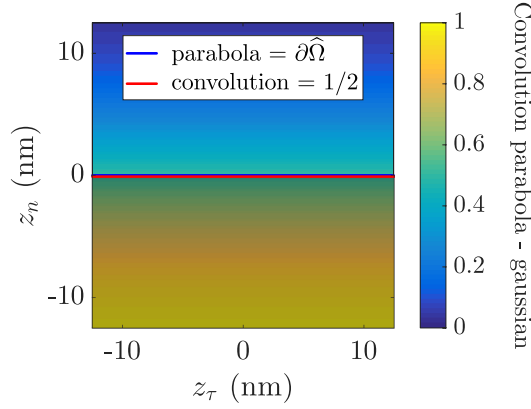

(a) Curvature $\kappa=0$

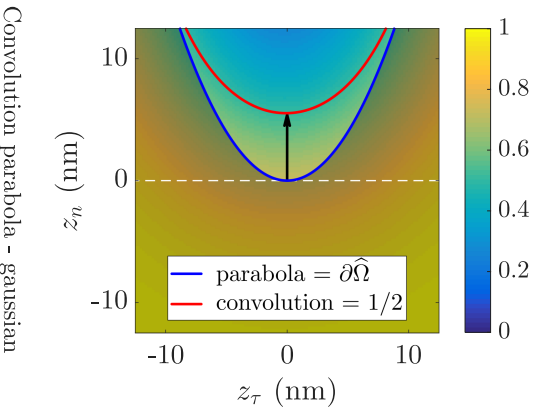

(b) Curvature $\kappa>0$

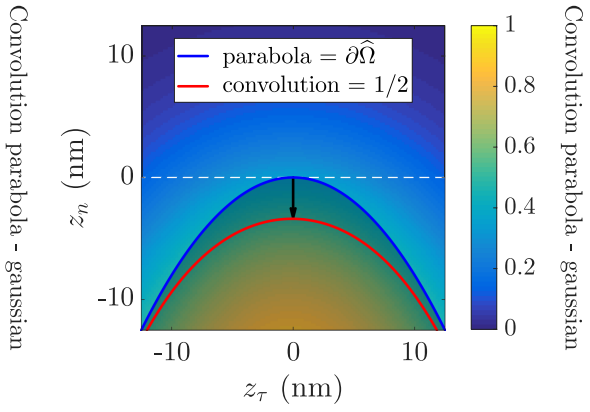

(c) Curvature $\kappa<0$

Figure 6 - Schematic representation of the mapping $p_{\Omega, \delta}$ involved in the definition (4.12) of the approximate perturbation $\widetilde{\Omega_{\delta}}$. 
For $\widehat{\mathbf{x}}=(x, z) \in \mathbb{R}^{2}$ close to $\widehat{\mathbf{x}}_{0}$, taking advantage of the smallness of $\delta$, we then have:

$$
\left(\chi_{\widehat{\Omega}} * \mathcal{G}_{\delta}\right)(x, z)=\int_{\mathbb{R}^{2}} \chi_{\widehat{\Omega}}(\widehat{\mathbf{y}}) \mathcal{G}_{\delta}(\widehat{\mathbf{x}}-\widehat{\mathbf{y}}) d \widehat{\mathbf{y}} \approx F_{\Omega, \mathbf{x}_{0}}(\widehat{\mathbf{x}}),
$$

where

$$
F_{\Omega, \mathbf{x}_{0}}(\widehat{\mathbf{x}}):=\int_{\mathbb{R}^{2}} \chi_{\mathcal{P}_{\Omega, \mathbf{x}_{0}}}(\widehat{\mathbf{y}}) \mathcal{G}_{\delta}(\widehat{\mathbf{x}}-\widehat{\mathbf{y}}) d \widehat{\mathbf{y}}
$$

is the convolution between the characteristic function of the local second-order approximation of $\partial \widehat{\Omega}$ at $\widehat{\mathbf{x}}_{0}$ and the Gaussian kernel (4.11). We then define $p_{\Omega, \delta}\left(\mathbf{x}_{0}\right)$ as the unique value $s \in \mathbb{R}$ such that

$$
f(s):=F_{\Omega, \mathbf{x}_{0}}\left(\widehat{\mathbf{x}}_{0}+s \mathbf{n}_{\widehat{\Omega}}\left(\widehat{\mathbf{x}}_{0}\right)\right)=\frac{1}{2},
$$

which makes sense since

$$
f(s)=\int_{\mathbb{R}} \int_{-\infty}^{\kappa\left(\mathbf{x}_{0}\right) z_{\tau}^{2}-s} \mathcal{G}_{\delta}\left(z_{\tau} \boldsymbol{\tau}_{\widehat{\Omega}}\left(\widehat{\mathbf{x}}_{0}\right)+t \mathbf{n}_{\widehat{\Omega}}\left(\widehat{\mathbf{x}}_{0}\right)\right) d t d z_{\tau}=\frac{1}{2 \sqrt{2 \pi} \delta} \int_{\mathbb{R}} e^{-\frac{x^{2}}{2 \delta^{2}}} \operatorname{erfc}\left(\frac{s-\kappa\left(\widehat{\mathbf{x}}_{0}\right) x^{2}}{\sqrt{2} \delta}\right) d x
$$

(where $\operatorname{erfc}(x)=2 / \sqrt{\pi} \int_{x}^{\infty} \exp \left(-t^{2}\right) d t$ refers to the so-called complementary error function) is a smooth, decreasing function with

$$
\lim _{s \rightarrow-\infty} f(s)=1 \text { and } \lim _{s \rightarrow+\infty} f(s)=0 .
$$

Notice that $p_{\Omega, \delta}\left(\mathbf{x}_{0}\right)$ only depends on $\widehat{\Omega}$ via its curvature at $\widehat{\mathbf{x}}_{0}$ however the dependence is not explicit. Nevertheless, $p_{\Omega, \delta}$ is easy to calculate numerically. As is exemplified on Fig. 5 this approximation performs reasonably well: the approximate perturbation $\widetilde{\Omega_{\delta}}$ is close to $\Omega_{\delta}$, except that it fails to capture the topological changes between $\Omega_{\delta}$ and $\Omega$.

Returning to our robust optimization problem, the implementation of (4.3) relies on the shape derivative of the perturbated functional

$$
\widetilde{J}_{\delta}(\Omega):=J\left(\left(\operatorname{Id}+p_{\Omega, \delta} \mathbf{n}_{\Omega}\right)(\Omega)\right) .
$$

The rigorous calculation and the practical use of this shape derivative are not simple since $p_{\Omega, \delta}$ brings into play the curvature of the interface $\partial \Omega$. To simplify this calculation, we simply neglect the dependence of $p_{\Omega, \delta}$ on $\Omega$, so that the shape derivative $J_{\delta}^{\prime}(\Omega)$ is simply given by (4.9) where $\delta$ is replaced by $p_{\Omega, \delta}$. Although simplistic, this approximation gives pretty good results as presented in Section 6.4.4 and it has the advantage of being simple and fast to implement.

\section{$5 \quad$ Numerical representation of shapes and general algorithm}

\subsection{Level set representation}

When it comes to the numerical representation of shapes and their evolution, we rely on the level set method; see [55] for the pioneering work and $[6,63,71]$ for the introduction of this method in the shape optimization context. In a nutshell, a generic shape $\Omega \subset \mathcal{D}_{\text {opt }}$ is represented as the negative subdomain of a scalar "level set" function $\phi: \mathcal{D}_{\mathrm{opt}} \rightarrow \mathbb{R}$, i.e.

$$
\forall \mathbf{x} \in \mathcal{D}_{\text {opt }}, \begin{cases}\phi(\mathbf{x})<0 & \text { if } \mathbf{x} \in \Omega, \\ \phi(\mathbf{x})=0 & \text { if } \mathbf{x} \in \partial \Omega \backslash \partial \mathcal{D}_{\text {opt }}, \\ \phi(\mathbf{x})>0 & \text { if } \mathbf{x} \in \mathcal{D}_{\text {opt }} \backslash \bar{\Omega} .\end{cases}
$$

In this context, if $\Omega \equiv \Omega(t)$ is a shape evolving over a time period $(0, T)$ according to a velocity field with normal component $v(t, \mathbf{x})$, this motion translates in terms of an associated level set function $\phi(t, \cdot)$ (i.e. (5.1) holds at each time $t \in(0, T))$ into the following Hamilton-Jacobi equation:

$$
\frac{\partial \phi}{\partial t}(t, \mathbf{x})+v(t, \mathbf{x})|\nabla \phi(t, \mathbf{x})|=0, t \in(0, T), \mathbf{x} \in \mathcal{D}_{\text {opt }},
$$

an equation for which there exist efficient numerical solvers; see for instance [62, Section 6.4], [51]. 
In our applications, the normal velocity $v(t, \mathbf{x})$ is the shape gradient of the considered objective functional $J(\Omega)$, obtained from its shape derivative via the solution of (3.10). Since the considered shapes are invariant in the $y$ direction (see Section 3.1 and (3.2)), the numerical resolution of (5.2) is actually performed in two dimensions, in a computational domain accounting for any horizontal section

$$
\widehat{\mathcal{D}_{\mathrm{opt}}}=\left\{\mathbf{x}=(x, y, z) \in \mathcal{D}_{\mathrm{opt}}, y=\mathrm{cst}\right\}
$$

of the design domain $\mathcal{D}_{\text {opt }}$.

Remark 5.1. In practice, for numerical stability reasons, the level set function $\phi(t, \cdot)$ is periodically reinitialized so that it coincides with the signed distance function to the evolving shape $\Omega(t)$, at least in a neighborhood of $\partial \Omega(t)[16]$.

\subsection{Implementation and algorithm}

Our numerical algorithm is composed of several building blocks which are implemented either in Matlab ${ }^{\circledR}$ [68] or in COMSOL Multiphysics ${ }^{\circledR}[17]$.

The optimized shape $\Omega$ is represented by means of a level set function $\phi$ defined at the vertices of a Cartesian grid $\mathcal{G}$ of the two-dimensional section $\widehat{\mathcal{D}_{\text {opt }}}$ of the design domain; see Section 5.1 and Remark 5.1. The resolution of the Hamilton-Jacobi equation (5.2) and the reinitialization of the level set function $\phi$ - see Remark 5.1 - rely on an ENO scheme from the ToolboxLS [51] toolbox.

The three-dimensional resolution of the Maxwell system (2.25) is carried out on a tetrahedral mesh $\mathcal{T}$ of the computational domain $\mathcal{D}$; it relies on the finite element method using second-order Nédélec vector elements [40, Section 8] [53, Section 5.5] implemented in COMSOL Multiphysics ${ }^{\circledR}$ [17]. Since the nodes of the grid $\mathcal{G}$ do not necessarily coincide with the vertices of the tetrahedral mesh $\mathcal{T}$, we consistently rely on interpolation operations between these two supports.

The optimization algorithm combining these features is driven by a Matlab ${ }^{\circledR}$ [68] implementation. A version is sketched in Algorithm 1, which is dedicated to the maximization of the minimum value between $N_{\text {obj }}$ objective functions $J_{i}(\Omega), i=1, \ldots, N_{\text {obj }}$, using $N_{\text {in }}$ inputs (associated to different perturbations of the actual shape or injection boundary conditions).

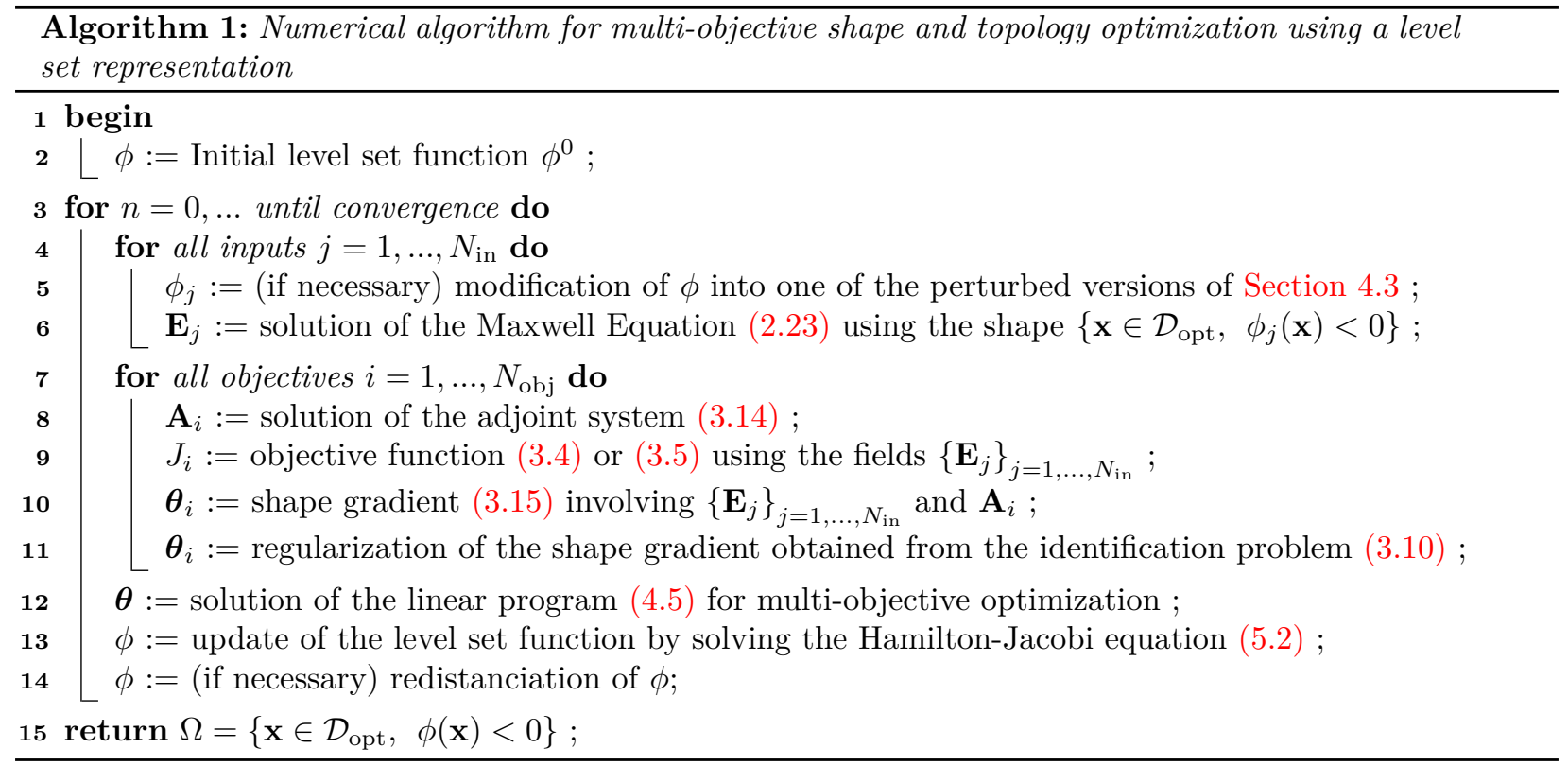

Eventually, as far as the stopping criterion is concerned, we have simply used a fixed number of iterations of the above procedure in all the examples considered in Section 6.

\section{$6 \quad$ Numerical examples}

In this section, we evaluate the efficiency of our shape and topology optimization algorithm on the design of various nanophotonic devices. 
In all cases, the design domain $\mathcal{D}_{\text {opt }}$ is $\left[-d_{x}, d_{x}\right] \times[-h / 2, h / 2] \times\left[-d_{z}, d_{z}\right]$ where $d_{x}$ and $d_{z}$ vary between 1.5 to $3 \mu \mathrm{m}$ depending on the situation, and $h=306 \mathrm{~nm}$. The computational domain $\mathcal{D}$ is then

$\mathcal{D}=\left[-d_{x}-l-w_{\mathrm{PML}}, d_{x}+l+w_{\mathrm{PML}}\right] \times\left[-(h+l) / 2-w_{\mathrm{PML}},(h+l) / 2+w_{\mathrm{PML}}\right] \times\left[-d_{z}-l-w_{\mathrm{PML}}, d_{z}+l+w_{\mathrm{PML}}\right]$,

where $l=1 \mu \mathrm{m}$, and the thickness of the perfectly matched layer (see Section 2.4.2) is $w_{\mathrm{PML}}=500 \mathrm{~nm}$. The tetrahedral mesh $\mathcal{T}$ associated to the finite element resolution of (2.23) is composed of about $10^{5}$ elements with size at most $\lambda /(5 n)$ - as is commonly advised in the practice of such simulations. The $2 \mathrm{~d}$ Cartesian grid $\mathcal{G}$ of $\widehat{\mathcal{D}_{\text {opt }}}$ dedicated to practice of the level set method has uniform size $\Delta x=10 \mathrm{~nm}$.

The values of the wavelength $\lambda$ considered in this article lie within the typical range used in telecommunications, that is around $1.31 \mu \mathrm{m}$ and $1.55 \mu \mathrm{m}$. The values of the refractive indices of the materials at stake are:

$$
n_{\text {core }} \approx 3.49, n_{\text {subs }} \approx 1.44, \text { and } n_{\text {clad }}=1 \text {. }
$$

Our numerical examples fall into two categories: at first, in Sections 6.1 and 6.2, the validity of the shape optimization framework of Sections 3 and 5 is evaluated on fairly classical optimization test case of nanophotonic devices. The subsequent Sections 6.3 and 6.4 deal more specifically with robustness issues; in there, we show how the strategy of Section 4 makes it possible to obtain devices that are robust to both wavelength uncertainties and uncertainties caused by the lithography and etching manufacturing processes.

All the numerical computations are performed on a cluster node with 8 to 20 cores CPU clocked at 3.0 $\mathrm{GHz}$ with $128 \mathrm{~GB}$ of reserved memory. For each example, we provide a rough estimate of the needed CPU time; notice that in each case, more than $99 \%$ of this time is devoted to the resolution of the state or adjoint Maxwell equations (2.25) and (3.14); the effort related to the level set method is negligible by comparison.

\subsection{Optimization examples involving a single objective function}

\subsubsection{Optimization of the shape of a crossing device}

Our first numerical example deals with the optimization of the shape of a crossing component, whose purpose is to limit the coupling losses between two adjacent waveguides with different orientations (see Fig. 7). The design domain $\mathcal{D}_{\text {opt }}$ is connected to two input waveguides via the ports $\Gamma_{\mathrm{in}, 1}$ and $\Gamma_{\mathrm{in}, 2}$, and two outgoing waveguides via the surfaces $\Gamma_{\text {out }, 1}$ and $\Gamma_{\text {out,2 }}$. The fundamental mode $\mathrm{TE}_{0}$ is injected at $\Gamma_{\mathrm{in}, 1}\left(\operatorname{resp} . \Gamma_{\mathrm{int}, 2}\right)$ with a wavelength $\lambda=1.55 \mu \mathrm{m}$ and our aim is to maximize the transmitted energy $J_{\mathrm{o}, 1}(\Omega)$ to the fundamental mode in $\Gamma_{\text {obj }}=\Gamma_{\text {out }, 1}\left(\right.$ resp. $\left.\Gamma_{\text {out }, 2}\right)$.

Taking advantage of the symmetry of the situation, we only consider shapes $\Omega$ which are symmetric with respect to the $x$ and $z$ axes; doing so ensures the symmetry between the electromagnetic fields $\mathbf{E}_{\Omega}$ and $\mathbf{H}_{\Omega}$ obtained in the situations where light is injected from $\Gamma_{\mathrm{in}, 1}$ and $\Gamma_{\mathrm{in}, 2}$. Hence, our shape optimization problem boils down to that of maximizing the single objective

$$
\max _{\Omega} J_{\mathrm{o}, 1}(\Omega),
$$

where the Maxwell equations (2.23) describing the physics at play involve only injection through the port $\Gamma_{\mathrm{in}, 1}$ and where $\Gamma_{\mathrm{obj}}=\Gamma_{\mathrm{out}, 1}$. Accordingly, in the practice of the boundary variation method of Hadamard, we only consider symmetrized vector fields $\boldsymbol{\theta}$ of the form

$$
\forall(x, z) \in \widehat{\mathcal{D}_{\mathrm{opt}}}, \tilde{\boldsymbol{\theta}}(x, z)=\frac{1}{4}(\boldsymbol{\theta}(x, z)+\boldsymbol{\theta}(-x, z)+\boldsymbol{\theta}(x,-z)+\boldsymbol{\theta}(-x,-z)) .
$$



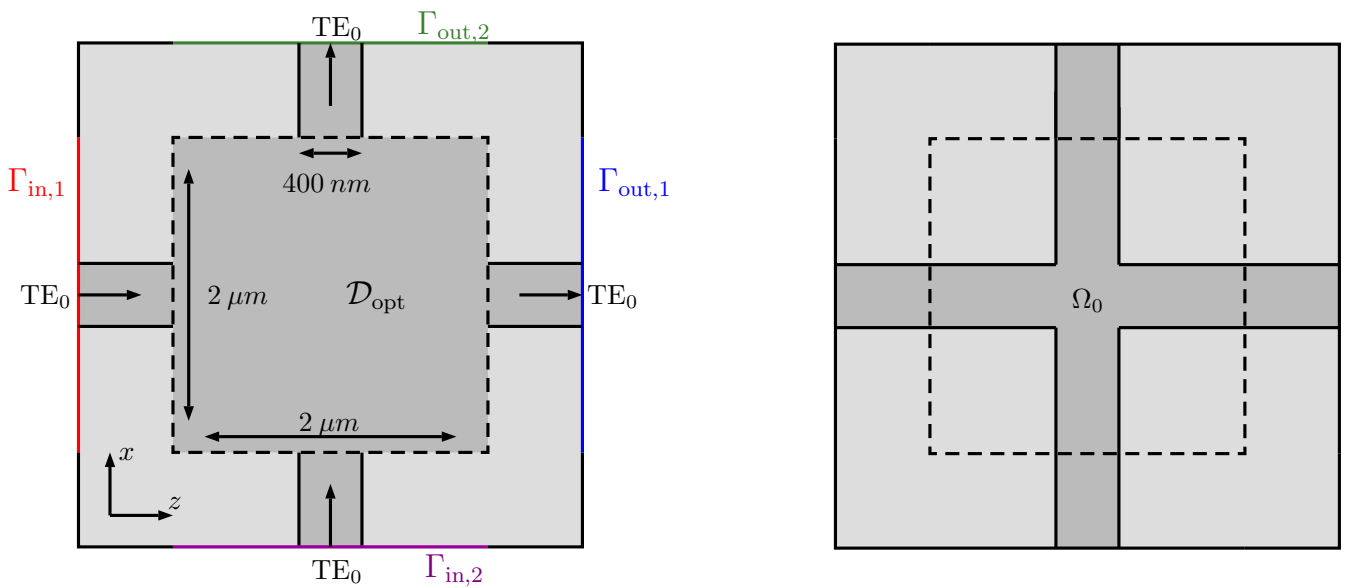

Figure 7 - (Left) Setting of the crossing optimization test-case of Section 6.1.1; (right) Initial design.

Starting from an initial shape made of the reunion of two orthogonal, straight waveguides connecting $\Gamma_{\text {in }, 1}$ to $\Gamma_{\text {out }, 1}$ and $\Gamma_{\text {in }, 2}$ to $\Gamma_{\text {out, } 2}$ (see Fig. 7 (right)), 50 iterations of our optimization algorithm are performed, for a total computational time of roughly 4 hours. The optimized design, convergence history, as well as the normalized density of electromagnetic energy

$$
\mathcal{E}_{\Omega}(\mathbf{x}):=\varepsilon\left|\mathbf{E}_{\Omega}(\mathbf{x})\right|^{2}+\mu\left|\mathbf{H}_{\Omega}(\mathbf{x})\right|^{2}, \mathbf{x} \in \mathcal{D}
$$

stored inside the computational domain are represented on Fig. 8. We notice that more than $95 \%$ of the electromagnetic energy contained in the incoming field is successfully conveyed to the output ports, while this ratio equals only $70 \%$ in the case of the initial device of Fig. 7 (right). The shape $\Omega$ has changed topology in the course of the optimization process, which is a well-known benefit from the use of the level set method for describing the motion of shapes.

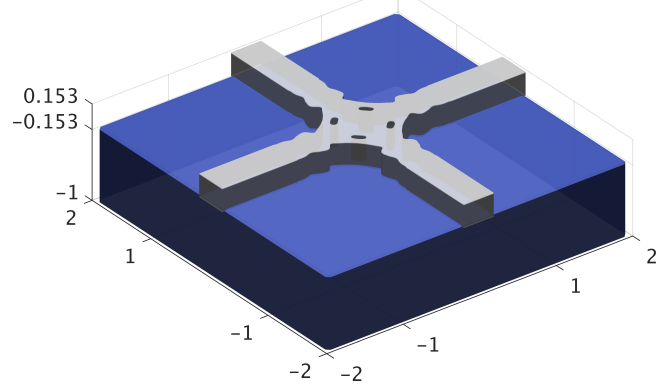

(a) Optimized shape (axes in $\mu m$ )

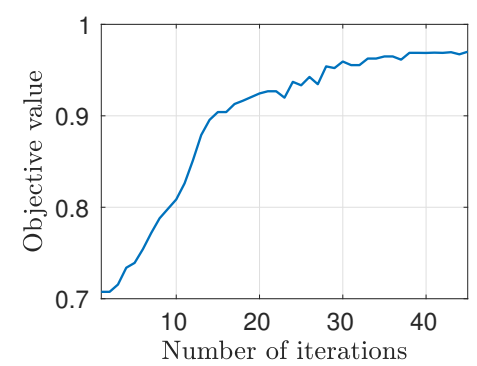

(b) Convergence history

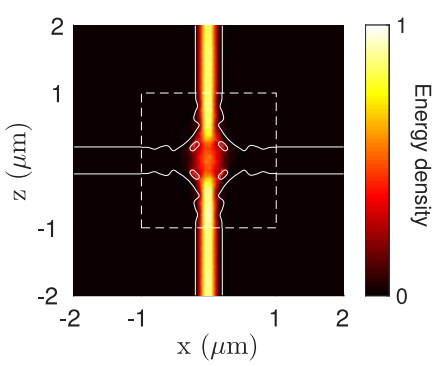

(c) $\mathcal{E}_{\Omega}$ in the section $\{y=0\}$

Figure 8-Optimized shape of the crossing device of Section 6.1.1 and details of the numerical computation.

\subsubsection{Optimization of the shape of a mode converter}

Our second example deals with the optimization of the shape of a device whose purpose is to transform the mode coming from an input waveguide into another mode of the output waveguide. The physical setting is depicted on Fig. 9, where the output waveguide is wide enough to allow for the existence of multiple guided modes. In this context, the electromagnetic power is injected via the port $\Gamma_{\mathrm{in}}$, using the fundamental mode $\mathrm{TE}_{0}$, and we seek to transfer this power to the $3^{\mathrm{rd}}$ mode $\mathrm{TE}_{2}$ of the output waveguide; in other terms, we solve the problem

$$
\max _{\Omega} J_{\mathrm{o}, 3}(\Omega),
$$

in which the objective surface is $\Gamma_{\mathrm{obj}}=\Gamma_{\mathrm{out}}$. Due to symmetry of the situation with respect to the $x$ variable, only one half of the design domain $\mathcal{D}_{\text {opt }}$ is discretized and we use symmetrized deformation fields of the form

$$
\forall(x, z) \in \widehat{\mathcal{D}_{\mathrm{opt}}}, \quad \tilde{\boldsymbol{\theta}}(x, z)=\frac{1}{2}(\boldsymbol{\theta}(x, z)+\boldsymbol{\theta}(-x, z))
$$


in the practice of Hadamard's method.
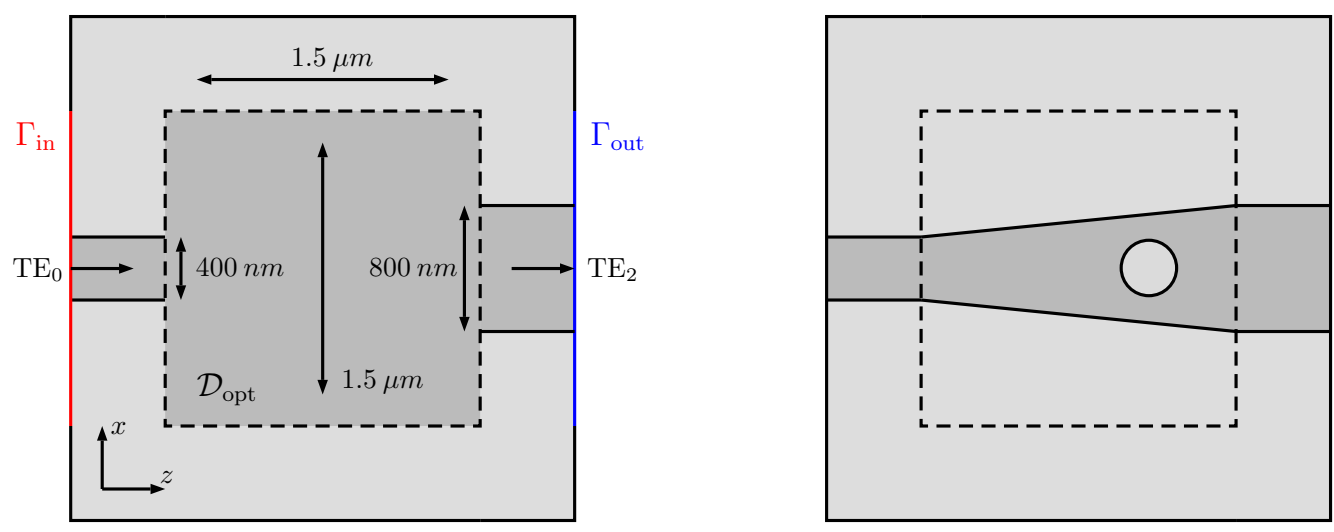

Figure 9 - (Left) Setting of the mode converter optimization test-case of Section 6.1.2; (right) initial design .

Starting from the initial shape in Fig. 9 (right), 50 iterations of our shape optimization algorithm are performed, for a total CPU time of approximately 4 hours; the result is displayed on Fig. 10. The optimized design allows to redirect more than $85 \%$ of the incoming power via the fundamental mode $\mathrm{TE}_{0}$ of the input waveguide into the third mode $\mathrm{TE}_{2}$ of the output waveguide.

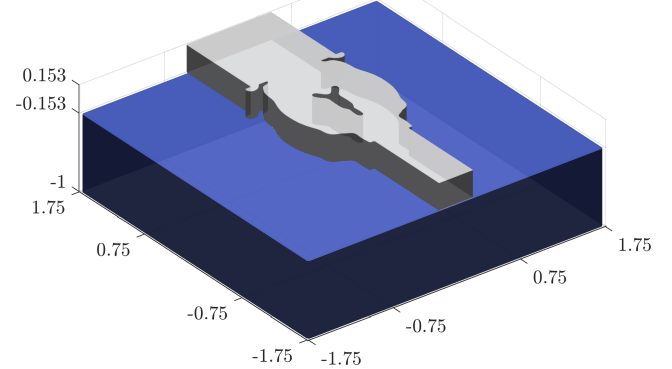

(a) Optimized shape (axes in $\mu \mathrm{m}$ )

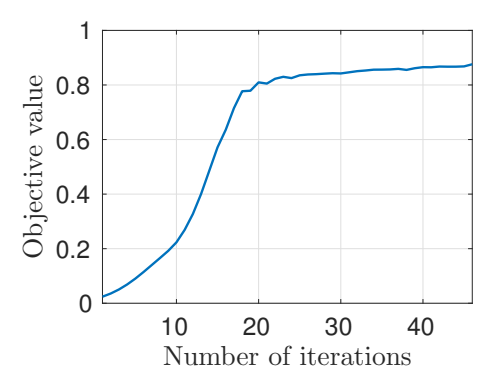

(b) Convergence history

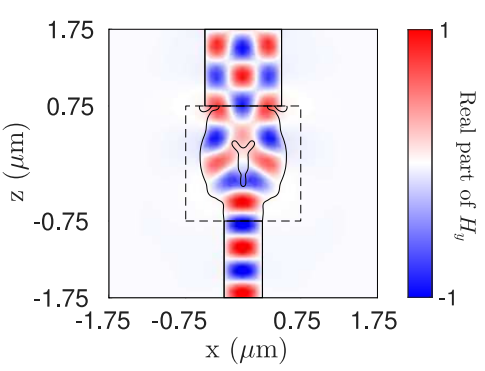

(c) Real part of $H_{y}$ in $\{y=0\}$

Figure 10 - Optimized shape of the mode converter of Section 6.1.2 and details of the numerical computation.

\subsubsection{Shape optimization of a power divider}

Let us now turn to the optimization of another very useful device in nanophotonics, namely the power divider. The physical setting is that of Fig. 11: our aim is to divide the electromagnetic power conveyed by the incoming field through the waveguide figured by $\Gamma_{\text {in }}$ in an equal way between both output waveguides $\Gamma_{\text {out }, 1}$ and $\Gamma_{\text {out }, 2}$. As in the previous example, the symmetry of the problem allows to discretize only one half of the design domain $\mathcal{D}_{\text {opt }}$ and to restrict the set of the considered deformation fields $\boldsymbol{\theta}$ considered in the practice of Hadamard's method to vector fields of the form (6.5). This allows to formulate our optimization problem as

$$
\max _{\Omega} J_{\mathrm{o}, 1}(\Omega),
$$

that (6.2) in which the objective surface $\Gamma_{\text {obj }}$ is $\Gamma_{\text {out }, 1}$. 

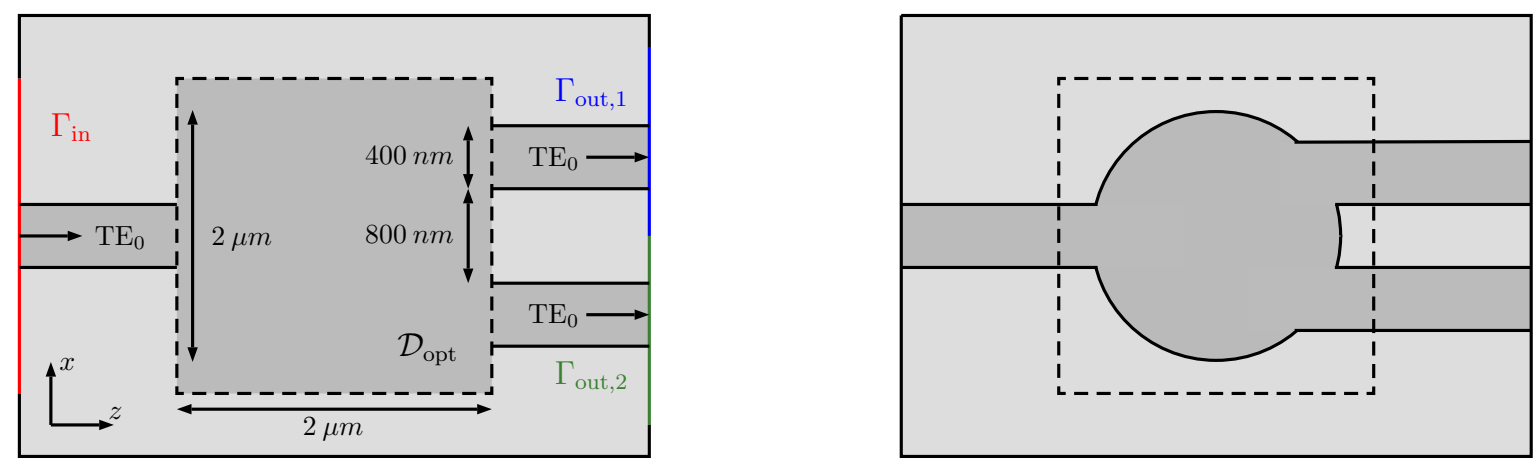

Figure 11 - Setting of the power divider optimization problem of Section 6.1.3.

Starting from the initial shape of Fig. 11 (right), 50 iterations of our optimization algorithm are performed, for a total computational time of roughly 3 hours. Details of the numerical computation are reported on Fig. 12; the optimized device achieves approximately $49 \%$ transmission into each output waveguides.

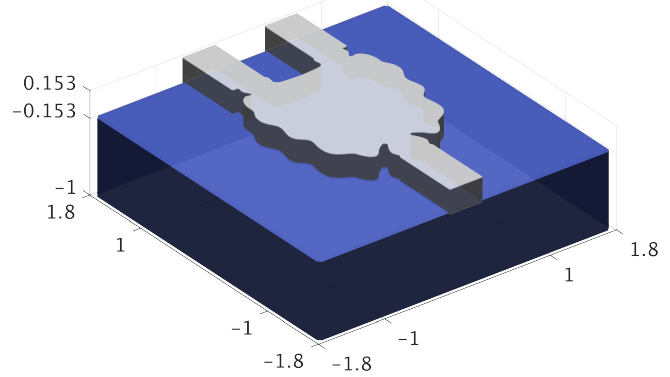

(a) Optimized shape (axes in $\mu m$ )

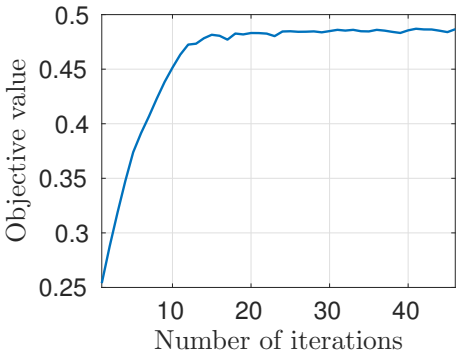

(b) Convergence history

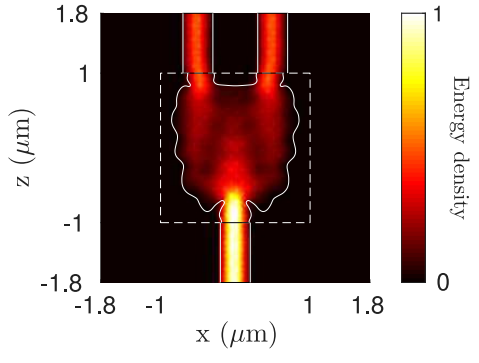

(c) $\mathcal{E}_{\Omega}$ in the section $\{y=0\}$

Figure 12 - Optimized shape of the power divider of Section 6.1 .3 and details of the numerical computation.

\subsubsection{Optimization of the shape of a polarization converter}

Let us finally consider a slightly more involved device, namely a polarization converter. In the physical situation of Fig. 13, our goal is to convert an injected Transverse Electric mode (whose electromagnetic energy is mostly concentrated in the electric field E) into a Transverse Magnetic output mode, which concentrates most of its energy in the magnetic field $\mathbf{H}$. More precisely, we solve the optimization problem

$$
\max _{\Omega} J_{\mathrm{o}, 2}(\Omega)
$$

in which the objective surface $\Gamma_{\text {obj }}$ is $\Gamma_{\text {out }}$, where the first guided mode of the incoming waveguide is TE and is injected through $\Gamma_{\text {in }}$, and where the second guided mode of the outgoing waveguide is TM. 


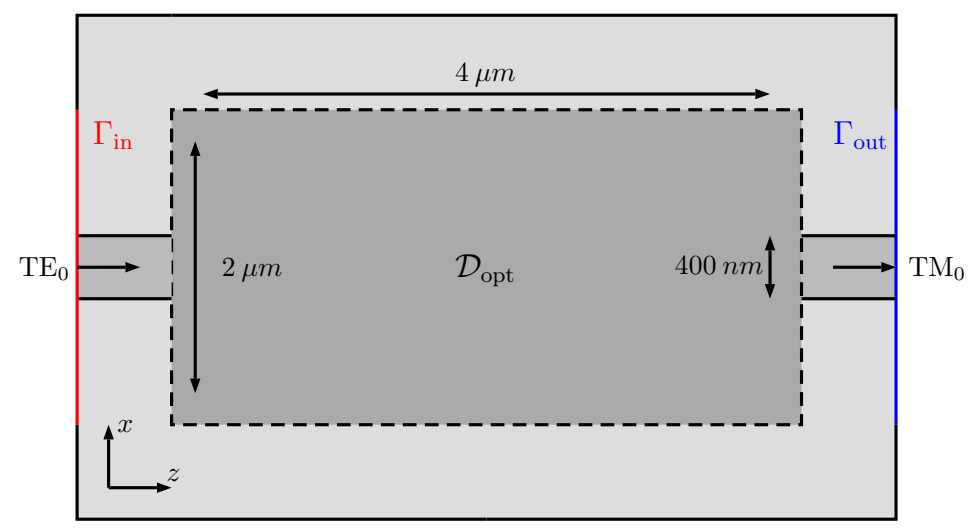

Figure 13 - Schematic representation of the polarization converter optimization problem of Section 6.1.4.

In this situation, there is no natural initial shape, and so we initiate the optimization process with a shape containing multiple regularly spaced holes. At first, we consider an initial shape perforated with $4 \times 2$ holes, and we perform 200 iterations of our algorithm. The results are presented on Fig. 14. The optimized shape has a poor performance since it is only able to transmit $\sim 25 \%$ of the incoming power to the TM mode of the output waveguide.

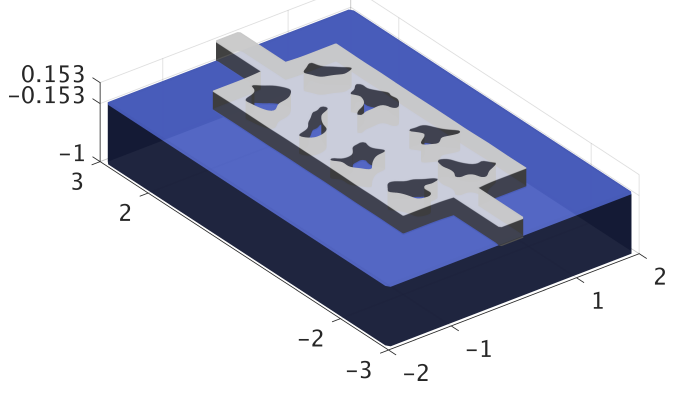

(a) Optimized shape (axes in $\mu m$ )

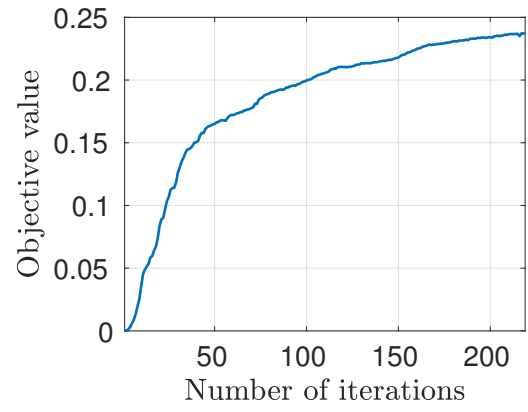

(b) Convergence history

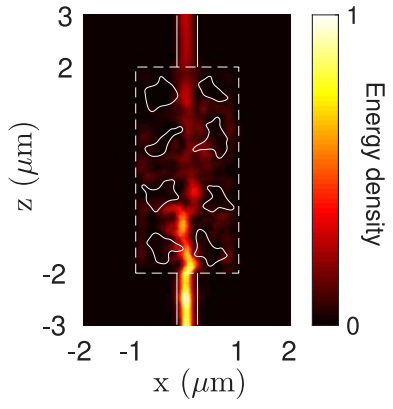

(c) Simulation at $\lambda=1.55 \mu \mathrm{m}$

Figure 14 - Optimized shape of the polarization converter of Section 6.1.4 starting from a design with $4 \times 2$ holes.

Using then an initial shape perforated with $16 \times 8$ holes yields much better results: after roughly 500 iterations of our algorithm, the resulting optimized shape achieves a conversion of about $73 \%$ of the incoming energy into the desired output mode (Fig. 15).

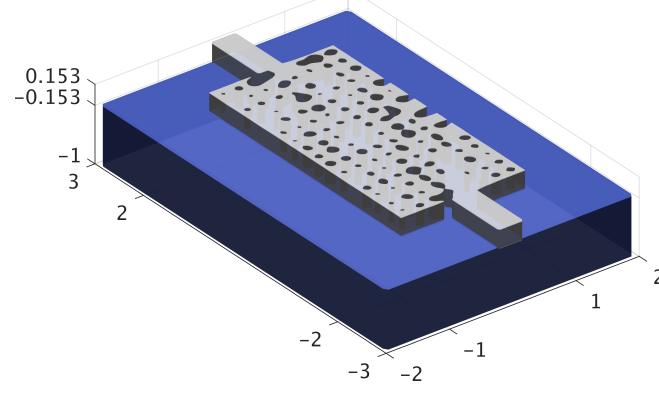

(a) Optimized shape (axes in $\mu m$ )

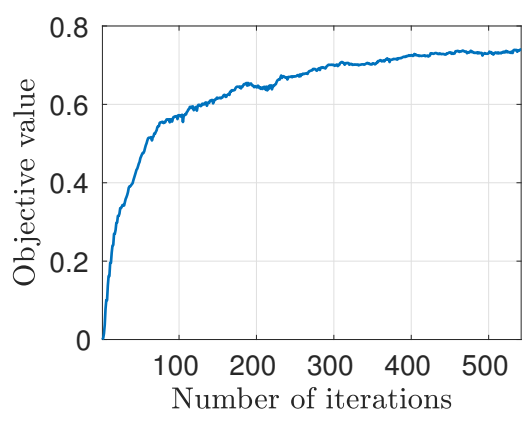

(b) Convergence history

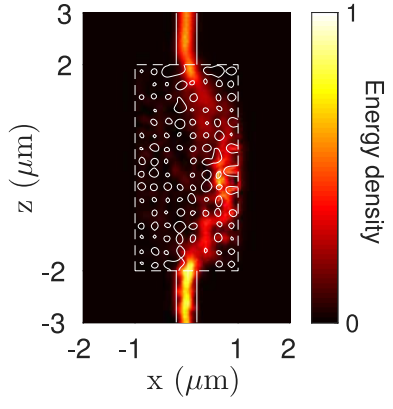

(c) Simulation at $\lambda=1.55 \mu \mathrm{m}$

Figure 15 - Optimized shape of the polarization converter of Section 6.1 .4 starting from a design with $16 \times 8$ holes.

As can be seen in this example, a topology with more holes (showing some sort of microstructures inside the component) tends to enjoy better performance. This behavior is quite customary in shape opti- 
mization problems, where typically no globally optimal design exist, but only multiple locally optimal ones (a refinement of the mesh resolution and an increasingly high number of holes then tend to achieve better performance).

\subsection{Optimization examples involving multiple objective functions}

In this section we discuss the optimization of nanophotonic components in situations which involve the joint maximization of several objective functions. To this end, we rely on the multi-objective methodology described in Section 4.1. In such cases, there is, most of the time, no known "good" initial shape, and we initialize the optimization process with shapes perforated by arrays of holes.

\subsubsection{Optimization of the shape of a duplexer}

Our first example concerns the optimization of the shape of a duplexer, as depicted on Fig. 16. This device aims at redirecting the light injected through the input boundary $\Gamma_{\text {in }}$ into one of the two output waveguides $\Gamma_{\text {out }, 1}, \Gamma_{\text {out }, 2}$ depending on the value of the incident wavelength.

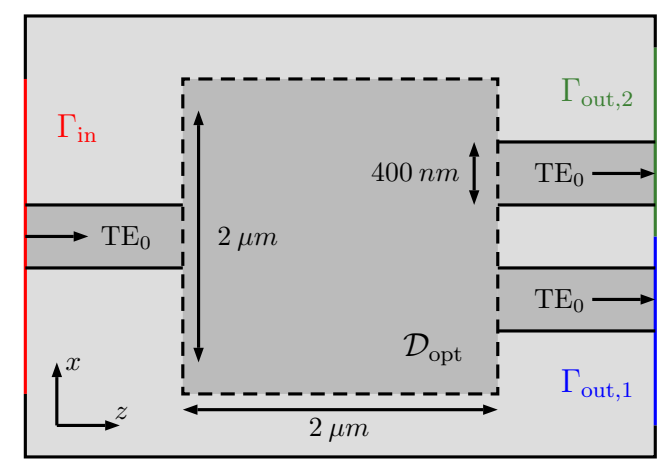

Figure 16 - Schematic representation of the duplexer shape optimization problem of Section 6.2.1.

In this context, the considered shape optimization problem reads:

$$
\max _{\Omega} \min _{i=1,2} J_{i}(\Omega),
$$

in which $J_{i}(\Omega)$ is the version of $J_{\mathrm{o}, 1}(\Omega)$ as defined in (3.5), involving the objective surface $\Gamma_{\mathrm{obj}}=\Gamma_{\text {out }, i}$, in the situation where the operating wavelength equals $\lambda_{i}$ with $\lambda_{1}=1.55 \mu \mathrm{m}$ and $\lambda_{2}=1.31 \mu \mathrm{m}$.

Starting from an initial shape perforated by of an array of $8 \times 8$ holes, we perform 150 iterations of our optimization algorithm, for a total computational time of approximately 20 hours. The resulting optimized design is represented on Fig. 17 (a), and the convergence history for both objective functions shown in Fig. 17 (b) - reveals that our algorithm does converge to a local optimum of the problem (6.7). The numerical simulations realized at both wavelengths $\lambda_{i}, i=1,2$ (see Fig. 17 (c,d,e)) show that the optimized design achieves approximately $85 \%$ transmission of the power contained in the incoming mode to the desired mode of the output waveguide in both considered physical settings. 


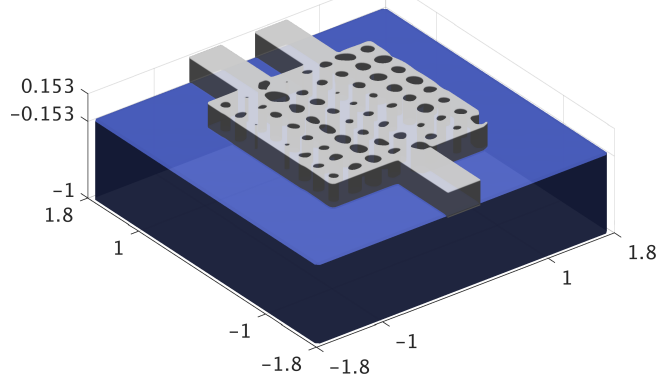

(a) Optimized shape (axes in $\mu \mathrm{m}$ )

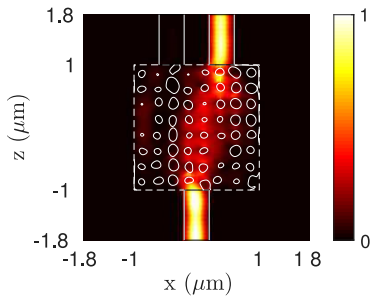

(d) Simulation at $\lambda=1.55 \mu \mathrm{m}$

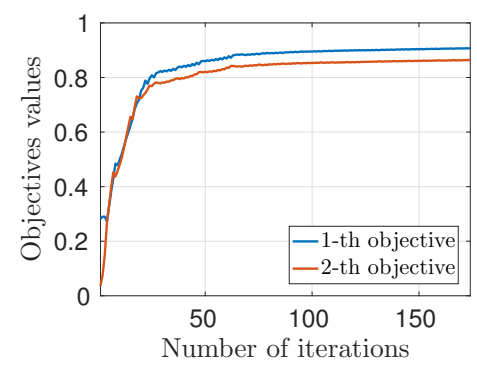

(b) Convergence history
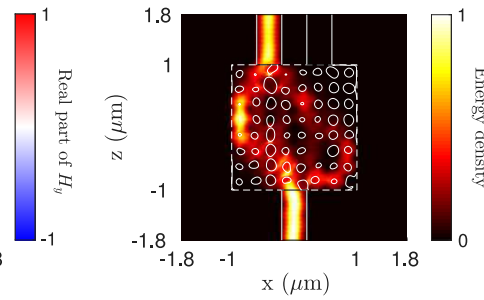

(e) Simulation at $\lambda=1.31 \mu \mathrm{m}$

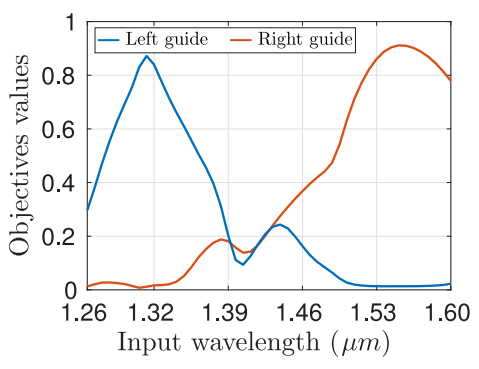

(c) Spectrum

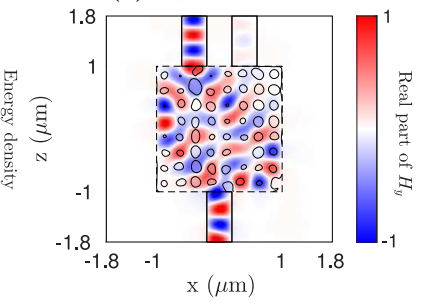

Figure 17 - Optimized design and details of the optimization process of the shape of a duplexer in Section 6.2.1.

\subsubsection{Optimization of the shape of a power divider with three output waveguides}

In this section, we consider a slightly different version of the power divider component considered in Section 6.1.3. The setting is that depicted on Fig. 18: light is injected into the device via the entrance port $\Gamma_{\text {in }}$, and the incoming power has to be equally shared between the three output ports $\Gamma_{\text {out }, i}, i=1,2,3$. Contrary to the power divider example considered in Section 6.1.3, the present optimization problem cannot be reduced to one featuring only one objective function; nevertheless, imposing shapes $\Omega \in \mathcal{U}_{\text {ad }}$ to be symmetric with respect to the $x$ axis, and restricting the considered deformations $\boldsymbol{\theta}$ in the practice of Hadamard's method to fields of the form (6.5), the problem boils down to one involving the maximization of the worst value between two objective functions:

$$
\max _{\Omega} \min _{i=1,3} J_{i}(\Omega)
$$

where $J_{i}(\Omega)$ stands for the version of $J_{o, 1}$ in which $\Gamma_{\text {obj }}$ stands for the output port $\Gamma_{\text {out }, i}$.
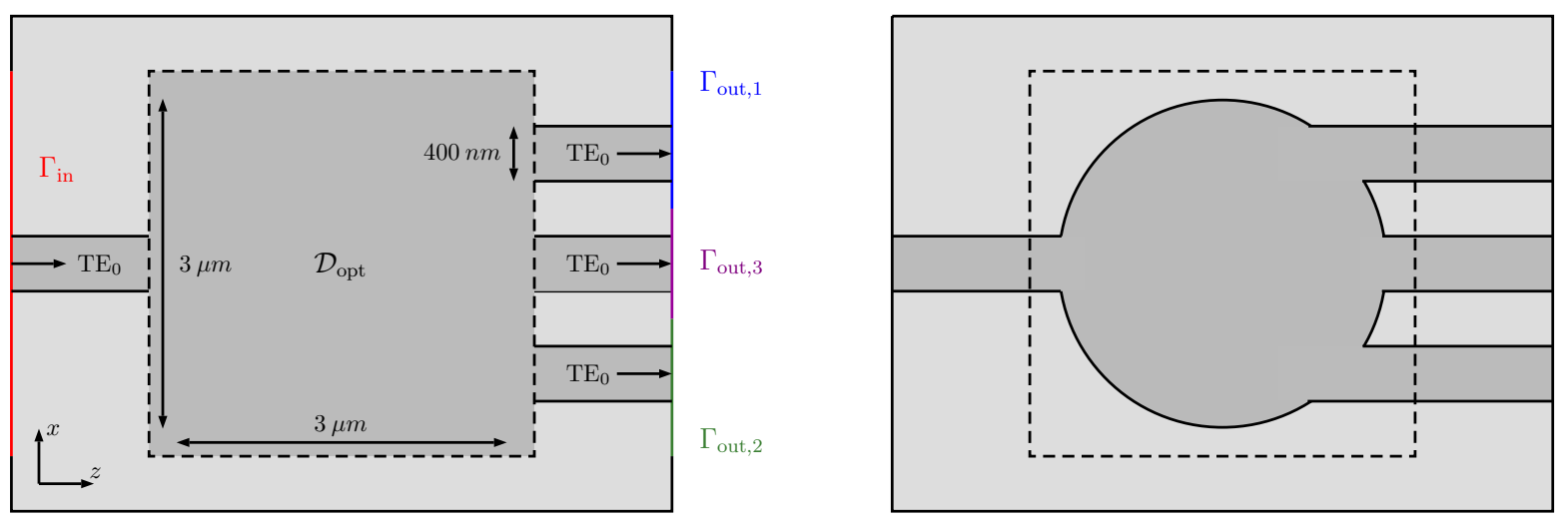

Figure 18 - Setting of the three-output power divider shape optimization problem of Section 6.2.2.

Starting from the initial shape of Fig. 18 (right), 100 iterations of our optimization algorithm are performed, for a total computational time of roughly 20 hours. The results are reported on Fig. 19. The optimized device is able to transmit approximately $30 \%$ of the incoming electromagnetic power from the entrance port $\Gamma_{\text {in }}$ into each output $\Gamma_{\text {out }, i}, i=1,2,3$. 


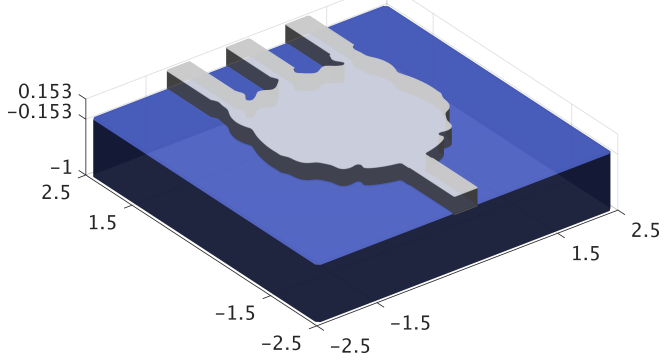

(a) Optimized shape (axes in $\mu m$ )

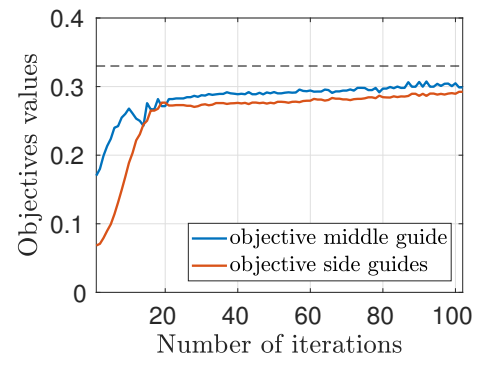

(b) Convergence history

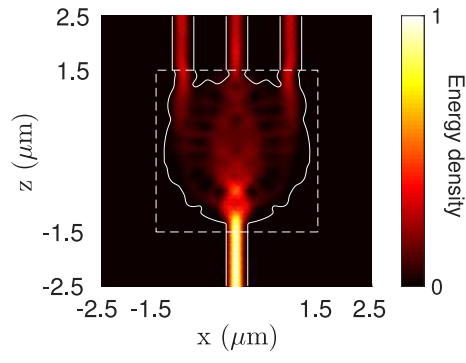

(c) Simulation at $\lambda=1.55 \mu m$

Figure 19 - Optimized design and details of the optimization process of the three-output power divider of Section 6.2.2. The dashed line in (b) represents the ideal maximum value of the performance of the device (equal to $1 / 3)$.

\subsection{Robust shape optimization of a duplexer with respect to uncertainties on the incoming wavelength}

In this section, we revisit the duplexer test-case of Section 6.2.1 with the additional concern to obtain a device whose performance is robust with respect to small perturbations, with maximum amplitude $\mathrm{m}=25 \mathrm{~nm}$, of each of the two incoming wavelengths $\lambda_{1}=1.55 \mu \mathrm{m}$ and $\lambda_{2}=1.31 \mu \mathrm{m}$.

Applying the methodology of Section 4.2, our robust optimization problem takes the form

$$
\max _{\Omega} \min _{i=1,2} \min \left\{J_{i}^{-}(\Omega), J_{i}^{+}(\Omega)\right\}
$$

where $J_{i}^{ \pm}(\Omega)$ is the function $J_{\mathrm{o}, 1}(\Omega)$ in $(3.5)$, involving the objective surface $\Gamma_{\text {obj }}=\Gamma_{\text {out }, i}$, in the situation where the operating wavelength equals $\left(\lambda_{i} \pm m\right)$.

The initial design in this example is the same as that in Section 6.2.1, i.e. it is perforated by $8 \times 8$ regularly spaced holes. After about 100 iterations of our numerical algorithm and roughly 40 hours of computation, the resulting shape is that represented on Fig. 20. Comparing the performance of this device as a function of the actual wavelength Fig. 20c to its non-robust counterpart in Fig. 17c, we observe that the new duplexer is far more robust to a small perturbation of the input wavelength.

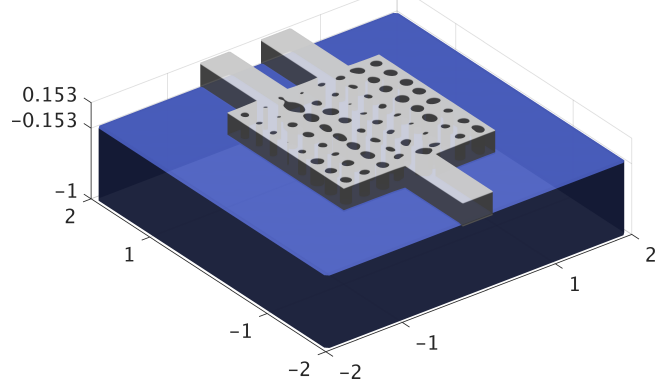

(a) Optimal shape

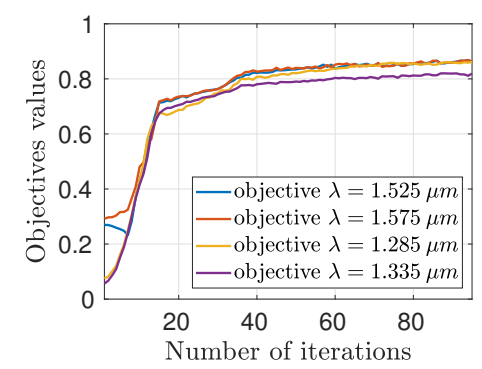

(b) Convergence history

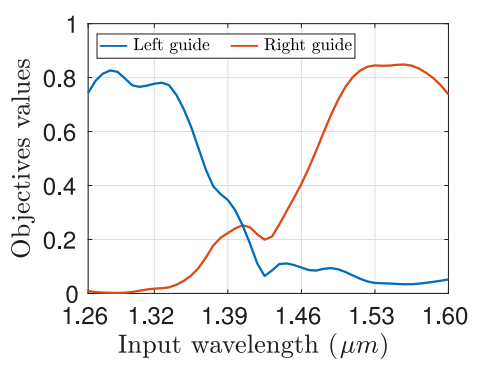

(c) Spectrum

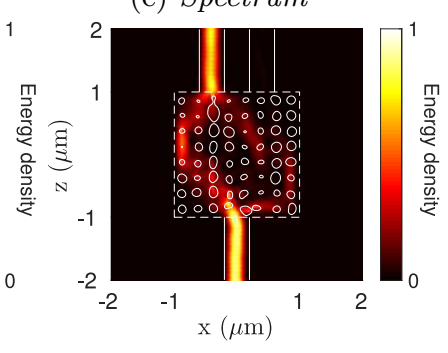

(g) Sim. at $\lambda=1.335 \mu \mathrm{m}$

(d) Sim. at $\lambda=1.525 \mu m$

(e) Sim. at $\lambda=1.575 \mu \mathrm{m}$

(f) Sim. at $\lambda=1.285 \mu m$

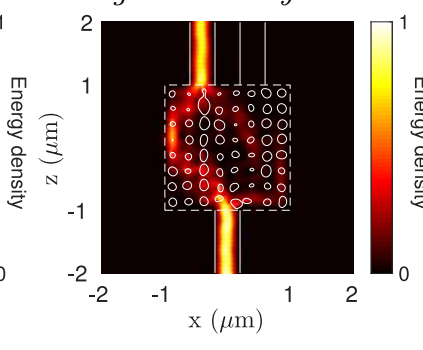

Figure 20 - Optimized shape and details of the optimization process in the duplexer test-case considering wavelength robustness of Section 6.3. 


\subsection{Robust shape optimization of nanophotonic devices with respect to geomet- ric uncertainties}

\subsubsection{Robust shape optimization of a power divider with respect to uncertainties caused by etching}

In this section we consider a variant of the power divider test-case tackled in Section 6.1.3 in which robustness of the optimized design is desired with respect to uncertainties entailed by the etching manufacturing process, as discussed in Section 4.3.1.

The shape $\Omega^{*}$ resulting from the optimization study in Section 6.1.3 (that is, without taking robustness issues into account) is not robust with respect to uncertainties related to etching. Indeed, let us consider Fig. $21 \mathrm{~b}$ where the variation of the performance criterion $J_{\mathrm{o}, 1}$ is represented when a dilation or an erosion of at most $m= \pm 30 \mathrm{~nm}$ is performed on $\Omega^{*}$ : in particular, if $\Omega^{*}$ is eroded by $30 \mathrm{~nm}$, the performance of the shape drops from $49 \%$ down to only $20 \%$.

To remedy this, starting from $\Omega^{*}$ as initial shape, we solve the following robust problem which involves the dilated and eroded versions of the optimized shape:

$$
\max _{\Omega} \min \left\{J_{-m}(\Omega), J_{0}(\Omega), J_{m}(\Omega)\right\}
$$

in which we have defined

$$
J_{\delta}(\Omega)=J_{\mathrm{o}, 1}\left(\Omega_{\delta}\right), \Omega_{\delta}:=\left(\operatorname{Id}+\delta \mathbf{n}_{\Omega}\right)(\Omega) .
$$

After 150 iterations of our optimization algorithm, we end up with the shape displayed in Fig. 21. The comparison of Fig. 21b and Fig. 21e suggests that the new power divider is much more robust to manufacturing uncertainties caused by etching. Moreover, the nominal performance of the device is not significantly degraded in the process, since it only suffers from a reduction by $1 \%$ when compared to $\Omega^{*}$. 


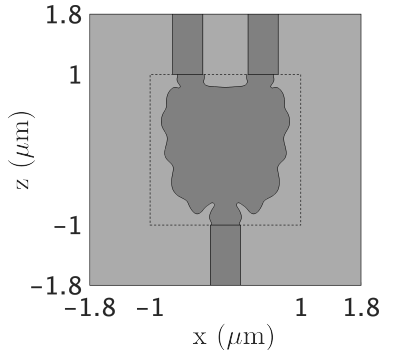

(a) Non robust optimized shape $\Omega^{*}$

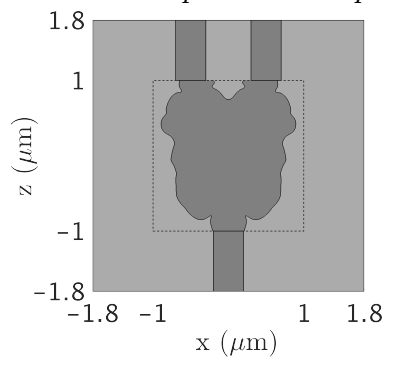

(d) Robust optimized shape

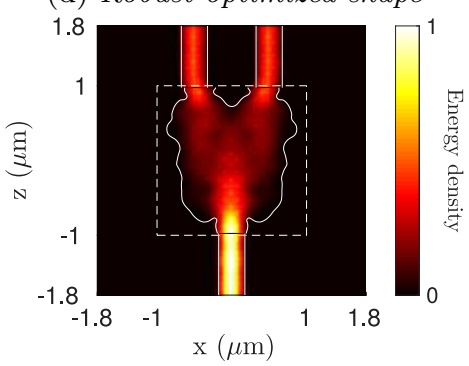

(g) Energy density eroded shape

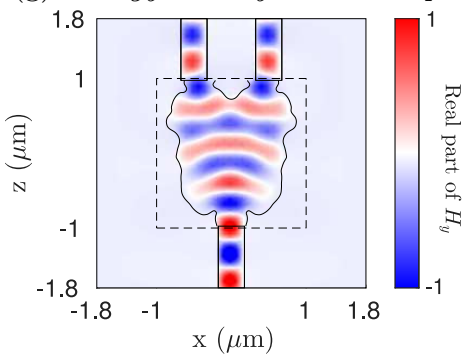

(j) Field for eroded shape

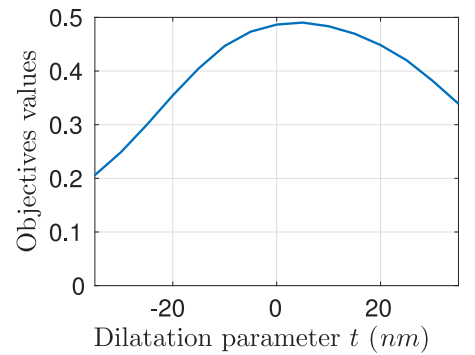

(b) Objective sensitivity to dilation

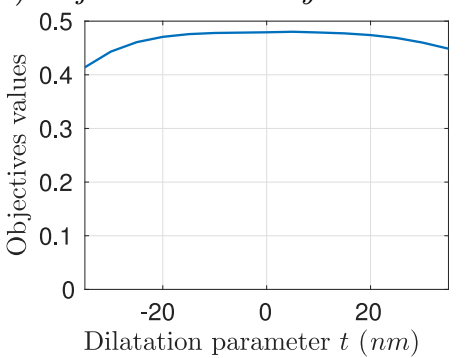

(e) Objective sensitivity to dilation

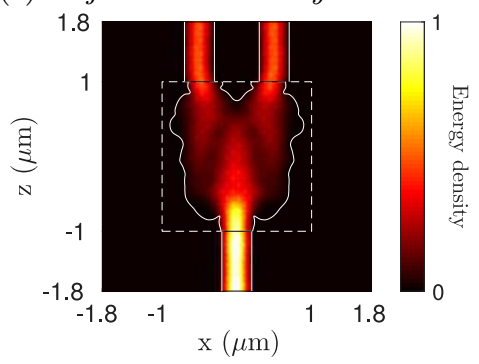

(h) Energy density optimized shape

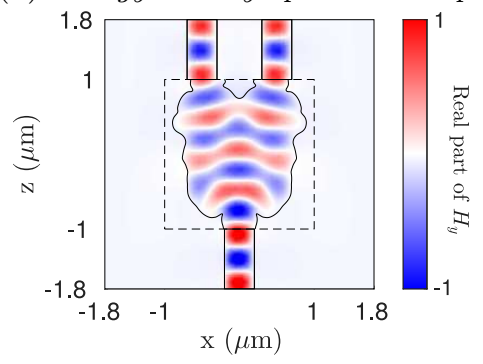

(k) Field for optimized shape

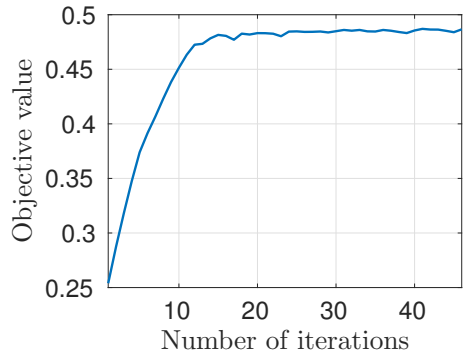

(c) Convergence graph

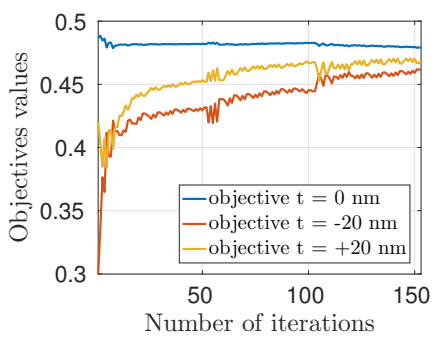

(f) Convergence history

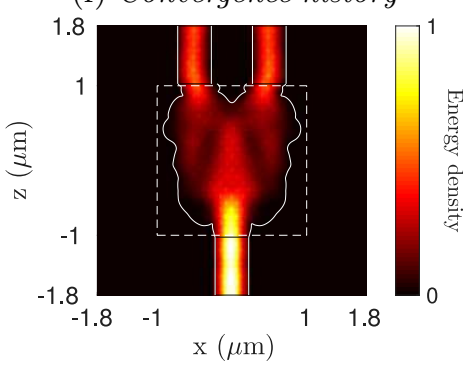

(i) Energy density dilated shape

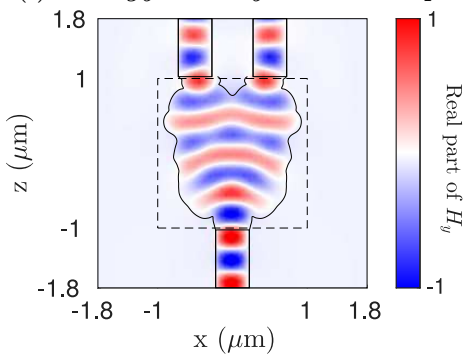

(1) Field for dilated shape

Figure 21 - Results of the robust shape optimization of a power divider with respect to uncertainties linked to etching in Section 6.4.1; the upper row reproduces the features of the non robust optimized shape $\Omega^{*}$ of Section 6.1.3.

\subsubsection{Robust optimization of a mode converter with respect to uncertainties caused by etching}

In quite the same spirit as in the previous section, we now revisit the mode converter test case of Section 6.1.2, in which the performance (6.4) of the optimized design $\Omega^{*}$ of Fig. 10 proves to be very sensitive to small perturbations of the form of a uniform dilation or erosion; see the upper row in Fig. 22.

Starting from $\Omega^{*}$ as an initial shape, we now solve the robust counterpart to the problem (6.4) involving the worst-case between the values taken by the objective function $J_{\mathrm{o}, 3}$ on the optimized shape $\Omega$ and its dilated and eroded perturbations by $20 \mathrm{~nm}$. After 200 iterations of our shape and topology optimization algorithm, the results summarized in Fig. 22 are obtained. The new optimized shape is significantly more robust to the effect of dilation and erosion; visually, its main difference with that obtained without considering robustness effects lies in that the central hole has widened to make up for the effects of dilation. 


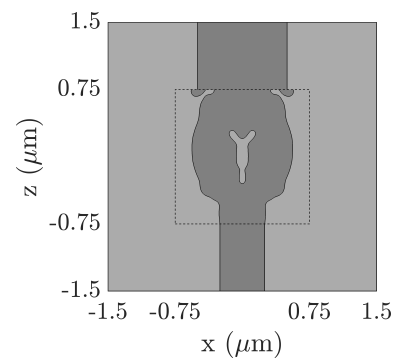

(a) Non-robust optimized shape

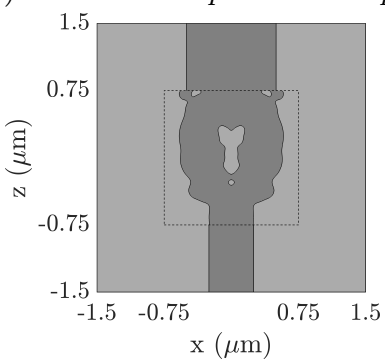

(d) Robust optimized shape

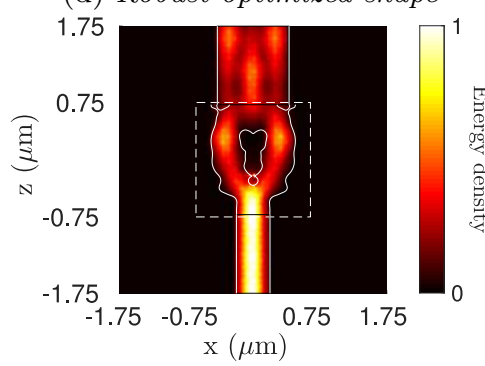

(g) Energy density eroded shape

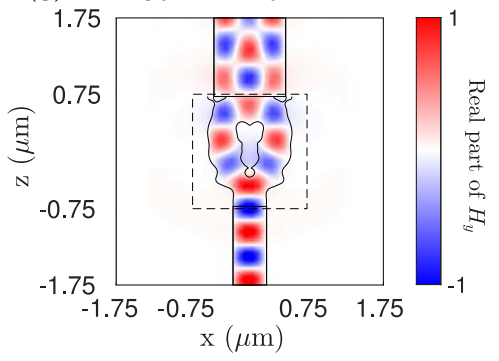

(j) Field for eroded shape

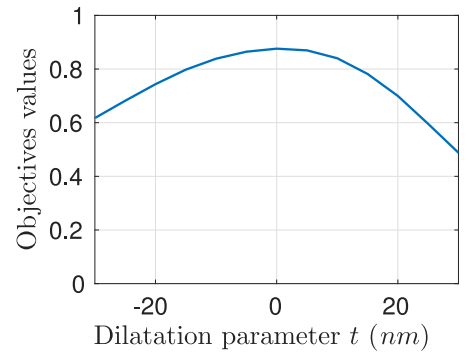

(b) Objective sensitivity to dilation

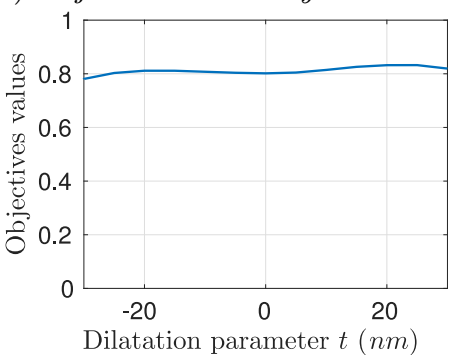

(e) Objective sensitivity to dilation

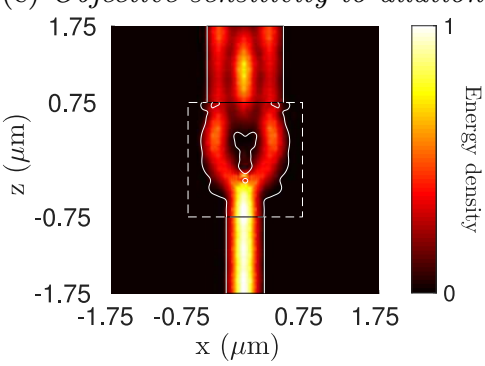

(h) Energy density optimized shape

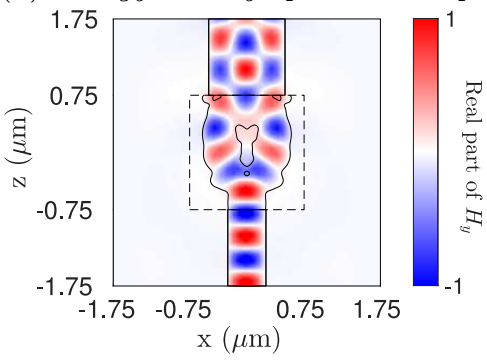

(k) Field for optimized shape

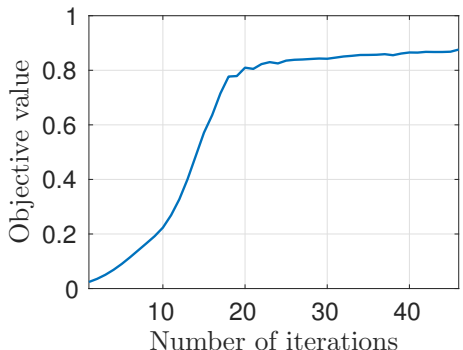

(c) Convergence history

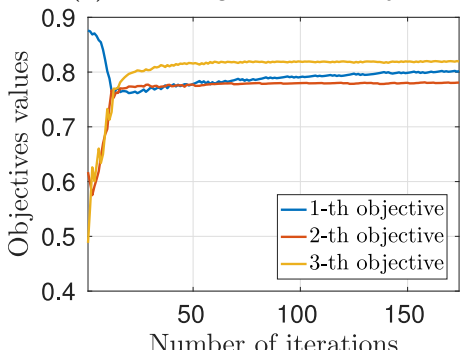

(f) Convergence history

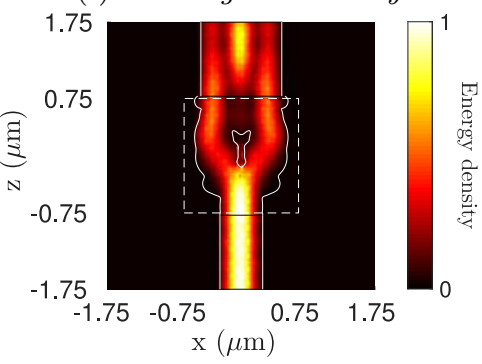

(i) Energy density dilated shape

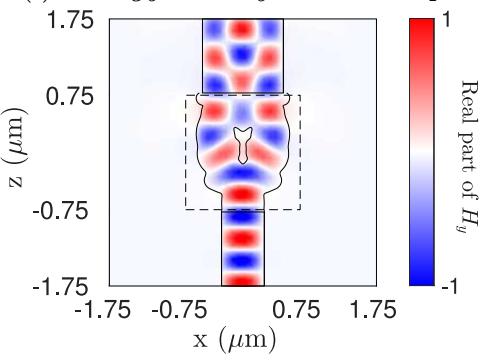

(1) Field for dilated shape

Figure 22 - Results of the robust shape optimization of a mode converter with respect to uncertainties linked to etching in Section 6.4.2; the upper row reproduces the features of the non robust optimized shape $\Omega^{*}$ of Section 6.1.2.

\subsubsection{Robust optimization of a duplexer with respect to uncertainties caused by etching}

Let us now turn again to the duplexer test case of Section 6.2.1, where taking into account robustness issues turns out to be particularly crucial. Indeed, as the results depicted in Fig. 14 and 15 have revealed, initializing the design optimization process with a shape perforated by a higher number of holes leads to an optimized shape with much better performance, as measured by the multi-objective program (6.7). Unfortunately, this effect is quite problematic from the point of view of manufacturability, since small holes like those featured by the optimized shape $\Omega^{*}$ of Fig. 17, whose radii is less than $50 \mathrm{~nm}$, are typically impossible to fabricate in practice.

So as to remedy this concern, we rely on the ideas of Section 4 , and we solve the robust counterpart to (6.7) with respect to uncertainties over a uniform dilation or erosion of shapes by a value $m=10 \mathrm{~nm}$ :

$$
\max _{\Omega} \min _{i=1,2} \min \left\{J_{i}\left(\Omega_{-m}\right), J_{i}(\Omega), J_{i}\left(\Omega_{m}\right)\right\}
$$


where we recall from Section 6.2.1 that $J_{i}(\Omega)$ is the amount of power $J_{\mathrm{o}, 1}(\Omega)$ carried by the first guided mode of the output waveguide $\Gamma_{\text {out }, i}$ when the operating wavelength is $\lambda_{i}$.

Performing 150 iterations of our shape optimization algorithm then results in the shape represented on Fig. 23, whose performance is much more robust to dilation or erosion effects than $\Omega^{*}$.

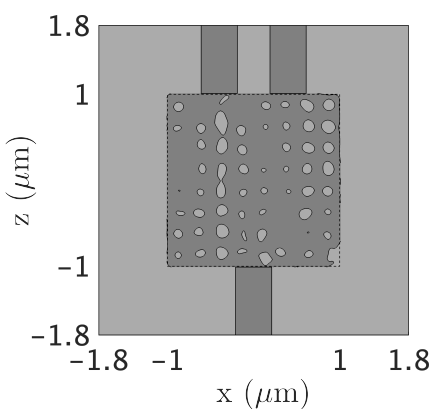

(a) Erosion by $t=-10 \mathrm{~nm}$

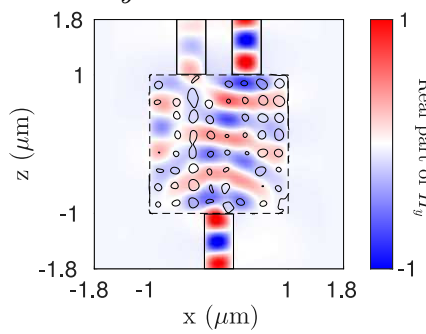

(d) $\lambda=1.55 \mu \mathrm{m}, t=-10 \mathrm{~nm}$

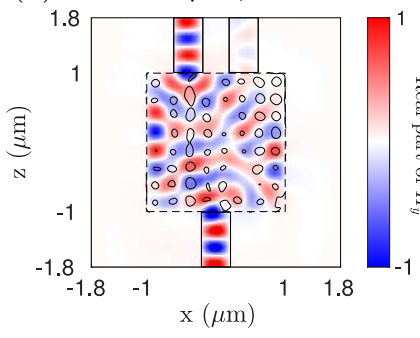

(g) $\lambda=1.31 \mu m, t=-10 \mathrm{~nm}$

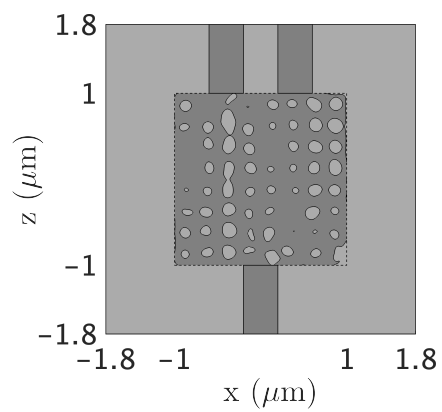

(b) Optimized shape

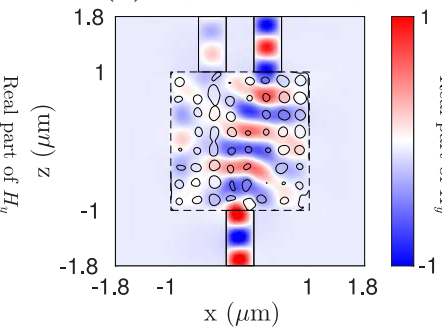

(e) $\lambda=1.55 \mu m, t=0 \mathrm{~nm}$

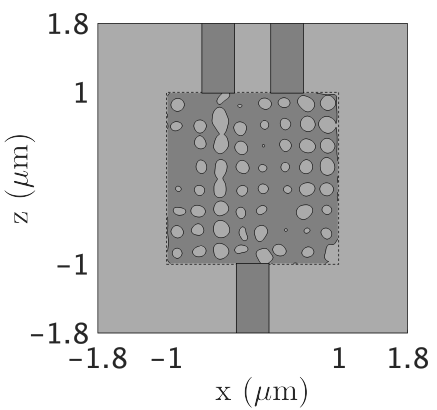

(c) Dilation by $t=+10 \mathrm{~nm}$

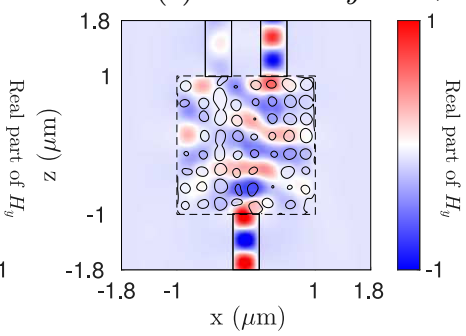

(f) $\lambda=1.55 \mu m, t=+10 \mathrm{~nm}$

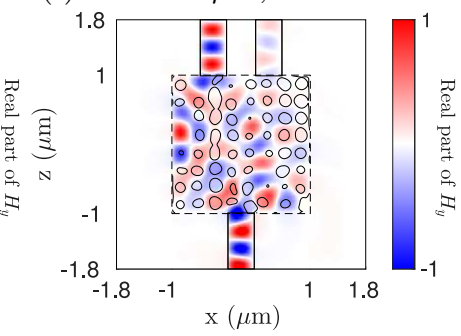

(i) $\lambda=1.31 \mu \mathrm{m}, t=+10 \mathrm{~nm}$

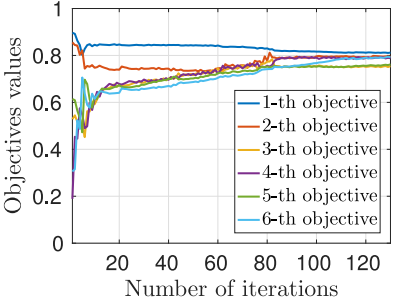

(j) Convergence history

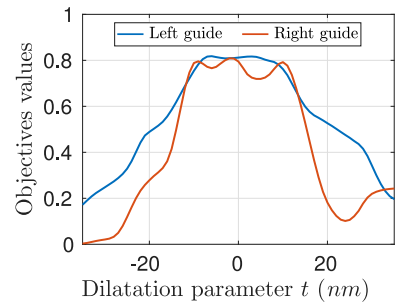

(k) Objective sensitivity to dilation / erosion

Figure 23 - Results of the robust shape optimization of a duplexer with respect to uncertainties linked to etching in Section 6.4.3.

\subsubsection{Robust optimization of a mode converter with respect to uncertainties caused by lithog- raphy}

In this last subsection we finally turn to the procedure proposed in Section 4.3.2 for imposing robustness of shapes with respect to the lithography manufacturing process. The physical setting is that of the mode converter example, as discussed in Sections 6.1.2 and 6.4.2.

We consider the robust optimization program with respect to uncertainties caused by lithography

$$
\max _{\Omega} \min \left\{J_{\mathrm{o}, 3}(\Omega), J_{\mathrm{o}, 3}\left(\widetilde{\Omega_{\delta}}\right)\right\},
$$


where we recall that the approximate perturbation $\widetilde{\Omega_{\delta}}$ of $\Omega$ is defined by (4.12), and with a value $\delta=30 \mathrm{~nm}$ for the parameter representing the standard deviation in the Gaussian kernel (4.11).

Starting from the initial shape in Fig. 9 (right) and performing 120 iterations of our optimization algorithm yields the optimized design represented on Fig. 24.

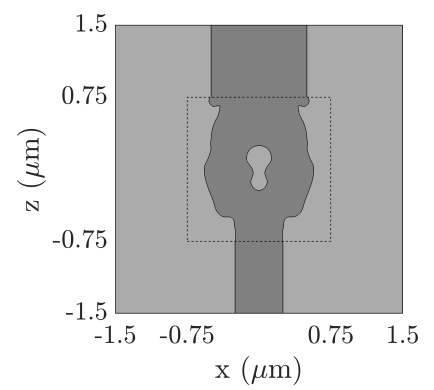

(a) Robust optimized shape with respect to lithography uncertainties

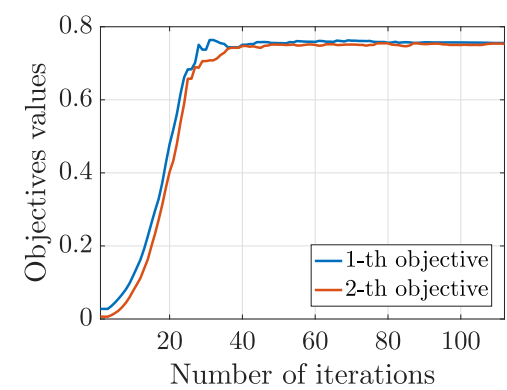

(b) Convergence history

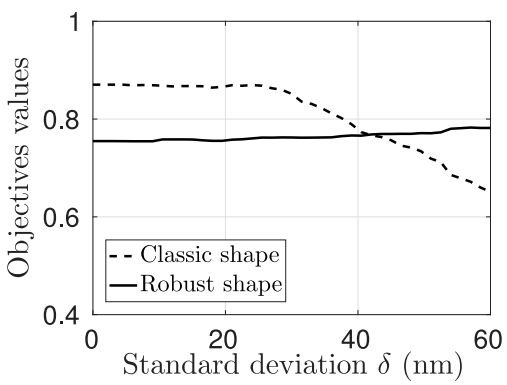

(c) Objective sensitivity to uncertainties caused by the lithography process for the non robust optimized shape in Fig. 10 and the robust one.

Figure 24 - Three dimensional lithography robust optimization of a $1.5 \times 1.5 \mu m$ mode converter.

For a simple visual comparison, the three designs obtained in this article for the mode converter test-case (non robust, robust with respect to etching uncertainties, and robust with respect to lithography uncertainties) are reproduced in Fig. 25.

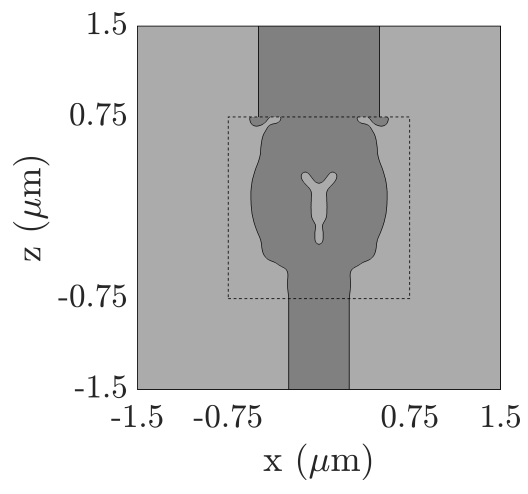

(a) Non robust (see Fig. 10)

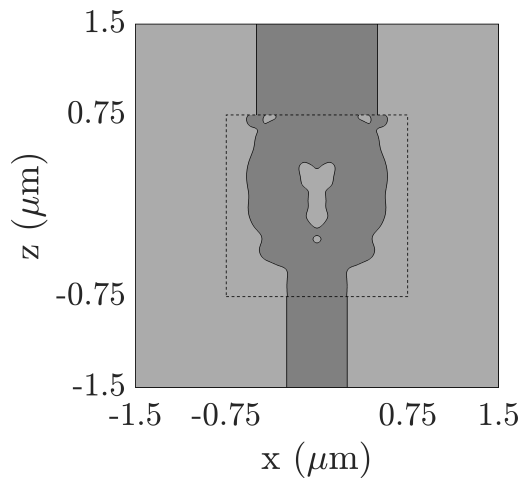

(b) Etching robust (see Fig. 22)

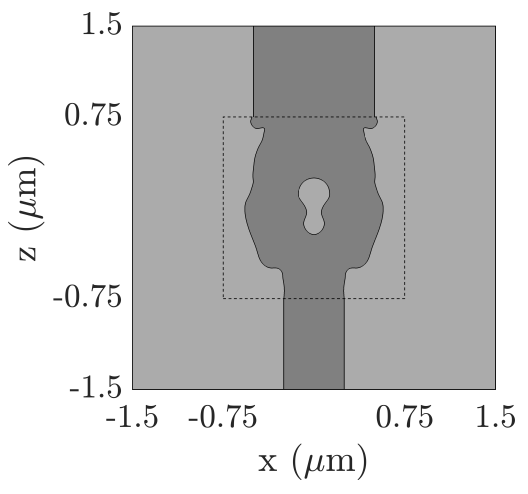

(c) Lithography robust (see Fig. 26)

Figure 25 - Optimized shapes for the mode converter example without taking robustness effects into account, and considering robustness with respect to etching and lithography uncertainties.

We also perform another optimization, using this time an initial design perforated by $8 \times 8$ holes. The results obtained in the case of the non robust optimization problem (6.4) and for its robust counterpart (6.8) are summarized in Fig. 26. As expected, for small values of $\delta$ the results are better than the one in Fig. 24 but the shapes are also more sensitive to values of $\delta$ larger than $\sim 40 \mathrm{~nm}$. 


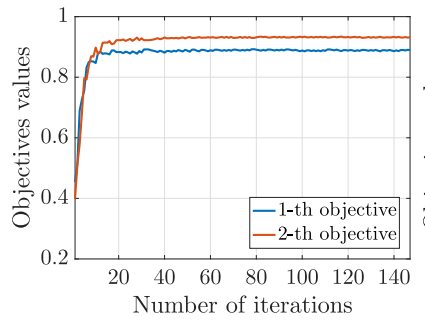

(a) Convergence history

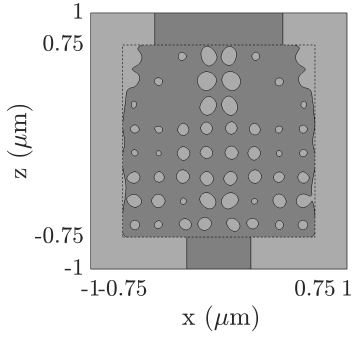

(e) Robust shape

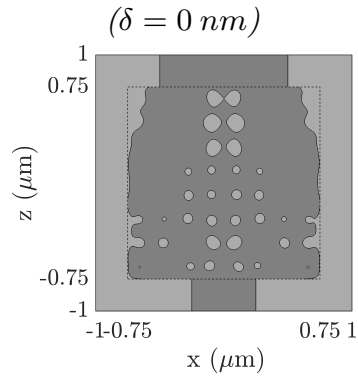

(i) Dilated robust shape

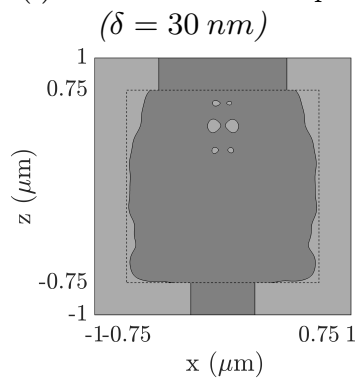

(m) Dilated robust shape $(\delta=60 \mathrm{~nm})$

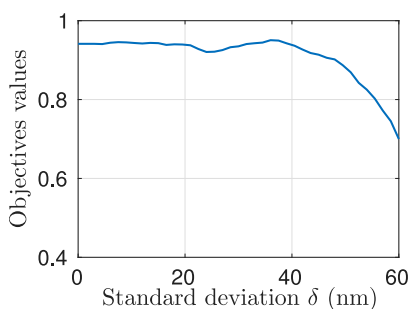

(b) Robustness to lithography

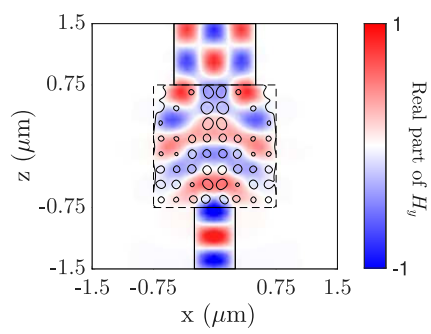

(f) Field for the robust shape $(\delta=0 \mathrm{~nm})$

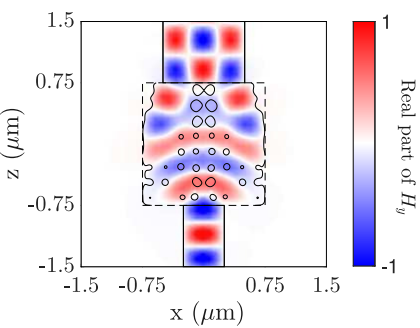

(j) Field for the dilated robust shape $(\delta=30 \mathrm{~nm})$

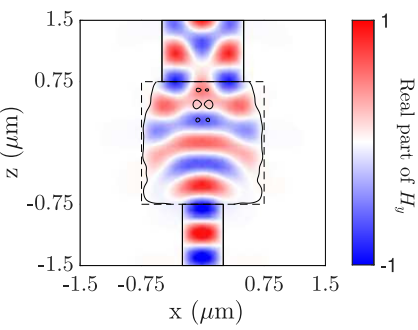

(n) Field for the dilated robust shape $(\delta=60 \mathrm{~nm})$
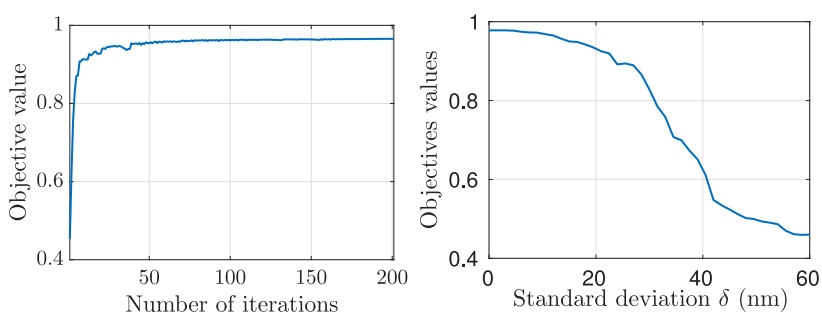

(c) Convergence history

(d) Robustness to lithography
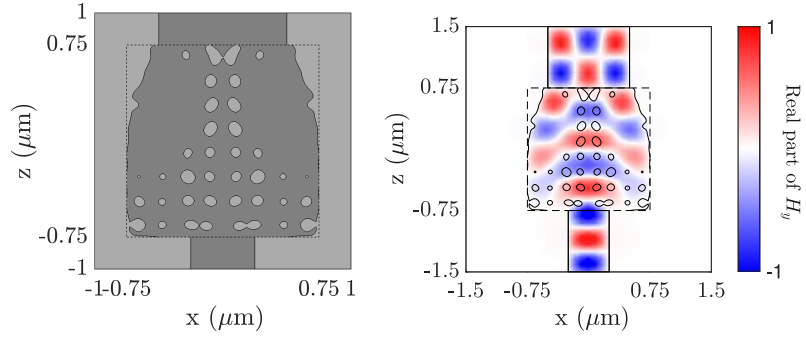

(g) Non robust shape

(h) Field for the non robust shape $(\delta=0 \mathrm{~nm})$

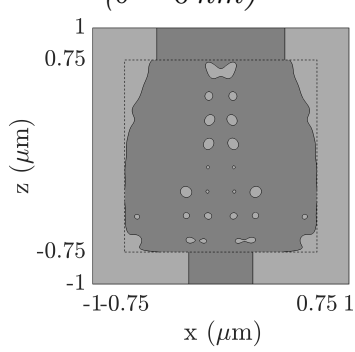

(k) Dilated non robust shape $(\delta=30 \mathrm{~nm})$

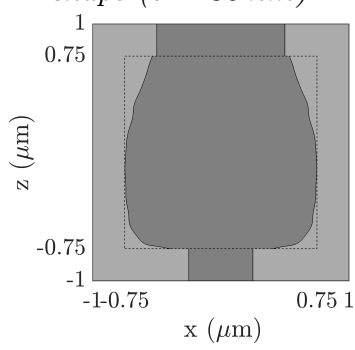

(o) Dilated non robust shape $(\delta=60 \mathrm{~nm})$

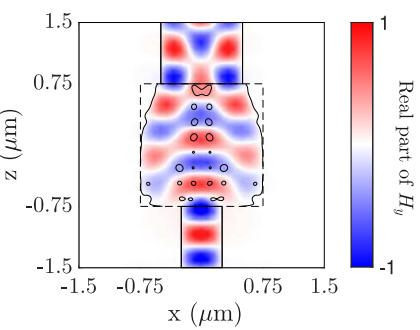

(1) Field for the dilated non robust shape $(\delta=30 \mathrm{~nm})$

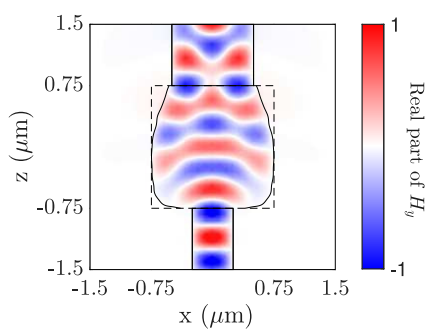

(p) Field for the dilated non robust shape $(\delta=60 \mathrm{~nm})$

Figure 26 - Optimization of the mode-converter with an initial shape of $8 \times 8$ holes, with and without lithography robustness.

\section{Conclusions and perspectives}

In this article, we have described a mathematical and numerical setting dedicated to the optimization of the shape and topology of nanophotonic components. In the context of the time-harmonic three-dimensional Maxwell equations, our framework combines Hadamard's boundary variation method when it comes to evaluating the sensitivity of a user-defined performance criterion on the shape of the arrangement of core and cladding materials within the design domain, and the level set method for tracking shapes and their deformations.

A particular attention has been paid to incorporate robustness issues in the formulation of the considered shape optimization problems. By relying on a multi-objective strategy, we have proposed a simple strategy and convenient numerical approximations to ensure that the optimized designs supplied by our algorithm have robust performances with respect to uncertainties on the incoming wavelength, or with respect 
to perturbations of their geometry in the course of the manufacturing process. The efficiency of our methods has been demonstrated on several three-dimensional examples emanating from practical applications in nanophotonics.

Several extensions of the work presented in this paper are possible. The perhaps most natural of them is about the fabrication of some of the devices optimized in Section 6, a task which has already been initiated. Another natural continuation of this work is to improve the accuracy of our numerical simulations by relying on a body-fitted mesh of shapes $\Omega$ at each stage of the optimization process. One way to achieve this goal would be to rely on the level set based mesh evolution method proposed in [5]. Eventually, from the optimization point of a view, the concurrent use of the shape derivative attached to Hadamard's method with the notion of topological derivative would make our algorithm less sensitive to the initial design.

Acknowledgements. The authors warmly thanks Dr. O. Tsilipakos and Pr. E. E. Kriezis for the fruitful discussions on the boundary conditions for mode injection. The work of the second author is partially supported by the IRS-CAOS grant from Universite Grenoble-Alpes. The third author was supported by the "Geometry and Spectral Optimization" research programme LabEx PERSYVAL-Lab GeoSpec (ANR11-LABX-0025-01) and the ANR Comedic (ANR-15-CE40-0006).

\section{Appendices}

\section{A Sketch of proof of Theorem 3.1}

Consider the functional

$$
J(\Omega)=\left|\frac{1}{4} \int_{\Gamma_{\mathrm{obj}}} j\left(\mathbf{E}_{\Omega}\right) d s\right|^{2}, \quad j\left(\mathbf{E}_{\Omega}\right):=\left(\mathbf{E}_{\Omega} \times \mathbf{H}_{m}^{*}-\frac{i}{\omega \mu_{0}} \mathbf{E}_{m}^{*} \times\left(\nabla \times \mathbf{E}_{\Omega}\right)\right) \cdot \mathbf{n},
$$

where $\mathbf{E}_{\Omega}$ belongs to the Hilbert space

$$
\mathcal{V}:=\{\mathbf{E} \in H(\operatorname{curl}, \mathcal{D}), \mathbf{n} \times \mathbf{E}=0 \text { on } \partial \mathcal{D}\},
$$

and is the unique solution in this space to the following variational problem:

$$
\forall \boldsymbol{\phi} \in \mathcal{V}, \int_{\mathcal{D}}\left(\Lambda^{-1} \nabla \times \mathbf{E}_{\Omega} \cdot \nabla \times \boldsymbol{\phi}^{*}-k^{2} n_{\Omega}^{2} \Lambda \mathbf{E}_{\Omega} \cdot \boldsymbol{\phi}^{*}\right) d x+\int_{\Gamma_{\mathrm{in}}}\left(\mathbf{U}_{\mathrm{inc}}-\gamma\left(\mathbf{E}_{\Omega}\right)\right) \cdot\left(\mathbf{n} \times \boldsymbol{\phi}^{*} \times \mathbf{n}\right) d s=0 ;
$$

see Section 2.5. In (A.2), the refractive index is given by (2.10); however, in order to simplify notations, without loss of generality judging from the way we intend to use the information contained in the shape derivative of $J(\Omega)$ (see Sections 3.1 and 3.2), we may as well assume that $n_{\Omega}$ equals the constant $n_{\text {core }}$ inside $\Omega$ and $n_{\text {clad }}$ outside $\Omega$.

The shape derivative of functionals of the form (A.1) has already been quite extensively studied in the literature (see for instance [36, 41, 61]); since the context (and so the resulting formulas) always differs slightly from one contribution to the other, we hereafter provide a rigorous sketch of proof for Theorem 3.1. In particular, one well-known difficulty in this calculation comes from the fact the electric field $\mathbf{E}_{\Omega}$ is not continuous at the interface $\partial \Omega$; the use of the formal Céa's method in this context without paying care to regularity issues is a well-known source of mistakes; see e.g. [57] about this point.

Before proceeding to the calculation of $J^{\prime}(\Omega)(\boldsymbol{\theta})$, so to speak, let us list a number of useful facts about the regularity of the electric field $\mathbf{E}_{\Omega}$ and its restrictions $\mathbf{E}_{0}$ and $\mathbf{E}_{1}$ to $\Omega$ and $\mathcal{D} \backslash \bar{\Omega}$, respectively; see for instance $[53,36]$ and $[20$, Chapter IX], about these points.

1. The restrictions $\mathbf{E}_{0}$ and $\mathbf{E}_{1}$ satisfy additional smoothness to that encoded in the spaces $H(\mathbf{c u r l}, \Omega)$ and $H(\mathbf{c u r l}, \mathcal{D} \backslash \bar{\Omega})$; this is a classical issue in the theory of elliptic partial differential equations, which follows from the smoothness of $\Omega$; typically, in our context: $\mathbf{E}_{0} \in H^{2}(\Omega)$ and $\mathbf{E}_{1} \in H^{2}(\mathcal{D})$;

2. $\mathbf{E}_{0}$ and $\mathbf{E}_{1}$ satisfy the following transmission conditions:

$$
\mathbf{n} \times \mathbf{E}_{0}=\mathbf{n} \times \mathbf{E}_{1} ; \mathbf{n} \times \nabla \times \mathbf{E}_{0}=\mathbf{n} \times \nabla \times \mathbf{E}_{1} \text {, and so } n_{\mathrm{core}}^{2} \mathbf{E}_{0} \cdot \mathbf{n}=n_{\mathrm{clad}}^{2} \mathbf{E}_{1} \cdot \mathbf{n},
$$

where the normal vector $\mathbf{n}$ to $\partial \Omega$ is oriented from $\Omega$ to $\mathcal{D} \backslash \bar{\Omega}$. 
3. The additional transmission condition holds:

$$
\nabla \times \mathbf{E}_{0} \cdot \mathbf{n}=\nabla \times \mathbf{E}_{1} \cdot \mathbf{n} \text { on } \partial \Omega ;
$$

in a nutshell, $\mathbf{E}_{0}$ and $\mathbf{E}_{1}$ are smooth inside $\Omega$ and $\mathcal{D} \backslash \bar{\Omega}$ respectively, the tangential traces $\mathbf{n} \times \nabla \times \mathbf{E}_{0}$ and $\mathbf{n} \times \nabla \times \mathbf{E}_{1}$ coincide on $\partial \Omega$ (see (A.3)) and the normal traces $\left(\nabla \times \mathbf{E}_{0}\right) \cdot \mathbf{n}$ and $\left(\nabla \times \mathbf{E}_{1}\right) \cdot \mathbf{n}$ are easily seen to be continuous across $\partial \Omega$ (they involve only tangential derivatives of the tangential components of $\mathbf{E}_{\Omega}$ and $\mathbf{A}_{\Omega}$ on $\partial \Omega$ ). As a result, the quantity $\nabla \times \mathbf{E}_{\Omega}$ belongs to $H^{1}(\mathcal{D})$.

Let us eventually mention that the exact counterparts to these relations hold for the adjoint state $\mathbf{A}_{\Omega}$.

In order to prove the shape differentiability of $J(\Omega)$, we first calculate the "Lagrangian" derivative of the mapping $\Omega \mapsto \mathbf{E}_{\Omega}$; namely, for $\boldsymbol{\theta} \in \Theta_{\text {ad }}$, let us introduce the transported field

$$
\overline{\mathbf{E}_{\boldsymbol{\theta}}}:=\left(I+\nabla \boldsymbol{\theta}^{T}\right) \mathbf{E}_{\Omega_{\boldsymbol{\theta}}} \circ(\mathrm{Id}+\boldsymbol{\theta}) .
$$

Then, $\mathbf{E}_{\Omega_{\boldsymbol{\theta}}}$ belongs to $\mathcal{V}$ if and only if $\overline{\mathbf{E}_{\boldsymbol{\theta}}}$ belongs to $\mathcal{V}$ and:

$$
\left(\nabla \times \mathbf{E}_{\Omega_{\boldsymbol{\theta}}}\right) \circ(\mathrm{Id}+\boldsymbol{\theta})=\frac{1}{|\operatorname{det}(\mathrm{I}+\nabla \boldsymbol{\theta})|}(\mathrm{I}+\nabla \boldsymbol{\theta})\left(\nabla \times \overline{\mathbf{E}}_{\boldsymbol{\theta}}\right) ;
$$

see for instance [53, Corollary 3.58]. Let us now perform a change of variables in the variational formulation (A.2) for $\mathbf{E}_{\Omega_{\theta}}$; taking into account (A.4), the relation

$$
n_{\Omega}=n_{\Omega_{\boldsymbol{\theta}}} \circ(\mathrm{Id}+\boldsymbol{\theta}),
$$

and the fact that $\boldsymbol{\theta} \equiv 0$ on $\mathcal{D}_{\mathrm{PML}}$ and $\Gamma_{\text {in }}$, we obtain:

$$
\begin{aligned}
\forall \boldsymbol{\phi} \in \mathcal{V}, \int_{\mathcal{D}}\left(\Lambda^{-1}\left(A(\boldsymbol{\theta}) \nabla \times \overline{\mathbf{E}_{\boldsymbol{\theta}}}\right) \cdot \nabla \times \overline{\boldsymbol{\phi}}_{\theta}^{*}\right) d x-\int_{\mathcal{D}} k^{2} C(\boldsymbol{\theta}) \Lambda n_{\Omega}^{2} \overline{\mathbf{E}_{\boldsymbol{\theta}}} \cdot \overline{\boldsymbol{\phi}}_{\boldsymbol{\theta}}^{*} d x \\
\quad+\int_{\Gamma_{\mathrm{in}}}\left(\mathbf{U}_{\mathrm{inc}}-\gamma\left(\overline{\mathbf{E}_{\boldsymbol{\theta}}}\right)\right) \cdot \mathbf{n} \times \overline{\boldsymbol{\phi}}_{\boldsymbol{\theta}}^{*} \times \mathbf{n} d s=0,
\end{aligned}
$$

in which we have used the shortcut

$$
\overline{\boldsymbol{\phi}}_{\boldsymbol{\theta}}:=\left(I+\nabla \boldsymbol{\theta}^{T}\right) \boldsymbol{\phi} \circ(\mathrm{Id}+\boldsymbol{\theta}),
$$

and where the matrices $A(\boldsymbol{\theta})$ and $C(\boldsymbol{\theta})$ are defined by:

$$
A(\boldsymbol{\theta})=C(\boldsymbol{\theta})^{-1}=\frac{1}{|\operatorname{det}(\mathrm{I}+\nabla \boldsymbol{\theta})|}\left(\mathrm{I}+\nabla \boldsymbol{\theta}^{T}\right)(\mathrm{I}+\nabla \boldsymbol{\theta}) .
$$

Note that since $\phi \in \mathcal{V}$ is arbitrary in (A.2), then so is $\bar{\phi}_{\theta} \in \mathcal{V}$, and so:

$$
\begin{aligned}
\forall \boldsymbol{\phi} \in \mathcal{V}, \int_{\mathcal{D}}\left(\Lambda^{-1}\left(A(\boldsymbol{\theta}) \nabla \times \overline{\mathbf{E}_{\boldsymbol{\theta}}}\right) \cdot \nabla \times \boldsymbol{\phi}^{*}\right) d x-\int_{\mathcal{D}} k^{2} C(\boldsymbol{\theta}) \Lambda n_{\Omega}^{2} \overline{\mathbf{E}_{\boldsymbol{\theta}}} \cdot \boldsymbol{\phi}^{*} d x \\
\quad+\int_{\Gamma_{\mathrm{in}}}\left(\mathbf{U}_{\mathrm{inc}}-\gamma\left(\overline{\mathbf{E}_{\boldsymbol{\theta}}}\right)\right) \cdot \mathbf{n} \times \boldsymbol{\phi}^{*} \times \mathbf{n} d s=0,
\end{aligned}
$$

Now, a classical use of the implicit function theorem, as in e.g. [35, 54], reveals that the mapping $\boldsymbol{\theta} \mapsto \overline{\mathbf{E}}_{\boldsymbol{\theta}}$ is Fréchet differentiable from $\Theta_{\text {ad }}$ into $\mathcal{V}$. Its derivative $\stackrel{\circ}{\mathbf{E}}_{\Omega}(\boldsymbol{\theta}) \in \mathcal{V}$ - the Lagrangian derivative of $\Omega \mapsto \mathbf{E}_{\Omega}$ - is the unique solution to the following variational problem: for all $\phi \in \mathcal{V}$,

$$
\begin{array}{r}
\int_{\mathcal{D}}\left(\Lambda^{-1}\left(\nabla \times \stackrel{\circ}{\mathbf{E}}_{\Omega}(\boldsymbol{\theta})\right) \cdot \nabla \times \boldsymbol{\phi}^{*}\right) d x-\int_{\mathcal{D}} k^{2} \Lambda n_{\Omega}^{2} \stackrel{\circ}{\mathbf{E}}_{\Omega}(\boldsymbol{\theta}) \cdot \boldsymbol{\phi}^{*} d x-\int_{\Gamma_{\mathrm{in}}} \gamma\left(\mathbf{E}_{\Omega}(\boldsymbol{\theta})\right) \cdot \mathbf{n} \times \boldsymbol{\phi}^{*} \times \mathbf{n} d s= \\
\int_{\mathcal{D}}\left(\Lambda^{-1}\left(C^{\prime}(0)(\boldsymbol{\theta}) \nabla \times \mathbf{E}_{\Omega}\right) \cdot \nabla \times \boldsymbol{\phi}^{*}\right) d x+\int_{\mathcal{D}} k^{2} \Lambda C^{\prime}(0)(\boldsymbol{\theta}) n_{\Omega}^{2} \mathbf{E}_{\Omega} \cdot \boldsymbol{\phi}^{*} d x .
\end{array}
$$

In the calculation of (A.6), we have used the derivative

$$
A^{\prime}(0)(\boldsymbol{\theta})=-C^{\prime}(0)(\boldsymbol{\theta})=-(\operatorname{div} \boldsymbol{\theta}) \mathrm{I}+\nabla \boldsymbol{\theta}+\nabla \boldsymbol{\theta}^{T} .
$$


Let us now use this information to calculate the shape derivative of $J(\Omega)$. A change of variables combined with the fact that $\boldsymbol{\theta} \equiv 0$ on $\Gamma_{\text {obj }}$ yields:

$$
J\left(\Omega_{\boldsymbol{\theta}}\right)=\frac{1}{16}\left(\int_{\Gamma_{\mathrm{obj}}} j\left(\overline{\mathbf{E}_{\theta}}\right) d s\right)\left(\int_{\Gamma_{\mathrm{obj}}} j\left(\overline{\mathbf{E}_{\theta}}\right) d s\right)^{*} .
$$

Differentiating with respect to $\boldsymbol{\theta}$ in the above expression results in:

$$
J^{\prime}(\Omega)(\boldsymbol{\theta})=\frac{1}{8} \mathcal{R} e\left[\left(\int_{\Gamma_{\mathrm{obj}}} j\left(\stackrel{\circ}{\mathbf{E}}_{\Omega}(\boldsymbol{\theta})\right) d s\right)\left(\int_{\Gamma_{\mathrm{obj}}} j\left(\mathbf{E}_{\Omega}\right) d s\right)^{*}\right],
$$

where we recall that the mapping $\mathbf{E} \mapsto j(\mathbf{E})$ is linear. Now let us introduce the adjoint state $\mathbf{A}_{\Omega}$, unique solution in $\mathcal{V}$ to the following system

$$
\begin{aligned}
\int_{\mathcal{D}}\left(\Lambda^{-1}\left(\nabla \times \mathbf{A}_{\Omega}\right) \cdot \nabla \times \phi^{*}\right) d x-\int_{\mathcal{D}} k^{2} \Lambda n_{\Omega}^{2} \mathbf{A}_{\Omega} \cdot \phi^{*} d x-\int_{\Gamma_{\mathrm{in}}} \gamma\left(\mathbf{A}_{\Omega}\right) \cdot \mathbf{n} \times \boldsymbol{\phi}^{*} \times \mathbf{n} d s= \\
-\frac{1}{8}\left(\int_{\Gamma_{\mathrm{obj}}} j(\boldsymbol{\phi}) d s\right)\left(\int_{\Gamma_{\mathrm{obj}}} j\left(\mathbf{E}_{\Omega}\right) d s\right)^{*} .
\end{aligned}
$$

Then using (A.6) with $\boldsymbol{\phi}=\boldsymbol{A}_{\Omega}$ and (A.8) with $\boldsymbol{\phi}=\stackrel{\circ}{\mathbf{E}}_{\Omega}(\boldsymbol{\theta})$, the expression (A.7) of the shape derivative of $J(\Omega)$ rewrites:

$$
J^{\prime}(\Omega)(\boldsymbol{\theta})=-\mathcal{R} e\left[\int_{\mathcal{D}}\left(\Lambda^{-1}\left(C^{\prime}(0)(\boldsymbol{\theta}) \nabla \times \mathbf{E}_{\Omega}\right) \cdot \nabla \times \mathbf{A}_{\Omega}^{*}\right) d x+\int_{\mathcal{D}} k^{2} \Lambda C^{\prime}(0)(\boldsymbol{\theta}) n_{\Omega}^{2} \mathbf{E}_{\Omega} \cdot \mathbf{A}_{\Omega}^{*} d x\right] .
$$

Formula (A.9) is the so-called volumetric expression of the shape derivative $J^{\prime}(\Omega)(\boldsymbol{\theta})$. We now simplify this expression to achieve a more convenient surfacic expression, obeying the structure theorem. To this end, we follow the strategy of [28]: we perform integration by parts to obtain an expression of $J^{\prime}(\Omega)(\boldsymbol{\theta})$ of the form

$$
J^{\prime}(\Omega)(\boldsymbol{\theta})=\int_{\partial \Omega} g\left(\mathbf{E}_{\Omega}, \mathbf{A}_{\Omega}\right) \boldsymbol{\theta} \cdot \mathbf{n} d s+\int_{\mathcal{D}} r\left(\mathbf{E}_{\Omega}, \mathbf{A}_{\Omega}\right) \cdot \boldsymbol{\theta} d x,
$$

for some functions $g\left(\mathbf{E}_{\Omega}, \mathbf{A}_{\Omega}\right) \in L^{1}(\partial \Omega)$ and $r\left(\mathbf{E}_{\Omega}, \mathbf{A}_{\Omega}\right) \in L^{1}(\mathcal{D})$ depending on $\mathbf{E}_{\Omega}$ and $\mathbf{A}_{\Omega}$. Relying on the structure theorem, we then identify that the second integral in the above right-hand side must vanish. In the following, we denote by $r\left(\mathbf{E}_{\Omega}, \mathbf{A}_{\Omega}\right)$ any function in $L^{1}(\mathcal{D})$ (possibly changing from one line to the other) which depends on $\mathbf{E}_{\Omega}$ and $\mathbf{A}_{\Omega}$.

We rely on the following integration by parts formulas, valid for smooth enough vector fields $\boldsymbol{\theta}, \mathbf{u}, \mathbf{v}$ :

$$
\begin{gathered}
\int_{\Omega}(\operatorname{div} \boldsymbol{\theta}) \mathbf{u} \cdot \mathbf{v} d x=\int_{\partial \Omega} \mathbf{u} \cdot \mathbf{v}(\boldsymbol{\theta} \cdot \mathbf{n}) d s-\int_{\Omega} \nabla(\mathbf{u} \cdot \mathbf{v}) \cdot \boldsymbol{\theta} d x \\
\int_{\Omega}(\nabla \boldsymbol{\theta} \mathbf{u}) \cdot \mathbf{v} d x=\int_{\partial \Omega}(\boldsymbol{\theta} \cdot \mathbf{v})(\mathbf{u} \cdot \mathbf{n}) d s-\int_{\Omega}(\operatorname{div} \mathbf{u}) \boldsymbol{\theta} \cdot \mathbf{v} d x-\int_{\Omega}(\nabla \mathbf{v u}) \cdot \boldsymbol{\theta} d x \\
\int_{\Omega}\left(\nabla \boldsymbol{\theta}^{T} \mathbf{u}\right) \cdot \mathbf{v} d x=\int_{\partial \Omega}(\boldsymbol{\theta} \cdot \mathbf{u})(\mathbf{v} \cdot \mathbf{n}) d s-\int_{\Omega}(\operatorname{div} \mathbf{v}) \boldsymbol{\theta} \cdot \mathbf{u} d x-\int_{\Omega}(\nabla \mathbf{u v}) \cdot \boldsymbol{\theta} d x
\end{gathered}
$$

At first, using the fact that $\nabla \times \mathbf{E}_{\Omega}$ and $\nabla \times \mathbf{A}_{\Omega}$ belongs to $H^{1}(\mathcal{D})$, an integration by parts based on (A.10) to (A.12) and the fact that $\boldsymbol{\theta} \equiv 0$ on $\partial \mathcal{D}$ yields:

$$
\int_{\mathcal{D}}\left(\Lambda^{-1}\left(C^{\prime}(0)(\boldsymbol{\theta}) \nabla \times \mathbf{E}_{\Omega}\right) \cdot \nabla \times \mathbf{A}_{\Omega}^{*}\right) d x=\int_{\mathcal{D}} \boldsymbol{\theta} \cdot r\left(\mathbf{E}_{\Omega}, \mathbf{A}_{\Omega}\right) d x
$$

for some $r\left(\mathbf{E}_{\Omega}, \mathbf{A}_{\Omega}\right) \in L^{1}(\mathcal{D})$. Then,

$$
\begin{aligned}
& \int_{\mathcal{D}} k^{2} \Lambda C^{\prime}(0)(\boldsymbol{\theta}) n_{\Omega}^{2} \mathbf{E}_{\Omega} \cdot \mathbf{A}_{\Omega}^{*} d x= \\
& \quad \int_{\Omega} k^{2}\left(\operatorname{div} \boldsymbol{\theta} \mathrm{I}-\nabla \boldsymbol{\theta}-\nabla \boldsymbol{\theta}^{T}\right) n_{\mathrm{core}}^{2} \mathbf{E}_{0} \cdot \mathbf{A}_{0}^{*} d x+\int_{\mathcal{D} \backslash \bar{\Omega}} k^{2} \Lambda\left(\operatorname{div} \boldsymbol{\theta} \mathrm{I}-\nabla \boldsymbol{\theta}-\nabla \boldsymbol{\theta}^{T}\right) n_{\mathrm{clad}}^{2} \mathbf{E}_{1} \cdot \mathbf{A}_{1}^{*} d x
\end{aligned}
$$


whence since $\mathbf{E}_{0}, \mathbf{A}_{0}$ (resp. $\mathbf{E}_{1}, \mathbf{A}_{1}$ ) are smooth on $\Omega$ (resp. $\mathcal{D} \backslash \bar{\Omega}$ ) and an integration by parts yields

$$
\begin{aligned}
\int_{\Omega} & k^{2} n_{\text {core }}^{2}(\operatorname{div} \boldsymbol{\theta}) \mathbf{E}_{0} \cdot \mathbf{A}_{0}^{*} d x+\int_{\mathcal{D} \backslash \bar{\Omega}} k^{2} n_{\text {clad }}^{2} \Lambda(\operatorname{div} \boldsymbol{\theta}) \mathbf{E}_{1} \cdot \mathbf{A}_{1}^{*} d x \\
= & \int_{\partial \Omega} k^{2}\left(n_{\text {core }}^{2} \mathbf{E}_{0} \cdot \mathbf{A}_{0}^{*}-n_{\text {clad }}^{2} \mathbf{E}_{1} \cdot \mathbf{A}_{1}^{*}\right)(\boldsymbol{\theta} \cdot \mathbf{n}) d s+\int_{\partial \Omega} \boldsymbol{\theta} \cdot r\left(\mathbf{E}_{\Omega}, \mathbf{A}_{\Omega}\right) d x \\
= & \int_{\partial \Omega}\left(k^{2}\left(n_{\text {core }}^{2}-n_{\text {clad }}^{2}\right)\left(\mathbf{n} \times \mathbf{E}_{\Omega} \times \mathbf{n}\right) \cdot\left(\mathbf{n} \times \mathbf{A}_{\Omega}^{*} \times \mathbf{n}\right)+k^{2}\left(\frac{1}{n_{\text {core }}^{2}}-\frac{1}{n_{\text {clad }}^{2}}\right)\left(n_{\Omega}^{2} \mathbf{E}_{\Omega} \cdot \mathbf{n}\right)\left(n_{\Omega}^{2} \mathbf{A}_{\Omega}^{*} \cdot n\right)\right)(\boldsymbol{\theta} \cdot \mathbf{n}) d s \\
& +\int_{\mathcal{D}} \boldsymbol{\theta} \cdot r\left(\mathbf{E}_{\Omega}, \mathbf{A}_{\Omega}\right) d x
\end{aligned}
$$

where we have used the continuity (A.3) of the tangential components of $\mathbf{E}_{\Omega}$ and $\mathbf{A}_{\Omega}$, and that of the normal component $n_{\Omega}^{2} \mathbf{E}_{\Omega} \cdot \mathbf{n}$ of the electric displacement.

In a similar fashion, we calculate:

$$
\begin{aligned}
& \int_{\Omega} k^{2} n_{\text {core }}^{2}\left(\nabla \boldsymbol{\theta} \mathbf{E}_{0}\right) \cdot \mathbf{A}_{0}^{*} d x+\int_{\mathcal{D} \backslash \bar{\Omega}} k^{2} n_{\text {clad }}^{2} \Lambda\left(\nabla \boldsymbol{\theta} \mathbf{E}_{1}\right) \cdot \mathbf{A}_{1}^{*} d x= \\
& \int_{\partial \Omega} k^{2}\left(n_{\text {core }}^{2}\left(\mathbf{E}_{0} \cdot \mathbf{n}\right)\left(\mathbf{A}_{0}^{*} \cdot \boldsymbol{\theta}\right)-n_{\text {clad }}^{2}\left(\mathbf{E}_{1} \cdot \mathbf{n}\right)\left(\mathbf{A}_{1}^{*} \cdot \boldsymbol{\theta}\right)\right) d s+\int_{\mathcal{D}} \boldsymbol{\theta} \cdot r\left(\mathbf{E}_{\Omega}, \mathbf{A}_{\Omega}\right) d x .
\end{aligned}
$$

Using (A.3), this rewrites:

$$
\begin{aligned}
\int_{\Omega} k^{2} n_{\text {core }}^{2}\left(\nabla \boldsymbol{\theta} \mathbf{E}_{0}\right) \cdot \mathbf{A}_{0}^{*} d x+\int_{\mathcal{D} \backslash \bar{\Omega}} k^{2} n_{\text {clad }}^{2}\left(\nabla \boldsymbol{\theta} \mathbf{E}_{1}\right) \cdot \mathbf{A}_{1}^{*} d x= \\
\int_{\partial \Omega} k^{2}\left(\frac{1}{n_{\text {core }}^{2}}-\frac{1}{n_{\text {clad }}^{2}}\right)\left(n_{\Omega}^{2} \mathbf{E}_{\Omega} \cdot \mathbf{n}\right)\left(n_{\Omega}^{2} \mathbf{A}_{\Omega}^{*} \cdot \mathbf{n}\right)(\boldsymbol{\theta} \cdot \mathbf{n}) d s+\int_{\mathcal{D}} \boldsymbol{\theta} \cdot r\left(\mathbf{E}_{\Omega}, \mathbf{A}_{\Omega}\right) d x .
\end{aligned}
$$

In the same way, we obtain:

$$
\begin{aligned}
\int_{\Omega} k^{2} n_{\text {core }}^{2}\left(\nabla \boldsymbol{\theta}^{T} \mathbf{E}_{0}\right) \cdot \mathbf{A}_{0}^{*} d x+\int_{\mathcal{D} \backslash \bar{\Omega}} k^{2} n_{\text {clad }}^{2}\left(\nabla \boldsymbol{\theta}^{T} \mathbf{E}_{1}\right) \cdot \mathbf{A}_{1}^{*} d x= \\
\int_{\partial \Omega} k^{2}\left(\frac{1}{n_{\text {core }}^{2}}-\frac{1}{n_{\text {clad }}^{2}}\right)\left(n_{\Omega}^{2} \mathbf{E}_{\Omega} \cdot \mathbf{n}\right)\left(n_{\Omega}^{2} \mathbf{A}_{\Omega}^{*} \cdot \mathbf{n}\right)(\boldsymbol{\theta} \cdot \mathbf{n}) d s+\int_{\mathcal{D}} \boldsymbol{\theta} \cdot r\left(\mathbf{E}_{\Omega}, \mathbf{A}_{\Omega}\right) d x .
\end{aligned}
$$

Combining (A.13) to (A.16), we obtain:

$$
\begin{aligned}
& \int_{\mathcal{D}} k^{2} \Lambda C^{\prime}(0)(\boldsymbol{\theta}) n_{\Omega}^{2} \mathbf{E}_{\Omega} \cdot \mathbf{A}_{\Omega}^{*} d x= \\
& \begin{array}{r}
\int_{\partial \Omega}\left(k^{2}\left(n_{\text {core }}^{2}-n_{\text {clad }}^{2}\right)\left(\mathbf{n} \times \mathbf{E}_{\Omega} \times \mathbf{n}\right) \cdot\left(\mathbf{n} \times \mathbf{A}_{\Omega}^{*} \times \mathbf{n}\right)-k^{2}\left(\frac{1}{n_{\text {core }}^{2}}-\frac{1}{n_{\text {clad }}^{2}}\right)\left(n_{\Omega}^{2} \mathbf{E}_{\Omega} \cdot \mathbf{n}\right)\left(n_{\Omega}^{2} \mathbf{A}_{\Omega}^{*} \cdot \mathbf{n}\right)\right)(\boldsymbol{\theta} \cdot \mathbf{n}) d s \\
+\int_{\mathcal{D}} \boldsymbol{\theta} \cdot r\left(\mathbf{E}_{\Omega}, \mathbf{A}_{\Omega}\right) d x
\end{array}
\end{aligned}
$$

and so, finally,

$$
\begin{aligned}
& J^{\prime}(\Omega)(\boldsymbol{\theta})= \\
& \int_{\partial \Omega} k^{2} \mathcal{R} e\left[\left(n_{\text {clad }}^{2}-n_{\text {core }}^{2}\right)\left(\mathbf{n} \times \mathbf{E}_{\Omega} \times \mathbf{n}\right) \cdot\left(\mathbf{n} \times \mathbf{A}_{\Omega}^{*} \times \mathbf{n}\right)-\left(\frac{1}{n_{\text {clad }}^{2}}-\frac{1}{n_{\text {core }}^{2}}\right)\left(n_{\Omega}^{2} \mathbf{E}_{\Omega} \cdot \mathbf{n}\right)\left(n_{\Omega}^{2} \mathbf{A}_{\Omega}^{*} \cdot \mathbf{n}\right)\right](\boldsymbol{\theta} \cdot \mathbf{n}) d s
\end{aligned}
$$

since the integral on $\mathcal{D}$ must vanish. Hence, the desired result follows.

\section{B Proof of Proposition 4.1}

Let $\delta>0$ be sufficiently small for (Id $+\delta \mathbf{n}_{\Omega}$ ) to be a diffeomorphism from $\mathbb{R}^{3}$ into itself - where, again $\mathbf{n}_{\Omega}$ stands for a smooth extension of the unit normal vector to $\Omega$ to $\mathbb{R}^{3}$. Our purpose is to calculate the shape derivative of the functional

$$
J_{\delta}\left(\Omega_{\boldsymbol{\theta}}\right)=J\left(\left(\mathrm{Id}+\delta \mathbf{n}_{\Omega_{\boldsymbol{\theta}}}\right) \circ(\operatorname{Id}+\boldsymbol{\theta})(\Omega)\right) .
$$


A straightforward calculation yields:

$$
\begin{aligned}
J_{\delta}\left(\Omega_{\boldsymbol{\theta}}\right) & =J\left(\left(\operatorname{Id}+\boldsymbol{\theta}+\delta \mathbf{n}_{\Omega_{\boldsymbol{\theta}}} \circ(\operatorname{Id}+\boldsymbol{\theta})\right)(\Omega)\right), \\
& =J\left(\left(\operatorname{Id}+\delta \mathbf{n}_{\Omega}+\boldsymbol{\theta}+\delta\left(\mathbf{n}_{\Omega_{\boldsymbol{\theta}}} \circ(\operatorname{Id}+\boldsymbol{\theta})-\mathbf{n}_{\Omega}\right)\right)(\Omega)\right), \\
& =J\left(\left(\operatorname{Id}+\boldsymbol{\xi}_{1}(\boldsymbol{\theta})+\delta \boldsymbol{\xi}_{2}(\boldsymbol{\theta})\right) \circ\left(\operatorname{Id}+\delta \mathbf{n}_{\Omega}\right)(\Omega)\right), \\
& =J\left(\left(\operatorname{Id}+\boldsymbol{\xi}_{1}(\boldsymbol{\theta})+\delta \boldsymbol{\xi}_{2}(\boldsymbol{\theta})\right)\left(\Omega_{\delta}\right)\right),
\end{aligned}
$$

where we have defined

$$
\boldsymbol{\xi}_{1}(\boldsymbol{\theta}):=\boldsymbol{\theta} \circ\left(\operatorname{Id}+\delta \mathbf{n}_{\Omega}\right)^{-1} \text {, and } \boldsymbol{\xi}_{2}(\boldsymbol{\theta}):=\left(\mathbf{n}_{\Omega_{\boldsymbol{\theta}}} \circ(\mathrm{Id}+\boldsymbol{\theta})-\mathbf{n}_{\Omega}\right) \circ\left(\mathrm{Id}+\delta \mathbf{n}_{\Omega}\right)^{-1},
$$

considering that $\delta>0$ is small enough so that $\left(\mathrm{Id}+\delta \mathbf{n}_{\Omega}\right)$ is a diffeomorphism. Using the classical formula for the transformation of the normal vector

$$
\mathbf{n}_{\Omega_{\boldsymbol{\theta}}} \circ(\mathrm{Id}+\boldsymbol{\theta})=\frac{1}{\left|\operatorname{com}(\mathrm{I}+\nabla \boldsymbol{\theta}) \mathbf{n}_{\Omega}\right|} \operatorname{com}(\mathrm{I}+\nabla \boldsymbol{\theta}) \mathbf{n}_{\Omega},
$$

whence the "Lagrangian" derivative of the normal vector field $\Omega \mapsto \mathbf{n}_{\Omega}$ is calculated, $\boldsymbol{\xi}_{2}$ expands on $\partial \Omega$ as (see [54] and [18, Chapter 2]):

$$
\boldsymbol{\xi}_{2}(\boldsymbol{\theta}) \circ\left(\mathrm{Id}+\delta \mathbf{n}_{\Omega}\right)=\left(\left(\nabla \boldsymbol{\theta}^{T} \mathbf{n}_{\Omega}\right) \cdot \mathbf{n}_{\Omega}\right) \mathbf{n}_{\Omega}-\nabla \boldsymbol{\theta}^{T} \mathbf{n}_{\Omega}+o(\boldsymbol{\theta}) .
$$

Hence, using the definition (3.7) of the shape derivative of $\Omega \mapsto J(\Omega)$ at $\Omega_{\delta}$, it follows that

$$
J_{\delta}\left(\Omega_{\boldsymbol{\theta}}\right)=J_{\delta}(\Omega)+J^{\prime}\left(\Omega_{\delta}\right)\left(\boldsymbol{\xi}_{1}(\boldsymbol{\theta})+\delta \boldsymbol{\xi}_{2}(\boldsymbol{\theta})\right)+o(\boldsymbol{\theta}),
$$

and we are now left with the calculation of the last quantity in the right-hand side of (B.2); to this end, using Theorem 3.1 yields:

$$
J^{\prime}\left(\Omega_{\delta}\right)\left(\boldsymbol{\xi}_{1}(\boldsymbol{\theta})+\delta \boldsymbol{\xi}_{2}(\boldsymbol{\theta})\right)=\int_{\partial \Omega_{\delta}} g_{\Omega_{\delta}}\left(\boldsymbol{\xi}_{1}(\boldsymbol{\theta})+\delta \boldsymbol{\xi}_{2}(\boldsymbol{\theta})\right) \cdot \mathbf{n}_{\Omega_{\delta}} d s,
$$

where $g_{\Omega}: \partial \Omega \rightarrow \mathbb{R}$ is given by (3.13). Changing variables in the integral in the above right-hand side, we finally obtain:

$$
\begin{aligned}
J^{\prime}\left(\Omega_{\delta}\right)\left(\boldsymbol{\xi}_{1}(\boldsymbol{\theta})+\delta \boldsymbol{\xi}_{2}(\boldsymbol{\theta})\right) & =\int_{\partial \Omega}\left|\operatorname{com}\left(\mathrm{I}+\delta \nabla \mathbf{n}_{\Omega}\right) \mathbf{n}_{\Omega}\right|\left(g_{\Omega_{\delta}}\left(\boldsymbol{\xi}_{1}(\boldsymbol{\theta})+\delta \boldsymbol{\xi}_{2}(\boldsymbol{\theta})\right) \cdot \mathbf{n}_{\Omega_{\delta}}\right) \circ\left(\mathrm{Id}+\delta \mathbf{n}_{\Omega}\right) d s, \\
& =\int_{\partial \Omega} g_{\Omega_{\delta}} \circ\left(\mathrm{Id}+\delta \mathbf{n}_{\Omega}\right) \widetilde{H}\left(\boldsymbol{\theta}, \mathbf{n}_{\Omega}\right) d s+o(\boldsymbol{\theta}) .
\end{aligned}
$$

with

$$
\widetilde{H}\left(\boldsymbol{\theta}, \mathbf{n}_{\Omega}\right):=\left(\boldsymbol{\theta}+\delta\left(\left(\nabla \boldsymbol{\theta}^{T} \mathbf{n}_{\Omega} \cdot \mathbf{n}_{\Omega}\right) \mathbf{n}_{\Omega}-\nabla \boldsymbol{\theta}^{T} \mathbf{n}_{\Omega}\right)\right) \cdot\left(\operatorname{com}\left(\mathrm{I}+\delta \nabla \mathbf{n}_{\Omega}\right) \mathbf{n}_{\Omega}\right) .
$$

where we have used again (B.1). The derivative (B.3) may now be given the convenient structure (3.8) owing to a little calculation. Indeed, let $\boldsymbol{\tau}_{1}, \boldsymbol{\tau}_{2}$ be a local orthonormal basis of the tangent plane of $\partial \Omega$ around a fixed, arbitrary point $\mathbf{x}_{0} \in \partial \Omega$, so that $\left(\boldsymbol{\tau}_{1}(\mathbf{x}), \boldsymbol{\tau}_{2}(\mathbf{x}), \mathbf{n}_{\Omega}(\mathbf{x})\right)$ is an orthonormal basis of $\mathbb{R}^{3}$ for any point $\mathbf{x} \in \partial \Omega$ close to $\mathbf{x}_{0}$. Then the Jacobian matrix of $\mathbf{n}_{\Omega}$ reads in this frame:

$$
\nabla \mathbf{n}_{\Omega}=\left(\begin{array}{ccc}
\kappa_{1} & 0 & 0 \\
0 & \kappa_{2} & 0 \\
0 & 0 & 0
\end{array}\right),
$$

where $\kappa_{i}$ is the principal curvature in direction $\tau_{i}$. Hence,

$$
\begin{aligned}
\left(\left(\nabla \boldsymbol{\theta}^{T} \mathbf{n}_{\Omega}\right) \cdot \mathbf{n}_{\Omega}\right) \mathbf{n}_{\Omega}-\nabla \boldsymbol{\theta}^{T} \mathbf{n}_{\Omega} & =-\sum_{i=1}^{2} \nabla \boldsymbol{\theta}^{T} \mathbf{n}_{\Omega} \cdot \boldsymbol{\tau}_{i} \\
& =-\sum_{i=1}^{2} \nabla\left(\boldsymbol{\theta} \cdot \mathbf{n}_{\Omega}\right) \cdot \boldsymbol{\tau}_{i}+\sum_{i=1}^{2}\left(\nabla \mathbf{n}_{\Omega}^{T} \boldsymbol{\theta}\right) \cdot \boldsymbol{\tau}_{i}, \\
& =-\nabla_{\partial \Omega}\left(\boldsymbol{\theta} \cdot \mathbf{n}_{\Omega}\right)+\sum_{i=1}^{2} \kappa_{i}\left(\boldsymbol{\theta} \cdot \boldsymbol{\tau}_{i}\right) \boldsymbol{\tau}_{i},
\end{aligned}
$$

where $\nabla_{\partial \Omega} f:=\nabla f-\left(\nabla f \cdot \mathbf{n}_{\Omega}\right) \mathbf{n}_{\Omega}$ is the tangential gradient of a smooth enough function $f: \partial \Omega \rightarrow \mathbb{R}$. It follows after a little more algebra that

$$
\widetilde{H}\left(\boldsymbol{\theta}, \mathbf{n}_{\Omega}\right)=\left|\operatorname{det}\left(\operatorname{Id}+\delta \nabla \mathbf{n}_{\Omega}\right)\right|\left(\left(\left(\operatorname{Id}+\delta \nabla \mathbf{n}_{\Omega}\right)^{-1} \mathbf{n}_{\Omega} \cdot \mathbf{n}_{\Omega}\right) \boldsymbol{\theta} \cdot \mathbf{n}_{\Omega}\right),
$$

which is just the factor $H\left(\boldsymbol{\theta}, \mathbf{n}_{\Omega}\right)$ in (4.9); this completes the proof. 


\section{References}

[1] G. Allaire, Conception optimale de structures, vol. 58, Springer, 2007.

[2] G. Allaire and C. Dapogny, A linearized approach to worst-case design in parametric and geometric shape optimization, Mathematical Models and Methods in Applied Sciences, 24 (2014), pp. 2199-2257.

[3] — A deterministic approximation method in shape optimization under random uncertainties, SMAI Journal of Computational Mathematics, 1 (2015), pp. 83-143.

[4] G. Allaire, C. Dapogny, G. Delgado, and G. Michailidis, Multi-phase structural optimization via a level set method, ESAIM: control, optimisation and calculus of variations, 20 (2014), pp. 576-611.

[5] G. Allaire, C. Dapogny, And P. Frey, Shape optimization with a level set based mesh evolution method, Computer Methods in Applied Mechanics and Engineering, 282 (2014), pp. 22-53.

[6] G. Allaire, F. Jouve, And A.-M. Toader, Structural optimization using sensitivity analysis and a level-set method, Journal of computational physics, 194 (2004), pp. 363-393.

[7] S. Amstutz, Aspects théoriques et numériques en optimisation de forme topologique, PhD thesis, 2003.

[8] S. Amstutz and M. Ciligot-Travain, A notion of compliance robustness in topology optimization, ESAIM: Control, Optimisation and Calculus of Variations, 22 (2016), pp. 64-87.

[9] M. P. Bendsoe And O. Sigmund, Topology optimization: theory, methods, and applications, Springer Science \& Business Media, 2013.

[10] J.-P. Berenger, A perfectly matched layer for the absorption of electromagnetic waves, Journal of computational physics, 114 (1994), pp. 185-200.

[11] P. I. Borel, L. H. Frandsen, A. Harpøth, M. Kristensen, J. S. Jensen, and O. Sigmund, Topology optimised broadband photonic crystal y-splitter, Electronics Letters, 41 (2005), pp. 69-71.

[12] M. Burger, A framework for the construction of level set methods for shape optimization and reconstruction, Interfaces and Free boundaries, 5 (2003), pp. 301-329.

[13] C.-L. Chen, Foundations for guided-wave optics, John Wiley \& Sons, 2006.

[14] S. Chen And W. Chen, A new level-set based approach to shape and topology optimization under geometric uncertainty, Structural and Multidisciplinary Optimization, 44 (2011), pp. 1-18.

[15] A. Cherkaev and E. Cherkaeva, Optimal design for uncertain loading condition, in Homogenization: In Memory of Serguei Kozlov, World Scientific, 1999, pp. 193-213.

[16] D. L. Chopp, Computing minimal surfaces via level set curvature flow, Journal of Computational Physics, 106 (1993), pp. 77-91.

[17] COMSOL, Inc., Comsol multiphysics reference manual, version 5.3a.

[18] C. Dapogny, Shape optimization, level set methods on unstructured meshes and mesh evolution, PhD thesis, Université Pierre et Marie Curie-Paris 6, 2013.

[19] C. Dapogny, R. Estevez, A. Faure, And G. Michailidis, Shape and topology optimization considering anisotropic features induced by additive manufacturing processes, Hal preprint: https://hal.archivesouvertes.fr/hal-01660850/, (2017).

[20] R. Dautray and J.-L. Lions, Mathematical analysis and numerical methods for science and technology: volume 3 Spectral Theory and Applications, Springer Science \& Business Media, 2012.

[21] F. De Gournay, Velocity extension for the level-set method and multiple eigenvalues in shape optimization, SIAM journal on control and optimization, 45 (2006), pp. 343-367.

[22] M. C. Delfour And J.-P. ZolÂsio, Shapes and geometries: metrics, analysis, differential calculus, and optimization, vol. 22, Siam, 2011. 
[23] A.-S. B.-B. Dhia AND P. Joly, Mathematical analysis and numerical approximation of optical waveguides, in Mathematical modeling in optical science, SIAM, 2001, pp. 273-324.

[24] Y. Elesin, B. S. Lazarov, J. S. Jensen, and O. Sigmund, Design of robust and efficient photonic switches using topology optimization, Photonics and nanostructures-Fundamentals and Applications, 10 (2012), pp. 153-165.

[25] — Time domain topology optimization of 3d nanophotonic devices, Photonics and NanostructuresFundamentals and Applications, 12 (2014), pp. 23-33.

[26] A. Farjadpour, D. Roundy, A. Rodriguez, M. Ibanescu, P. Bermel, J. Joannopoulos, S. G. JoHnSON, AND G. BURR, Improving accuracy by subpixel smoothing in the finite-difference time domain, Optics letters, 31 (2006), pp. 2972-2974.

[27] S. Fathpour and B. Jalali, Silicon photonics for telecommunications and biomedicine, CRC Press, 2011.

[28] F. Feppon, G. Allaire, F. Bordeu, J. Cortial, and C. Dapogny, Shape optimization of a coupled thermal fluid-structure problem in a level set mesh evolution framework, submitted, (2018).

[29] L. F. Frellsen, Y. Ding, O. Sigmund, And L. H. Frandsen, Topology optimized mode multiplexing in silicon-on-insulator photonic wire waveguides, Optics express, 24 (2016), pp. 16866-16873.

[30] A. Friedman, Mathematical problems in electron beam lithography, in Mathematics in Industrial Problems, Springer, 1989, pp. 79-87.

[31] B. Goursaud, Etude mathématique et numérique de guides d'ondes ouverts non uniformes, par approche modale, PhD thesis, Ecole Polytechnique X, 2010.

[32] D. J. Griffiths, Introduction to electrodynamics, 2005.

[33] X. Guo, W. Zhang, and L. Zhang, Robust structural topology optimization considering boundary uncertainties, Computer Methods in Applied Mechanics and Engineering, 253 (2013), pp. 356-368.

[34] J. Hadamard, Mémoire sur le problème d'analyse relatif à l'équilibre des plaques élastiques encastrées, vol. 33, Imprimerie nationale, 1908.

[35] A. Henrot and M. Pierre, Variation et optimisation de formes: une analyse géométrique, vol. 48, Springer Science \& Business Media, 2006.

[36] M. Hintermüller, A. Laurain, And I. Yousept, Shape sensitivities for an inverse problem in magnetic induction tomography based on the eddy current model, Inverse Problems, 31 (2015), p. 065006.

[37] R. G. Hunsperger, Integrated optics, vol. 4, Springer, 1995.

[38] H. Jansen, H. Gardeniers, M. De Boer, M. Elwenspoek, and J. Fluitman, A survey on the reactive ion etching of silicon in microtechnology, Journal of micromechanics and microengineering, 6 (1996), p. 14.

[39] J. S. Jensen and O. Sigmund, Topology optimization for nano-photonics, Laser \& Photonics Reviews, 5 (2011), pp. 308-321.

[40] J.-M. Jin, The finite element method in electromagnetics, John Wiley \& Sons, 2014.

[41] S. G. Johnson, M. Ibanescu, M. Skorobogatiy, O. Weisberg, J. Joannopoulos, and Y. Fink, Perturbation theory for maxwell's equations with shifting material boundaries, Physical review E, 65 (2002), p. 066611.

[42] O. Kononchuk And B.-Y. NGuyen, Silicon-on-insulator (soi) technology: Manufacture and applications, Elsevier, 2014.

[43] C. M. Lalau-Keraly, S. Bhargava, O. D. Miller, and E. Yablonovitch, Adjoint shape optimization applied to electromagnetic design, Optics Express, 21 (2013), p. 21693. 
[44] B. S. Lazarov, M. Schevenels, And O. Sigmund, Topology optimization with geometric uncertainties by perturbation techniques, International Journal for Numerical Methods in Engineering, 90 (2012), pp. 1321-1336.

[45] J. Lu And J. VuČKović, Nanophotonic computational design, Optics express, 21 (2013), pp. 1335113367.

[46] P. MADER, Optimisation topologique en propagation électromagnétique guidée, $\mathrm{PhD}$ thesis, Université Paul Sabatier, 2002.

[47] J. Martínez-Frutos, D. Herrero-Pérez, M. Kessler, and F. Periago, Robust shape optimization of continuous structures via the level set method, Computer Methods in Applied Mechanics and Engineering, 305 (2016), pp. 271-291.

[48] K. Maute, Topology optimization under uncertainty, in Topology optimization in structural and continuum mechanics, Springer, 2014, pp. 457-471.

[49] H. Men, K. Y. Lee, R. M. Freund, J. Peraire, and S. G. Johnson, Robust topology optimization of three-dimensional photonic-crystal band-gap structures, Optics express, 22 (2014), pp. 22632-22648.

[50] G. Michailidis, Manufacturing constraints and multi-phase shape and topology optimization via a levelset method, PhD thesis, Ecole Polytechnique X, 2014.

[51] I. M. Mitchell, The flexible, extensible and efficient toolbox of level set methods, Journal of Scientific Computing, 35 (2008), pp. 300-329.

[52] S. Molesky, Z. Lin, A. Y. Piggott, W. Jin, J. Vucković, And A. W. Rodriguez, Inverse design in nanophotonics, Nature Photonics, 12 (2018), p. 659.

[53] P. Monk, Finite element methods for Maxwell's equations, Oxford University Press, 2003.

[54] F. Murat and J. Simon, Sur le contrôle par un domaine géométrique, Preprint,(76015), (1976), pp. $725-734$.

[55] S. Osher And J. A. Sethian, Fronts propagating with curvature-dependent speed: algorithms based on hamilton-jacobi formulations, Journal of computational physics, 79 (1988), pp. 12-49.

[56] J. Отт, Halfspace Matching: a Domain Decomposition Method for Scattering by 2D Open Waveguides, $\mathrm{PhD}$ thesis, Karlsruher Instituts für Technologie, 2017.

[57] O. Pantz, Sensibilité de l'équation de la chaleur aux sauts de conductivité, Comptes Rendus Mathematique, 341 (2005), pp. 333-337.

[58] A. Y. Piggott, J. Lu, K. G. Lagoudakis, J. Petykiewicz, T. M. Babinec, and J. Vučković, Inverse design and demonstration of a compact and broadband on-chip wavelength demultiplexer, Nature Photonics, 9 (2015), pp. 374-377.

[59] A. Y. Piggott, J. Petykiewicz, L. Su, And J. VuČKović, Fabrication-constrained nanophotonic inverse design, Scientific Reports, 7 (2017), p. 1786.

[60] B. SAmet, The topological asymptotic analysis for the Maxwell equations and applications, $\mathrm{PhD}$ thesis, Université Paul Sabatier-Toulouse III, 2004.

[61] S. Sargheini, A. Paganini, R. Hiptmair, and C. Hafner, Shape sensitivity analysis of metallic nano particles, International Journal of Numerical Modelling: Electronic Networks, Devices and Fields, 30 (2017).

[62] J. A. Sethian, Level set methods and fast marching methods: evolving interfaces in computational geometry, fluid mechanics, computer vision, and materials science, vol. 3, Cambridge university press, 1999.

[63] J. A. Sethian And A. Wiegmann, Structural boundary design via level set and immersed interface methods, Journal of computational physics, 163 (2000), pp. 489-528. 
[64] B. Shen, P. Wang, R. Polson, And R. Menon, An integrated-nanophotonics polarization beamsplitter with 2.4× $2.4 \mu \mathrm{m} 2$ footprint, Nature Photonics, 9 (2015), p. 378.

[65] O. Sigmund, Manufacturing tolerant topology optimization, Acta Mechanica Sinica, 25 (2009), pp. 227239.

[66] A. W. Snyder And J. Love, Optical waveguide theory, Springer Science \& Business Media, 2012.

[67] L. Su, R. Trivedi, N. V. Sapra, A. Y. Piggott, D. Vercruysse, and J. Vučković, Fullyautomated optimization of grating couplers, Optics express, 26 (2018), pp. 4023-4034.

[68] The MathWorks, Inc., Matlab, version 2018a.

[69] O. Tsilipakos, E. Kriezis, and T. Yioultsis, Boundary condition for the efficient excitation and absorption of hybrid waveguide modes in finite element formulations, Microwave and Optical Technology Letters, 53 (2011), pp. 2626-2631.

[70] F. Wang, J. S. Jensen, And O. Sigmund, Robust topology optimization of photonic crystal waveguides with tailored dispersion properties, JOSA B, 28 (2011), pp. 387-397.

[71] M. Y. Wang, X. Wang, And D. Guo, A level set method for structural topology optimization, Computer methods in applied mechanics and engineering, 192 (2003), pp. 227-246.

[72] Z. Yu, H. CuI, AND X. Sun, Genetic-algorithm-optimized wideband on-chip polarization rotator with an ultrasmall footprint, Optics letters, 42 (2017), pp. 3093-3096.

[73] Y. Zhu AND A. C. CANGellaris, Multigrid finite element methods for electromagnetic field modeling, vol. 28, John Wiley \& Sons, 2006. 